Review

\title{
Structural Aspects of the Bechgaard and Fabre Salts: An Update
}

\section{Jean-Paul Pouget}

Laboratoire de Physique des Solides, Université Paris-sud, CNRS, UMR 8502, Bâtiment 510, Orsay Cedex F-91405, France; E-Mail: pouget@lps.u-psud.fr; Tel.: +33-169-156-046;

Fax: +33-169-156-936

Received: 20 March 2012; in revised form: 19 April 2012 / Accepted: 20 April 2012/

Published: 21 May 2012

\begin{abstract}
We review structural aspects of the Bechgaard and Fabre salts in relationship with their electronic, magnetic and superconducting properties. We emphasize the role of bond and charge modulations of the quarter filled organic stack in the various instabilities and ground states exhibited by these salts. A special consideration is also devoted to the influence of anions and methyl groups in these processes. In particular we point out the importance of the anions in achieving the inter-stack coupling by either direct or indirect (via the polarization of the methyl group cavities) interactions with the donors. In this framework we discuss the role of anions and methyl group disorders in the inhibition of the divergence of the high temperature bond order wave instability of the Bechgaard salts. We analyze the modulation in the magnetic ground states by considering explicitly the coupling of the magnetization with structural degrees of freedom. We consider the role of the anions and methyl groups in stabilizing the charge ordering pattern in the Fabre salts. We also discuss the spin-Peierls transition of the Fabre salts in relation with the charge ordering transition and the adiabaticity of the phonon field. We review the anion ordering transitions by considering more particularly the influence of the ordering process on the electronic structure and on the ground states which results. In this framework we show that the texture of the anion ordered structure has direct consequences on the superconducting properties of $(\mathrm{TMTSF})_{2} \mathrm{ClO}_{4}$. Finally we conclude on the essential implication of the structural degrees of freedom on the generic phase diagram of the Bechgaard and Fabre salts.
\end{abstract}


Keywords: one-dimensional metals; strongly correlated fermions; organic conductors; organic superconductors; charge order; spin-Peierls transitions; anion ordering transitions; charge density wave; spin density wave; X-ray diffuse scattering

\section{Introduction}

For several decades the synthesis of new classes of materials has triggered original fields of research where the discovery of new electronic states of matter has increasingly enriched the number of fundamental concepts in solid state physics while opening new routes for potential applications. This has been the case, to name a few examples, of oxides and carbon based compounds including conducting polymers. In this respect, the study of organic conductors since the beginning of the 1970s has largely contributed to this revival [1-3].

Organic conductors presenting a regular array of quasi-planar organic molecules, developing a preferential overlap of their $\mathrm{p}_{\pi} \mathrm{HOMO}$ in one or two crystallographic directions, provide very anisotropic materials exhibiting a relatively simple electronic structure. The originality of these materials relies on the following facts:

(i) Their $\mathrm{p}_{\pi}$ electron bandwidth is comparable to the electron-electron Coulomb repulsions which, being weakly screened, decrease slowly with the intermolecular separation.

(ii) Their lattice is soft and quite compressible, which leads to rapid change of the electronic properties under pressure or uniaxial stress, while providing a sizeable coupling between the electronic and structural degrees of freedom.

(iii) Their crystal array is quite perfect, which allows a fine control of the perturbation of physical properties by defects intentionally introduced in the structure.

If one restricts to quasi one-dimensional (1D) systems, the organic conductors provide classes of materials which realize a very large panel of different physical situations. The charge transfer salts of the TTF-TCNQ family are composed of segregated stacks of donors (D: TTF) and acceptors (A: TCNQ) with generally an incommensurate charge transfer between the two metallic 1D subsystems. These salts are subject to a charge density wave (CDW) instability which triggers a Peierls insulating ground state [1]. The 2:1 $\mathrm{A}_{2} \mathrm{Y}$ or $\mathrm{D}_{2} \mathrm{X}$ salts, where $\mathrm{Y}$ or $\mathrm{X}$ is a monovalent cationic or anionic entity, form quasi-1D quarter filled electronic systems built exclusively on acceptors, A, or donors, D. These "less screened" metallic systems generally experience quite strong Coulomb repulsions driving high temperature charge localization. This localization, accompanied by a spin charge decoupling, leaves the spin degrees of freedom active for a low temperature anti-ferromagnetic (AF) or spin-Peierls (SP) instability. Among these 2:1 series considerable studies have been devoted to the so-called Fabre and Bechgaard salts built with TMTTF and TMTSF donors respectively. The Fabre salts show charge localization, spin-charge decoupling and prototypal examples of AF and SP ground states while the Bechgaard salts exhibit a Fermi surface (FS) nesting driven spin density wave (SDW) ground state. The suppression of the SDW ground state under pressure restores the metallic state which is thus subject to superconductivity [1,2]. To complete this survey of different classes of organic 1D system, let us briefly mention very original 2:1 series of quasi-1D organic systems based on acceptors 
incorporating a metal transition element $\mathrm{M}$. The corresponding salts, in presence of perylene or of TTF based donor, achieve segregated metallic and magnetic stacks [ $\mathrm{Per}_{2}-\mathrm{M}(\mathrm{mnt})_{2}$ series [4]] or spin ladders [(DT-TTF $)_{2}-\mathrm{M}(\mathrm{mnt})_{2}$ series [5]]. Finally, let us mention conducting and even superconducting 2:1 $\left(\mathrm{M}(\mathrm{dmit})_{2}\right)_{2} \mathrm{Y}$ or charge transfer $\operatorname{TTF}\left(\mathrm{M}(\mathrm{dmit})_{2}\right)_{2}$ systems [2], where the electronic structure of the $\mathrm{M}(\mathrm{dmit})_{2}$ acceptor, composed of dmit side groups weakly coupled through a central $\mathrm{M}$ atom, involves both HOMO and LUMO electronic states [6]. These highly original conducting salts, which are not considered here, deserve a special review in their own right.

This present review focuses on structural aspects of Bechgaard and Fabre salts and their relationship with electronic, magnetic and superconducting properties. It will cover the most recent findings concerning the structural instabilities exhibited by these salts, and will present quantitative analyses of earlier data. In this respect this paper updates our 15 year old reviews [7,8]. The review is organized as follows: The structure of the Bechgaard and Fabre salts is presented in Section 2 with special consideration of entities achieving a subtle coupling to the electronic degrees of freedom. The underlying bond order wave (BOW) instability, always present in these salts, is quantitatively analyzed in Section 3. The AF and SDW magnetic ground states are described in Section 4, which also includes the often ignored coupling between magnetic and structural order parameters. Section 5 considers charge ordering (CO) effects in the Fabre salts with a special investigation of the role of the anions and methyl groups in the stabilization of the CO ground state. Section 6 is devoted to the SP transition and its coupling with the CO. Section 7 will review the most recent findings concerning the influence of the anion ordering (AO) transitions on the electronic structures. Finally we conclude this review by summarizing the influence of the structural degrees of freedom on the generic phase diagram of the Fabre and Bechgaard salts.

\section{Basic Aspects of the Crystallographic Structure in Relationship with Electronic Properties}

\subsection{Structural Arrangement}

As shown in Figure 1, Bechgaard and Fabre salts are made of a zig-zag stacking of TMTSF and TMTTF molecules arranged nearly perpendicularly to the 1D direction, a. In the short direction of the molecule, stacks, shifted from each other along b, form (a, b) donor layers. In the long direction of the molecule, out of phase first neighboring zig-zag stacks delimit methyl group cavities which are filled by the anions $\mathrm{X}$. This packing leads to an alternation of layers of well coupled donors with anions along c. Two aspects of the structure at ambient conditions are noticeable: (i) the slight dimerization of the zig-zag stack; and (ii) the presence of a sizeable thermal motion of the anion X in its cavity [9]. These salts crystallize in the triclinic space group P-1 with the inversion centers located in between the molecules and in the center of the methyl group cavities. If one ignores the slight stack dimerization and the location of the anions close to the shortest zig-zag bond, the structure has an underlying monoclinic $\mathrm{C} 2 / \mathrm{m}$ symmetry (a: binary axis, $\mathrm{b}^{\prime}=2 \mathrm{~b}-\mathrm{a}, \mathrm{c}$ ) tending to promote a 2 screw axis symmetry in the stack direction [8]. In this respect it is interesting to remark that both Bechgaard and Fabre salts, with $\mathrm{X}=\mathrm{PF}_{6}$, undergo, at $5.5 \mathrm{GPa}$ and $8.5 \mathrm{GPa}$ respectively, a first order phase transition to a monoclinic (a: binary axis, b', c) structure [10]. In the (a, b) donor layer, this discontinuous structural change could be achieved by the relative shift, along a, of first neighboring stacks. 
Figure 1. Structure of (TMTSF) ${ }_{2} \mathrm{PF}_{6}$ : (a) Projection in the (a, [011]) plane; (b) Projection along a. The $\mathrm{F}$ atoms are labeled. In (b) the methyl groups located in the low temperature neutron scattering structural refinement [11] are shown. The shortest $F(1)$-Se distances, $\mathrm{F}(1)-\mathrm{H}$ and $\mathrm{F}(3)-\mathrm{H}$ bonds are indicated by the blue and red dotted lines respectively.

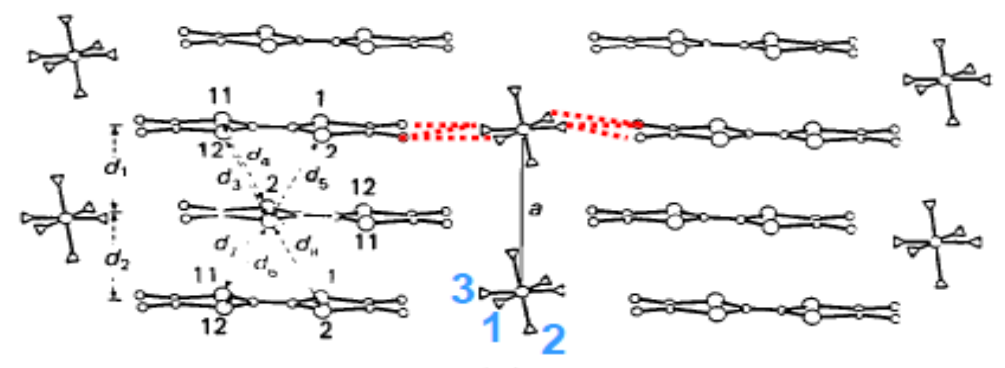

(a)

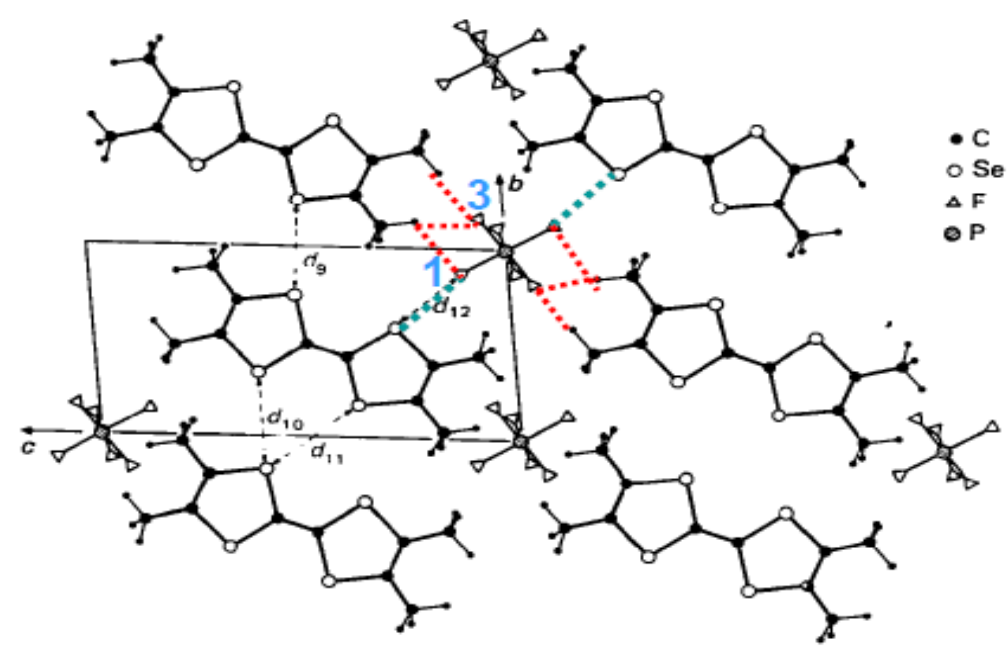

(b)

\subsection{Dimerization of the Stack}

In a $1 \mathrm{D}$ description of the quarter filled interacting electron gas, the dimerization of the zig-zag stack activates an umklapp $4 \mathrm{k}_{\mathrm{F}}=\mathrm{a}^{*}$ electron-electron scattering term which, in presence of sizeable Coulomb repulsions, facilitates the charge localization $\left(\mathrm{k}_{\mathrm{F}}\right.$ is the Fermi wave vector of the quarter filled $1 \mathrm{D}$ electron gas) [12]. The strength of this umklapp process depends of the amplitude of the dimerization gap $2 \Delta_{\mathrm{D}}$ which opens at $\pm \mathrm{a} * / 2$ in the band structure well below the Fermi level. $\Delta_{\mathrm{D}}$ amounts to the difference of transfer integrals (in presence of the Hartree anion potential) on the two inequivalent bonds of the zig-zag. $\Delta_{\mathrm{D}}$ calculated by the DFT differs from $\Delta_{\mathrm{D}}$ calculated by the Extended Hückel Theory (EHT) which does not take into account the anion potential. For example in (TMTSF) ${ }_{2} \mathrm{ClO}_{4}$ at room temperature (RT) DFT gives $\Delta_{\mathrm{D}}=26 \mathrm{meV}$ [13], while EHT gives $\Delta_{\mathrm{D}}=37-54 \mathrm{meV}$ (see Table 4.3 in [2]). Another complication arises if one considers the interstack coupling: the inter-chain transfer integrals lead to a significant variation of $\Delta_{\mathrm{D}}$ along $\mathrm{b}^{*}$. In (TMTSF) $)_{2} \mathrm{ClO}_{4} \Delta_{\mathrm{D}}$ varies from $\sim 50 \mathrm{meV}$ in $\left(\mathrm{a}^{*} / 2,0\right)$ to $\sim 0 \mathrm{meV}$ in $\left(\mathrm{a}^{*} / 2, \mathrm{~b} * / 2\right)$ [13] at RT. $\Delta_{\mathrm{D}}$ decreases upon cooling and under pressure, while $\Delta_{\mathrm{D}}$ increases from the TMTSF'salts to the TMTTF'salts. $\Delta_{\mathrm{D}}$ is controlled by the shape and volume of the anion which deforms the donor packing via its interaction with the terminal methyl groups. 


\subsection{Anion Cavities}

Structural effects associated to anions require a special attention because the anions are located in soft cavities delimited by the methyl groups of the TMTSF or the TMTTF molecules. The anions fit these cavities in a more or less compact manner depending upon the volume and shape of the anion [14-16]. The anions were considered in earlier studies as spacers acting on the inter-layer distance as does the pressure [17]. In fact recent works summarized in this review show that their role is more subtle than it was previously believed. First, the anions are located in centro-symmetric cavities. This location causes no problem for centro-symmetric anions such as octahedron $\mathrm{MF}_{6}(\mathrm{M}=\mathrm{P}, \mathrm{As}, \mathrm{Sb})$ or sphere $(\mathrm{Br})$, but it implies at RT the existence of an orientation disorder for tetrahedral $\left(\mathrm{ReO}_{4}, \mathrm{ClO}_{4}, \mathrm{BF}_{4}\right)$, triangular $\left(\mathrm{NO}_{3}\right)$ or asymmetric linear (SCN) anions. Second, each anion experiences quite a symmetric environment from its six first neighbor donors [18] due to the six closest methyl groups. Among them, four groups are located in the (b', $c^{*}$ ) plane perpendicular to the stacking axis, while the two remaining groups, located in the $\left(\mathrm{a}, \mathrm{c}^{*}\right)$ plane, are significantly tilted with respect to a. These six methyl groups delimit three sets of three-fold symmetry axis close to the symmetry axis of the octahedral anions. The four planar methyl groups delimit two sets of two-fold symmetry axis close to the symmetry axis of the tetrahedral anions [18]. Third, the $\mathrm{F}$ or $\mathrm{O}$ outer atoms of the anion form weak H-bonds with the closest methyl groups, especially at low temperatures when thermal disorder is reduced [19]. The $4 \mathrm{~K}$ neutron scattering structural refinement [11], which allows precisely locating the $\mathrm{H}$ of the methyl groups of (TMTSF) $)_{2} \mathrm{PF}_{6}$, shows the formation of $6 \mathrm{H}$-bonds with $\mathrm{F}(1)$ and $\mathrm{F}(3)$ of the $\mathrm{PF}_{6}$ (red dotted lines in Figure 1). All these $\mathrm{H}$-bonds are located in the plane perpendicular to the stacking axis a. In addition, the anion develops short contact distances with the Se of the closest donors. Figure 1 shows (blue dotted lines) the two short $\mathrm{Se} . . \mathrm{F}(1)$ contacts established in (TMTSF) ${ }_{2} \mathrm{PF}_{6}$. The anion and thermal dependences of the geometry of the $\mathrm{S}$...F interactions have been analyzed in (TMTTF) ${ }_{2} \mathrm{PF}_{6}, \mathrm{AsF}_{6}$ and $\mathrm{SbF}_{6}$ [20]. Note that $\mathrm{F}(2)$, which presents the strongest disorder [11], is not involved in any interactions. Any change of the shape of the methyl group cavity, caused by an elastic deformation of the donor stack for example, should react on the location of the anions and vice versa.

These two kinds of short contacts provide respectively indirect (via the polarization of the H-bond network and of the intra-molecular $\sigma$ bonds) [21] and direct (via the Se or S atoms) attractive interactions between the anion and the $\pi$ holes cloud located in the inner part of the donor. In this respect infrared measurements provided evidence of a considerable electron-molecular-vibration coupling of the methyl groups of both TMTSF and TMTTF with the charges on the molecules [22]. These interactions are involved in the inter-chain coupling mechanism (see Section 3.1.2) and for this reason play a key role in the stabilization of various ground states exhibited by Bechgaard and Fabre salts such as the AO transition involving non centro-symmetric anions (see Section 7), the CO transition breaking the inversion symmetry (see Section 5) and the SP transition subject to an important magneto-elastic coupling (see Section 6).

\subsection{Anion and Methyl Group Disorders}

The location of anions in methyl group cavities provide flexibility in the structure due to the soft interface provided by the methyl groups between the anions and the core of the donors and the 
incomplete fit of the anion in the volume delimited by these methyl groups [14-16]. This soft environment tolerates the presence of disordered anions in the structure even for centro-symmetrical anions [9]. At ambient conditions, the non centro-symmetrical tetrahedral anions are disordered in Bechgaard and Fabre salts while they are ordered in others structurally related 2:1 salts, such as (BEDT-TTF $)_{2} \mathrm{ReO}_{4},(\mathrm{t}-\mathrm{TTF})_{2} \mathrm{X}$ with $\mathrm{X}=\mathrm{ClO}_{4}$ and $\mathrm{BF}_{4}$ and $(\mathrm{DMtTTF})_{2} \mathrm{ClO}_{4}$, built with donors which do not provide a methyl group environment at the anion.

The lattice softness is revealed by thermal expansion measurements. First, linear expansion coefficients, $\alpha_{i}$, are one order of magnitude larger in the $\mathrm{PF}_{6}$ salt than in the $\mathrm{Br}$ salt where the anion better fits the volume of its methyl group cavity [23]. Second, in salts incorporating $\mathrm{PF}_{6}$ or $\mathrm{AsF}_{6}$ anions, the rate of variation of $\alpha_{\mathrm{i}}$ upon heating becomes negative at high temperature [24]. This finding points out peculiar lattice dynamics, possibly due to "free" rotation and/or translation of the anions in their cavities. This effect, which is observed both for TMTTF and TMTSF salts, is mostly pronounced along the $\mathrm{c}^{*}$ direction where layers of donors and anions alternate [23-25].

The enhanced values of Debye Waller factors obtained in structural refinements show the presence of an atomic disorder for both the methyl groups and the outer atoms of the anions. An orientation disorder is expected for non centro-symmetrical anions located in a centro-symmetric cavity. This disorder is removed at a well defined AO transition which will be the object of Section 7. Below we shall consider the case of centro-symmetrical octahedral anions such as $\mathrm{PF}_{6}$. At RT, F atoms exhibit a sizeable thermal motion but the finding of well defined maxima in electron-density map, obtained in the structural refinement of $(\mathrm{TMTSF})_{2} \mathrm{PF}_{6}$ [9], show that there is no free rotation of the centrosymmetrical anion. At $20 \mathrm{~K}$, the anion disorder is almost completely suppressed especially for $\mathrm{F}(1)$ and $\mathrm{F}(3)$ atoms forming $\mathrm{H}$ bonds and a linkage with the Se (see Figure 1) [11]. However neutron refinements show that methyl groups sustain a certain disorder even at $4 \mathrm{~K}$ [11], probably due to quantum-mechanical tunneling. NMR studies show that $\mathrm{PF}_{6}$ disorder is established upon heating above $\sim 70 \mathrm{~K}$ in $(\mathrm{TMTSF})_{2} \mathrm{PF}_{6}$ [26]. More recent NMR studies performed in (TMTTF) ${ }_{2} \mathrm{SbF}_{6}[27,28]$ show, by the measurement of different activation energies in the molecular motion, that in fact anion disorder is progressively set upon heating. A detailed analysis [29] of the Debye Waller factor of (TMTSF) ${ }_{2} \mathrm{AsF}_{6}$ at $125 \mathrm{~K}$ suggests that the disorder could take place between two well defined orientations of the AsF $_{6}$ anion in its cavity. Several activation energies also govern the motion of the methyl groups in TMTSF salts [30-33].

Thermal variation of the principal directions of the dilatation tensor of $(\mathrm{TMTSF})_{2} \mathrm{PF}_{6}$ [11] suggests a lattice modification around $60-50 \mathrm{~K}$ which could be related to the "freezing out" of the $\mathrm{PF}_{6}$ disorder previously considered. More precisely, the thermal variation of the lattice expansion in the direction $i$ divided by $T, \alpha_{\mathrm{i}} / \mathrm{T}$, or more likely the volume expansion divided by $T,\left(\Sigma_{\mathrm{i}} \alpha_{\mathrm{i}}\right) / \mathrm{T}$, is proportional to the entropy derivative $\partial \mathrm{S} / \partial \mathrm{T}$ [34]. $\alpha \perp / \mathrm{T}$ taken from the data of [24] exhibits broad maxima around 30-40 $\mathrm{K}$ in (TMTSF) ${ }_{2} \mathrm{PF}_{6}$ before dropping to zero at lower temperature [34]. This finding implies that below these maxima a net decrease of the "lattice" entropy, probably due to the vanishing of the structural disorder, should occur. The best way to reduce simultaneously the $\mathrm{PF}_{6}$ and methyl group disorders is to link these two entities, as suggested from methyl protons NMR [32]. These findings have important consequences on the density wave instabilities which will be considered in Section 3. 
The $\mathrm{PF}_{6}$ anion disorder could be even more significantly reduced by a well defined thermodynamic transformation recalling the AO transition (see Section 7). This could be achieved by an orientation ordering transition achieving a well defined orientation at the anion which oscillates between several positions in its methyl group cavity at high temperature [29]. Such a transition has been observed in (EDO-TTF $)_{2} \mathrm{PF}_{6}$ [35] where the orientation ordering of $\mathrm{PF}_{6}$, accompanied by its shift, stabilizes a 3D pattern of $\mathrm{CO}$ and BOW distortions of the EDO-TTF stack. All these effects lead to a unit cell doubling which causes the $2 \mathrm{k}_{\mathrm{F}}$ MI transition of (EDO-TTF) ${ }_{2} \mathrm{PF}_{6}$. In P-1 triclinic (TMTSF) $)_{2} \mathrm{PF}_{6}$, where there is no cell doubling (above $T_{\mathrm{SDW}}$ ), the only possible symmetry breaking operation while keeping the translation symmetry will be the loss of inversion centers as for the $\mathrm{CO}$ transition of the $(\text { TMTTF })_{2}$ X's (see Section 5). However neutron scattering structural refinements of (TMTSF) ${ }_{2} \mathrm{PF}_{6}$ performed at $4 \mathrm{~K}$ and $20 \mathrm{~K}$ [11] do not provide any evidence of the loss of the inversion centers (Note that this also implies that $\mathrm{PF}_{6}$ does not shift from the centre of its methyl group cavity).

\section{The Bond Order Wave Instability in the Bechgaard and Fabre Salts}

\subsection{The Interplay between $2 k_{F} B O W$ and $S D W$ Instabilities in $(T M T S F)_{2} \mathrm{PF}_{6}$}

\subsubsection{Quantitative Analysis of the 1D $2 \mathrm{k}_{\mathrm{F}}$ BOW Instability}

All charge transfer salts of the TTF-TCNQ family exhibit a divergent $2 \mathrm{k}_{\mathrm{F}}$ CDW instability which, when coupled to the lattice degrees of freedom, drives a low temperature $T_{\mathrm{P}}(\sim 30-50 \mathrm{~K})$ metal-insulator (MI) transition [1,36]. In TTF-TCNQ the electron-phonon coupling of the 1D electron gas with the lattice modes leads to the formation above $T_{\mathrm{P}}$ of a Kohn anomaly in a transverse acoustic (TA) branch [37] which softening at $T_{\mathrm{P}}$ leads to a static modulation of the intra-stack bond distances. This stabilizes a $2 \mathrm{k}_{\mathrm{F}}$ BOW ground state below $T_{\mathrm{P}}$. Such instability of a $1 \mathrm{D}$ metal with respect to a $2 \mathrm{k}_{\mathrm{F}}$ periodic lattice distortion (PLD) was first proposed by Peierls in 1955.

Above $T_{\mathrm{P}}$ the 1D BOW structural instability gives also rise to critical fluctuations consisting, in reciprocal space, in a planar X-ray diffuse scattering (or diffuse lines on X-ray patterns) at the wave vector $\mathrm{Q}=\mathrm{G}+\mathrm{q}$, where $\mathrm{G}$ is a reciprocal lattice wave vector and $\mathrm{q} \sim 2 \mathrm{k}_{\mathrm{F}}$. The intensity, $\mathrm{I}(\mathrm{Q})$, of this diffuse scattering can be expressed in the form [36,38]:

$$
\mathrm{I}(\mathrm{Q})=|\mathrm{F}(\mathrm{Q})|^{2}\left\langle\left|\mathrm{u}_{\mathrm{q}}\right|^{2}\right\rangle
$$

In expression (1) $\mathrm{u}_{\mathrm{q}}$ is the atomic displacement (of the TA critical phonon mode at $\mathrm{q}$ in TTF-TCNQ for example) and $F(Q)$ is the structure factor of the modulation. In the classical limit when the thermal energy $k_{B} T$ is larger than the critical phonon energy, $\hbar \Omega$, one has:

$$
<\left|\mathrm{u}_{\mathrm{q}}\right|^{2}>\approx k_{B} T \chi(\mathrm{q})
$$

In expression (2) $\chi(\mathrm{q})$ is the CDW or BOW response function of the electron-phonon coupled system. For a standard Peierls chain $\chi(\mathrm{q})$ is peaked at the $2 \mathrm{k}_{\mathrm{F}}$ wave vector. In the regime of $1 \mathrm{D}$ fluctuations $\chi(\mathrm{q})$ has a Lorentzian dependence in q:

$$
\chi(\mathrm{q})=\chi\left(2 \mathrm{k}_{\mathrm{F}}\right) /\left[1+\xi^{2}\left(\mathrm{q}-2 k_{\mathrm{F}}\right)^{2}\right]
$$


In expression (3) the correlation length of the intra-chain structural fluctuations, $\xi$, is given by the inverse half width at half maximum (HWHM) of $\chi(\mathrm{q})$. In the case of a Peierls instability (case of TMTSF-DMTCNQ considered below in Figure 2$)$ both $\chi\left(2 k_{\mathrm{F}}\right)$ and $\xi$ diverge at $T_{\mathrm{P}}$.

The surprise of the investigation of the Bechgaard salts was the observation in (TMTSF) ${ }_{2} \mathrm{AsF}_{6}$ [39] and $\mathrm{PF}_{6}$ [40] of $\mathrm{X}$-ray diffuse lines, corresponding to the $1 \mathrm{D} 2 \mathrm{k}_{\mathrm{F}}$ BOW instability previously described, whose intensity $\mathrm{I}(\mathrm{Q})$ progressively vanishes upon cooling below $\sim 50 \mathrm{~K}$. Below $\sim 30 \mathrm{~K}$ these structural fluctuations are no longer observable. The MI transition, which occurs at $T_{\mathrm{MI}}=12 \mathrm{~K}$ in these salts, is thus not due to the setting of a Peierls ground state. The MI is caused by the establishment of a $2 \mathrm{k}_{\mathrm{F}}$ SDW order [1,2] by a mechanism similar to the one proposed by Slater in 1951 [41]. In this mechanism the divergence of $2 \mathrm{k}_{\mathrm{F}}$ SDW (AF in the model of Slater) correlations give rise to a $2 \mathrm{k}_{\mathrm{F}}$ periodic exchange potential which opens a gap at the Fermi level in the 1D band structure and thus drives the electronic system to an insulating ground state (here the $2 \mathrm{k}_{\mathrm{F}}$ periodic exchange potential plays the role of the $2 \mathrm{k}_{\mathrm{F}}$ PLD of the Peierls transition).

The reason for the low temperature vanishing of the $2 \mathrm{k}_{\mathrm{F}}$ BOW instability in the Bechgaard salts has never been elucidated. To this end, we compare below the $2 \mathrm{k}_{\mathrm{F}} \mathrm{BOW}$ instability of (TMTSF) ${ }_{2} \mathrm{PF}_{6}$ with the $2 \mathrm{k}_{\mathrm{F}}$ BOW instability of TMTSF-DMTCNQ which diverges at the $T_{\mathrm{P}}=42 \mathrm{~K}$ Peierls transition [36]. This comparison is all the more justified because, both in (TMTSF) ${ }_{2} \mathrm{PF}_{6}$ and in TMTSF-DMTCNQ, the $2 \mathrm{k}_{\mathrm{F}}$ BOW instability develops on the same TMTSF stack which presents similar electronic characteristics in the two salts (same bandwidth, same band filling and comparable dimensionless Luttinger parameter K $\rho \sim 0.25$ [8]).

Figure 2 quantitatively compares the thermal dependence of the $1 \mathrm{D} 2 \mathrm{k}_{\mathrm{F}} \mathrm{BOW}$ fluctuations of (TMTSF) ${ }_{2} \mathrm{PF}_{6}$ [40] and of TMTSF-DMTCNQ [36,42]. Part (a) shows the $2 \mathrm{k}_{\mathrm{F}}$ susceptibility, $\chi\left(2 k_{\mathrm{F}}\right)$, given by $\mathrm{I}(\mathrm{Q}) / T$ in the expressions $(1)+(2)$, while part (b) shows the inverse intra-chain correlation length $\xi_{\mathrm{a}}{ }^{-1}$, given by expression (3). Above $\sim 100 \mathrm{~K} \chi\left(2 k_{\mathrm{F}}\right)$ behaves similarly in the two salts. Below $100 \mathrm{~K} \chi\left(2 k_{\mathrm{F}}\right)$ of TMTSF-DMTCNQ grows rapidly and diverges at $T_{\mathrm{P}}$ while $\chi\left(2 k_{\mathrm{F}}\right)$ of $(\mathrm{TMTSF})_{2} \mathrm{PF}_{6}$ saturates before decreasing below $\sim 50 \mathrm{~K}$. Above $\sim 150 \mathrm{~K} \xi_{\mathrm{a}}^{-1}$ of TMTSF-DMTCNQ and of (TMTSF) ${ }_{2} \mathrm{PF}_{6}$ are comparable. Below this temperature $\xi_{\mathrm{a}}^{-1}$ of TMTSF-DMTCNQ drops rapidly to reach zero at $\mathrm{T}_{\mathrm{P}}$ while $\xi_{\mathrm{a}}^{-1}$ of (TMTSF) ${ }_{2} \mathrm{PF}_{6}$ decreases very slowly.

The thermal dependence of $\xi_{\mathrm{a}}$ of TMTSF-DMTCNQ (named $\xi_{/ \mathrm{T}}$ below) has been quantitatively analyzed in [42] using exact calculations of the fluctuations of the Peierls chain (black continuous line of Figure $2 \mathrm{~b}$ ). Upon cooling, $\xi_{/ \mathrm{T}}$ is governed by the diverging growth of intra-chain electron-hole pair correlations associated with the increase of the thermal life time of the individual electron-hole pairs. In (TMTSF) ${ }_{2} \mathrm{PF}_{6}$ the saturation in the growth of $\xi_{\mathrm{a}}$ upon cooling means that the low temperature divergence of $\xi_{/ T}$ expected for a standard Peierls chain is capped by a cut off length. We associate at this cut off length a "mean free path" $l=\mathrm{v}^{*} \tau$, where $\tau$ corresponds to a non thermal lifetime of the electron-hole pair, and where $\mathrm{v}^{*}$ is the "charge velocity" depending of the microscopic parameters of the TMTSF electron gas ( $\mathrm{v}^{*}$ amounts to $\sim \mathrm{v}_{\mathrm{F}} / 2$ in TMTSF-DMTCNQ [42]). By adding these two independent contributions in quadrature:

$$
\xi_{\mathrm{a}}^{-2}=\xi_{/ \mathrm{T}}^{-2}+l^{-2}
$$

$l^{-1}$ can be obtained if one assumes that $\xi_{/ T}^{-1}$ is given by $\xi_{\mathrm{a}}^{-1}$ measured in TMTSF-DMTCNQ. Figure $2 \mathrm{~b}$ gives the thermal dependence of $l^{-1}$, deduced from expression (4), for (TMTSF) ${ }_{2} \mathrm{PF}_{6} . l^{-1}$ 
increases upon cooling and saturates below $100 \mathrm{~K}$, as does $\chi\left(2 k_{\mathrm{F}}\right)$. The life time $\tau$ smoothes the $\mathrm{q}$ dependence of the CDW response function near $2 \mathrm{k}_{\mathrm{F}}$, with the result to kill the low temperature divergence of both $\xi_{\mathrm{a}}$ and $\chi\left(2 k_{\mathrm{F}}\right)$.

Figure 2. Thermal dependence of (a) the $2 \mathrm{k}_{\mathrm{F}}$ BOW susceptibility, $\chi\left(2 \mathrm{k}_{\mathrm{F}}\right)$; and (b) the inverse intra-chain correlation length $\xi_{\mathrm{a}}^{-1}$ in (TMTSF) ${ }_{2} \mathrm{PF}_{6}$ and in TMTSF-DMTCNQ. In (b) the thermal dependence of the inverse mean free path $l^{-1}$ of the electron-hole pairs of (TMTSF) ${ }_{2} \mathrm{PF}_{6}$, given by (4), is given by the blue line.

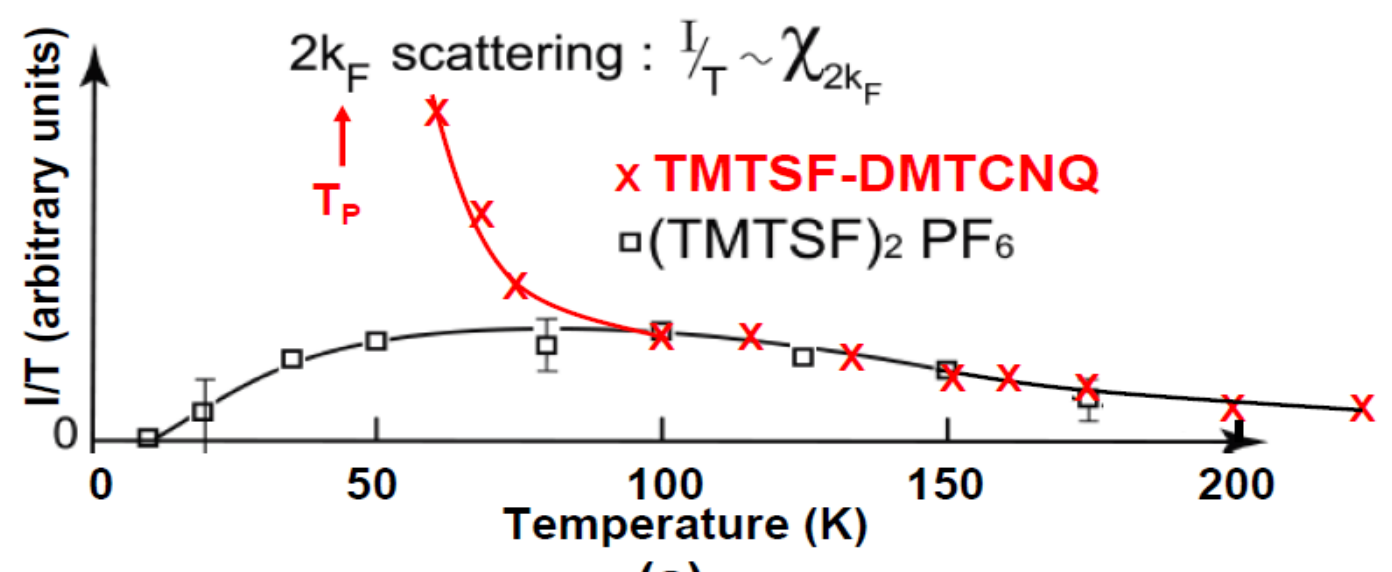

(a)

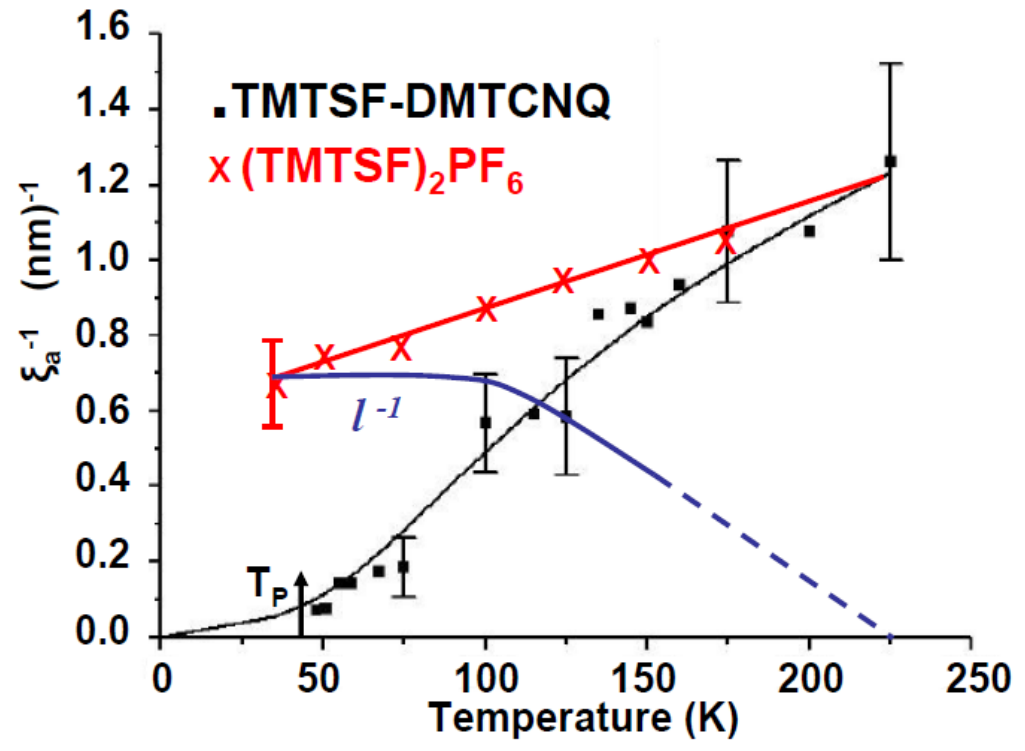

(b)

$l$ saturates at about $14 \AA$, which corresponds to one wave length $2 \mathrm{a}$ of the $2 \mathrm{k}_{\mathrm{F}} \mathrm{BOW}$. From this length one deduces an inverse electron-hole life time $\hbar / \tau=\hbar v^{*} l^{-1}$ of $43 \mathrm{meV}$. This value is one order of magnitude larger than the phonon frequency, $\hbar \Omega=5 \mathrm{meV}$, of the transverse acoustic branch which bears the Kohn anomaly in TTF-TCNQ [37]. As $\Omega \tau \ll<1$ the $2 \mathrm{k}_{\mathrm{F}}$ BOW dynamics of (TMTSF) ${ }_{2} \mathrm{PF}_{6}$ should occur in the anti-adiabatic limit and thus a Kohn anomaly should not form in the phonon spectrum. In this regime the dynamics is achieved by the critical growth of quasi-static $2 \mathrm{k}_{\mathrm{F}}$ fluctuations which are visualized in reciprocal space by the presence of $2 \mathrm{k}_{\mathrm{F}} \mathrm{X}$-ray diffuse lines above $30 \mathrm{~K}$.

The life time $\tau$ of the electron-hole pairs could be caused by the orientation disorder of the anion. The change of orientation of the anion in its cavity should break both the short F...Se contact distance 
and the weak $\mathrm{F} \ldots \mathrm{H}-\mathrm{CH}_{2}$ bonds (see Section 2.4). This will respectively modulate directly and indirectly (via the polarization of the $\mathrm{H}$ bonds and of the $\sigma$ intra-molecular electrons [21]) the Hartree potential experienced by the $\pi$ holes. These fluctuations of the Hartree potential should provide new scattering mechanisms of holes and electrons at the Fermi level, resulting in either an incoherent interstack charge transfer and/or intra-stack backward and forward scatterings. The inter-stack transfer and the backward scattering change the direction of the $\mathrm{k}_{\mathrm{F}}$ wave vector of the electron or of the hole of a pre-formed $2 \mathrm{k}_{\mathrm{F}}$ electron-hole pair. This change breaks the pairing, and thus reduces the lifetime $\tau$ of the electron-hole pairs.

Using the value at saturation of $l$, the FS should be broadened by $\hbar / \tau \sim 43 \mathrm{meV}$. This lifetime broadening amounts to the warping of the Fermi surface due to coherent interchain hopping $\left(2 \mathrm{t}_{\mathrm{b}}=44 \mathrm{meV}\right.$ from the transverse plasma edge measurement at $25 \mathrm{~K}$ [22]). Thus, the warping of the FS should be smeared out by this lifetime effect on all the temperature range of observation of the $2 \mathrm{k}_{\mathrm{F}}$ BOW diffuse lines. In this framework it has been suggested from the absence of Drude absorption in intra-chain optical reflectance of (TMTSF $)_{2} \mathrm{ClO}_{4}$ that the single particle transport is diffusive in all directions and temperatures and that the high de conductivity in chain direction is due to collective sliding CDW [43]. Similar features are observed in (TMTSF) ${ }_{2} \mathrm{PF}_{6}$ [44], but the optical spectrum has been interpreted differently [45].

Note that this lifetime effect should similarly affect the $2 \mathrm{k}_{\mathrm{F}}$ SDW fluctuations also driven by the divergence of the electron-hole response function of the correlated electron gas. In particular a reduced SDW correlation length, similarly to $\xi(\mathrm{T})$ of the $2 \mathrm{k}_{\mathrm{F}}$ BOW fluctuations reported in Figure $2 \mathrm{~b}$, should control the spatial dependence of the $2 \mathrm{k}_{\mathrm{F}}$ SDW fluctuations. Such SDW fluctuations have been probed by NMR [46] which however does not give any information on their spatial extent.

The $T_{\mathrm{N}}=12 \mathrm{~K} \mathrm{SDW}$ transition of $(\mathrm{TMTSF})_{2} \mathrm{PF}_{6}$ is achieved by a FS nesting process (see next section). However FS nesting should provide an efficient inter-chain coupling between density waves only if the FS is warped. Thus the setting of the inter-chain coupling requires a net sharpening of the FS upon cooling which can be achieved only if the extra scattering processes due to disorder vanish.

Evidence of a vanishing of the structural disorder comes from the thermal decrease of lattice entropy below $\sim 30-40 \mathrm{~K}$ ascribed to the anchorage of the anions to the methyl groups (see Section 2.4). The cooperative locking of $\mathrm{PF}_{6}$ due to the formation of $\mathrm{H}$-bonds with the methyl groups could explain the stiffening of the lattice revealed by the hardening of sound velocity below $\sim 45 \mathrm{~K}$ in (TMTSF) ${ }_{2} \mathrm{PF}_{6}$ [47]. Interestingly, a similar hardening of sound velocity is observed at the $24 \mathrm{~K} \mathrm{AO}$ transition of (TMTSF) ${ }_{2} \mathrm{ClO}_{4}$ when the orientation ordering of the $\mathrm{ClO}_{4}$ is accompanied by its linkage to the methyl groups (see section 7.3) [48]. This scenario is sustained by microwave transport measurements showing a dimension crossover in the charge transport at about the same temperature, $T_{\mathrm{X}} \sim 35 \mathrm{~K}[49,50]$, at which the disorder vanishes.

Note that if the transverse coupling $t_{b}^{\prime}$ is only considered, the warping of the FS and thus the 1D-2D spatial deconfinement of the electron gas should be effective at a larger $T_{X} \sim \mathrm{t}^{\prime} / \pi \sim 80 \mathrm{~K}$. However if the confinement due to electron-electron repulsions is considered the effective $t_{b}^{\prime}$ is reduced and it is estimated [51] that $T_{X}$ can be depressed below 50K. On the basis of available experimental arguments the generic phase diagram of [52] was built with $T_{X} \sim 80 \mathrm{~K}$. However the expected development of inter-chain correlations below $T_{X} \sim 80 \mathrm{~K}$ are neither detected in the $2 \mathrm{k}_{\mathrm{F}}$ BOW X-ray diffuse scattering 
experiments nor in the thermal dependence of the $2 \mathrm{k}_{\mathrm{F}}$ SDW fluctuations measured by NMR [46]. All these features show that the nature of the dimension crossover and of the deconfinement transition is unclear in the Bechgaard salts and that the value of the crossover temperature $T_{X}$ remains debated in the literature $[45,51,53]$. Our finding of additional effects due to anion disorder adds new elements at the discussion.

(TMTSF) $)_{2} \mathrm{PF}_{6}$ exhibits below $200-150 \mathrm{~K}$ critical 1D SDW fluctuations, probed by $\mathrm{T}_{1}^{-1} \mathrm{NMR}$ measurements [46]. These fluctuations occur in the same temperature range where the $1 \mathrm{D} 2 \mathrm{k}_{\mathrm{F}} \mathrm{BOW}$ fluctuations are also detected, which means that at high temperature (TMTSF) ${ }_{2} \mathrm{PF}_{6}$ exhibits both $2 \mathrm{k}_{\mathrm{F}}$ SDW and $2 \mathrm{k}_{\mathrm{F}}$ BOW instabilities. Below $T^{*} \sim 30 \mathrm{~K}$ inter-chain $2 \mathrm{k}_{\mathrm{F}}$ SDW critical fluctuations, due to the FS nesting process, develop [45] and the $2 \mathrm{k}_{\mathrm{F}}$ BOW fluctuations are no longer detected. However the precise reason of the vanishing of the BOW fluctuations below $T^{*}$ requires clarifications which will be the object of the next section.

\subsubsection{Interchain Coupling between the $2 \mathrm{k}_{\mathrm{F}}$ Density Waves and the Vanishing of the Anion Disorder}

It is now well established that the SDW transition of (TMTSF) $)_{2} \mathrm{PF}_{6}$ is due to a FS nesting because the interchain component $\mathrm{q}_{\mathrm{b}} \sim 0.20 \pm 0.05 \mathrm{~b}^{*}$ [54] $-0.24 \pm 0.03 \mathrm{~b}^{*}$ [55] of the SDW order, obtained by $\mathrm{NMR}$, is close to the best nesting wave vector $\sim 0.3 \mathrm{~b}^{*}$ of its FS determined from the $4 \mathrm{~K}$ structure [56]. In addition to this FS nesting process, inter-chain Coulomb interaction, $\mathrm{g}_{1} \perp$, provides also an efficient coupling mechanism between CDW [1]. The inter-chain coupling $\mathrm{g}_{1} \perp$ achieves the $3 \mathrm{D}$ ordering of $2 \mathrm{k}_{\mathrm{F}}$ CDW/BOW in charge transfer Peierls systems like TTF-TCNQ and TMTSF-DMTCNQ [36,38].

However there is the possibility for the Bechgaard salts, and as previously suggested for the Fabre salts [57], of conflicting interchain coupling mechanisms if Coulomb interactions, $\mathrm{g}_{1} \perp$, and FS nesting tend to promote different $\mathrm{b}^{*}$ transverse periodicities: $\mathrm{g}_{1} \perp$ (possibly mediated by the anion shift-see below) favoring $\mathrm{q}_{\mathrm{b}}=1 / 2 \mathrm{~b}^{*}$, as observed for the AO and SP modulations (see Sections 7 and 6), while the nesting of the FS favoring $\mathrm{q}_{\mathrm{b}} \sim 1 / 4 \mathrm{~b}^{*}$ as discussed above.

In all the superstructures achieving a transverse order between BOW, such as those formed at the $(1 / 2,1 / 2,1 / 2)$ AO transition [58,59] (see Section 7.2), the shift of the anion from the inversion center seems necessary to stabilize the $3 \mathrm{D}$ pattern of $2 \mathrm{k}_{\mathrm{F}}$ BOW in the Bechgaard and Fabre salts. In these superstructures:

- The $\left(2 \mathrm{k}_{\mathrm{F}}\right)^{-1}$ periodicity is achieved in stack direction by repeating periodically two neighboring maxima of hole concentration with two neighboring minima of hole concentration.

- $\quad$ The anion shift allows to achieve a 3D coupling between neighboring $2 \mathrm{k}_{\mathrm{F}} \mathrm{CDW}$ by locking a maxima of the $2 k_{\mathrm{F}} \pi$ hole $\mathrm{CDW}$ on the molecule towards which the anion approaches and concomitantly a minimum of $\pi$ hole density on the molecule that the anion leaves.

- $\quad$ By combining these two features, the 3D CDW pattern requires a synchronous staggered shift of near-neighbor anions along $\mathbf{a}$, along the $\mathbf{d}_{\mathbf{1}}=\mathbf{a}-\mathbf{b}+\mathbf{c}$ direction (which achieves the coupling of anions with the closest Se atom of the donor) and along the $\mathbf{d}_{\mathbf{2}}=\mathbf{a}+\mathbf{b}+\mathbf{c}$ direction (which achieves the coupling of the anions with the methyl groups and the polarization of $\sigma$ bonds of the donor). 
Figure 3 resumes this synchronous CDW - anion shift pattern.

Figure 3. Schematic representation in the (a, $\left.\mathbf{d}_{\mathbf{i}}\right)$ plane of the array of $2 \mathrm{k}_{\mathrm{F}} \mathrm{CDW}$ (in black) coupled to anion shifts (in green). Donors with an excess (defect) of hole are shown in red (blue).

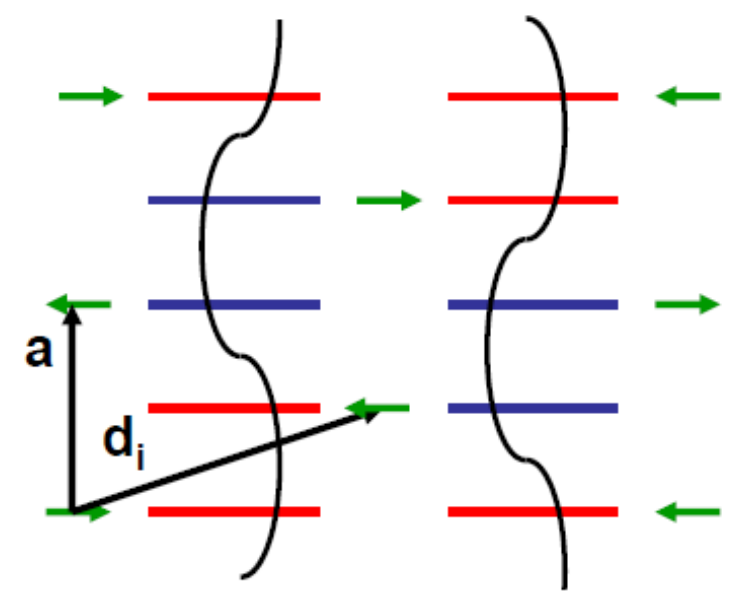

In this model the CDW wave vector $\mathbf{q}$ allowing such $\pi$ phase shifts between the anion displacements is given by:

$$
\begin{gathered}
\mathbf{q a}=\pi \text { and } \mathbf{q} \mathbf{d}_{\mathbf{i}}=\mathbf{q}(\mathbf{a} \pm \mathbf{b}+\mathbf{c})=\pi(\bmod .2 \pi), \text { which leads to: } \\
\mathrm{q}_{\mathrm{a}}=1 / 2 \text { and } \mathrm{q}_{\mathrm{a}} \pm \mathrm{q}_{\mathrm{b}}+\mathrm{q}_{\mathrm{c}}=1 / 2(\bmod .1)
\end{gathered}
$$

The relationships (5) lead to $\pm \mathrm{q}_{\mathrm{b}}+\mathrm{q}_{\mathrm{c}}=0$ (mod.1) which has two solutions: $\mathrm{q}_{\mathrm{b}}=\mathrm{qc}=1 / 2$ and $\mathrm{q}_{\mathrm{b}}=\mathrm{qc}=0$. The first solution $(1 / 2,1 / 2,1 / 2)$ corresponds to the $2 \mathrm{k}_{\mathrm{F}}$ BOW/AO modulation, $\mathbf{q}_{\mathrm{AO}}$, (see Section 7.2) and the SP modulation, $\mathbf{q}_{\mathrm{SP}}$ (see Section 6). The second solution $(1 / 2,0,0)$ corresponds to the $2 \mathrm{k}_{\mathrm{F}}$ BOW/AO modulation in the $\mathrm{NO}_{3}$ salts (see Section 7.5). As usual the $2 \mathrm{k}_{\mathrm{F}} \mathrm{BOW}$ has a phase shift of $\pi / 2$ with the $2 \mathrm{k}_{\mathrm{F}}$ CDW. In [60] this model is used to derive the anion shift pattern setting the $4 \mathrm{k}_{\mathrm{F}} \mathrm{CDW}$ or $\mathrm{CO}$.

In this picture the suppression of the $2 \mathrm{k}_{\mathrm{F}}$ BOW at low temperature could be explained by the impossibility to establish an inter-chain coupling between CDW via the anion shift. The absence of a low temperature anion shift, ascribed the lock-in of the $\mathrm{PF}_{6}$ to the methyl groups via the formation of H-bonds, was previously discussed in Sections 2.4 and 3.1.1.

\subsection{Evolution of the $2 \mathrm{k}_{F} B O W$ Instability along the TMTSF-TMTTF Series}

The $1 \mathrm{D} 2 \mathrm{k}_{\mathrm{F}}$ BOW instability of (TMTSF) $)_{2} \mathrm{ClO}_{4}$ [40] behaves as the one of (TMTSF) ${ }_{2} \mathrm{PF}_{6}$ and $\mathrm{AsF}_{6}$. Figure 4 reports the thermal dependence of the $2 \mathrm{k}_{\mathrm{F}}$ peak intensity, $\mathrm{I}\left(2 \mathrm{k}_{\mathrm{F}}\right)$ given by expression (1), of the $\mathrm{X}$-ray diffuse scattering from (TMTSF $)_{2} \mathrm{ClO}_{4}$. In this salt the low temperature vanishing of $\mathrm{I}\left(2 \mathrm{k}_{\mathrm{F}}\right)$ has to be associated with the $3 \mathrm{D}$ critical growth below $\sim 40 \mathrm{~K}$ of the $(0,1 / 2,0)$ AO structural instability promoting the uniform $\mathrm{ClO}_{4}$ ordering and anion shift in stack direction (see Section 7.3) [61]. Figure 4 shows surprisingly that the thermal dependence of the $2 \mathrm{k}_{\mathrm{F}}$ BOW fluctuations of (TMTSF) ${ }_{2} \mathrm{ClO}_{4}$ is strongly modified upon substitution by the TMTTF [62]. 
Figure 4. Thermal dependence of the $2 \mathrm{k}_{\mathrm{F}}$ peak intensity, $\mathrm{I}\left(2 k_{\mathrm{F}}\right)$, in the solid solution $\left[(\mathrm{TMTSF})_{1-x}(\mathrm{TMTTF})_{x}\right]_{2} \mathrm{ClO}_{4}$ for $x=0$ (black squares), $0.5 \%$ (red circles) and $30 \%$ (green triangles) of TMTTF deduced from the data reported in [40] and [62].

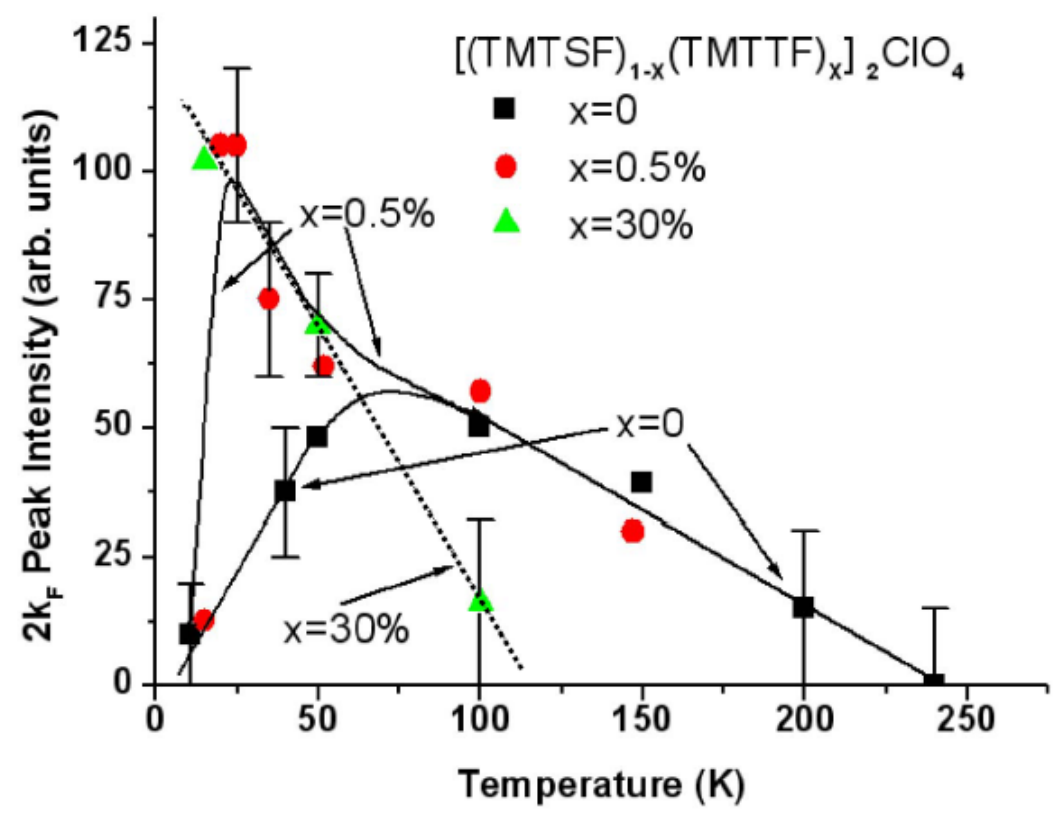

A low temperature divergence of $\mathrm{I}\left(2 k_{\mathrm{F}}\right)$ appears for very small amount of TMTTF substituent. For $x=0.5 \%$ this additional divergence pushes down at lower temperatures the drop of $\mathrm{I}\left(2 \mathrm{k}_{\mathrm{F}}\right)$ which remains due to the competing AO instability as in pure (TMTSF $)_{2} \mathrm{ClO}_{4}$. The thermal behavior of $\mathrm{I}\left(2 \mathrm{k}_{\mathrm{F}}\right)$ in the $x=0.5 \%$ salt resembles the one observed (see Figure 2 in [63]) in the (TMDTDSF) ${ }_{2} \mathrm{PF}_{6}$ isostructural salt built on a donor hybrid between the TMTSF and TMTTF molecules. However in (TMDTDSF $)_{2} \mathrm{PF}_{6}$ the drop of $\mathrm{I}\left(2 \mathrm{k}_{\mathrm{F}}\right)$ below $20 \mathrm{~K}$ is not due to an $\mathrm{AO}$ transition but, as for (TMTSF) ${ }_{2} \mathrm{PF}_{6}$, to the stabilization of a SDW ground state below $7 \mathrm{~K}$. Note that because of the orientation disorder of the TMDTDSF molecule in the structure [64], $\mathrm{I}\left(2 \mathrm{k}_{\mathrm{F}}\right)$ does not vanish completely at low temperature in (TMDTDSF) ${ }_{2} \mathrm{PF}_{6}$ so that a $1 \mathrm{D}$ SP short range order (on $\xi_{\mathrm{a}} \sim 25 \AA$ ) still coexists with the $2 \mathrm{k}_{\mathrm{F}}$ SDW modulation.

Only the low temperature divergence of $\mathrm{I}\left(2 \mathrm{k}_{\mathrm{F}}\right)$ remains in the $x=30 \%$ salt below $100 \mathrm{~K}$. This low temperature divergence of the $2 \mathrm{k}_{\mathrm{F}}$ diffuse scattering intensity recalls the divergence of the SP fluctuations observed in (TMTTF $)_{2} \mathrm{Br}$ and $\mathrm{PF}_{6}$ below $80 \mathrm{~K}$ and $60 \mathrm{~K}$ respectively [65].

In TMTTF salts the occurrence of a low temperature divergence of $\mathrm{I}\left(2 \mathrm{k}_{\mathrm{F}}\right)$ coincides with the presence of a high temperature charge localization [51,52] which leaves the spin degrees of freedom available for a low temperature SP or AF instability. This charge localization manifests below a temperature, $T_{\rho}$ [66], higher than the onset temperature of the critical growth of the SP fluctuations. In the $x=30 \%$ salt the $2 \mathrm{k}_{\mathrm{F}}$ fluctuations detected below the spin-charge separation temperature $T_{\mathrm{\rho}}$ [67] are thus the structural fingerprint of an incipient SP instability which fully diverges in (TMTTF $)_{2} \mathrm{PF}_{6}$.

In conclusion the $\left[(\mathrm{TMTSF})_{1-x}(\mathrm{TMTTF})_{x}\right]_{2} \mathrm{ClO}_{4}$ solid solution illustrates quite well when $x$ increases that the evolution from a $2 \mathrm{k}_{\mathrm{F}}$ BOW instability, which vanishes at low temperature, to a divergent $\mathrm{SP}$ instability follows the growth of the $4 \mathrm{k}_{\mathrm{F}}$ charge localization 


\subsection{The Spin-Peierls Instability}

(TMTTF) ${ }_{2} \mathrm{PF}_{6}$ develops $2 \mathrm{k}_{\mathrm{F}} \mathrm{SP}$ critical fluctuations below about $60 \mathrm{~K}$ (taken as the mean field transition temperature of the SP transition, $T_{\mathrm{SP}}{ }^{\mathrm{MF}}$ ). The critical nature of the SP fluctuations is assessed by the divergent growth of the SP susceptibility, $\chi\left(2 \mathrm{k}_{\mathrm{F}}\right)[7,8,65]$, and of the intra-chain correlation length, $\xi_{\mathrm{SP}}$; both quantities being defined by expression (3). The divergence is achieved at $T_{\mathrm{SP}} \sim 17 \mathrm{~K}$, temperature at which a long range $(1 / 2,1 / 2,1 / 2) \mathrm{SP}$ stack tetramerization occurs [68].

Figure 5 compares the thermal dependence of $\xi_{\mathrm{SP}}{ }^{-1}$ of (TMTTF $)_{2} \mathrm{PF}_{6}$ and of (TMTTF) $)_{2} \mathrm{Br}$. It clearly shows that the SP fluctuations of (TMTTF) ${ }_{2} \mathrm{Br}$ do not diverge. Thus Figure 5 resembles for the SP fluctuations of (TMTTF $)_{2} \mathrm{Br}$ at Figure $2 \mathrm{~b}$ for the $2 \mathrm{k}_{\mathrm{F}} \mathrm{BOW}$ fluctuations of (TMTSF) ${ }_{2} \mathrm{PF}_{6}$. We shall consider at the end of this section this aspect of the SP fluctuations of (TMTTF) ${ }_{2} \mathrm{Br}$.

Figure 5. Thermal dependence of the inverse intra-chain correlation length $\xi_{\mathrm{SP}}{ }^{-1}$ of the SP instability of (TMTTF) ${ }_{2} \mathrm{PF}_{6}$ (red dots) and of (TMTTF) ${ }_{2} \mathrm{Br}$ (blue crosses). $T_{\mathrm{SP}}{ }^{\mathrm{MF}}$ and $\xi_{0}$ are indicated in the lower panel for each salt. The continuous line for the $\mathrm{PF}_{6}$ salt is $\xi_{\mathrm{SP}}{ }^{-1}$ calculated [69] for the weakly localized SP chain.

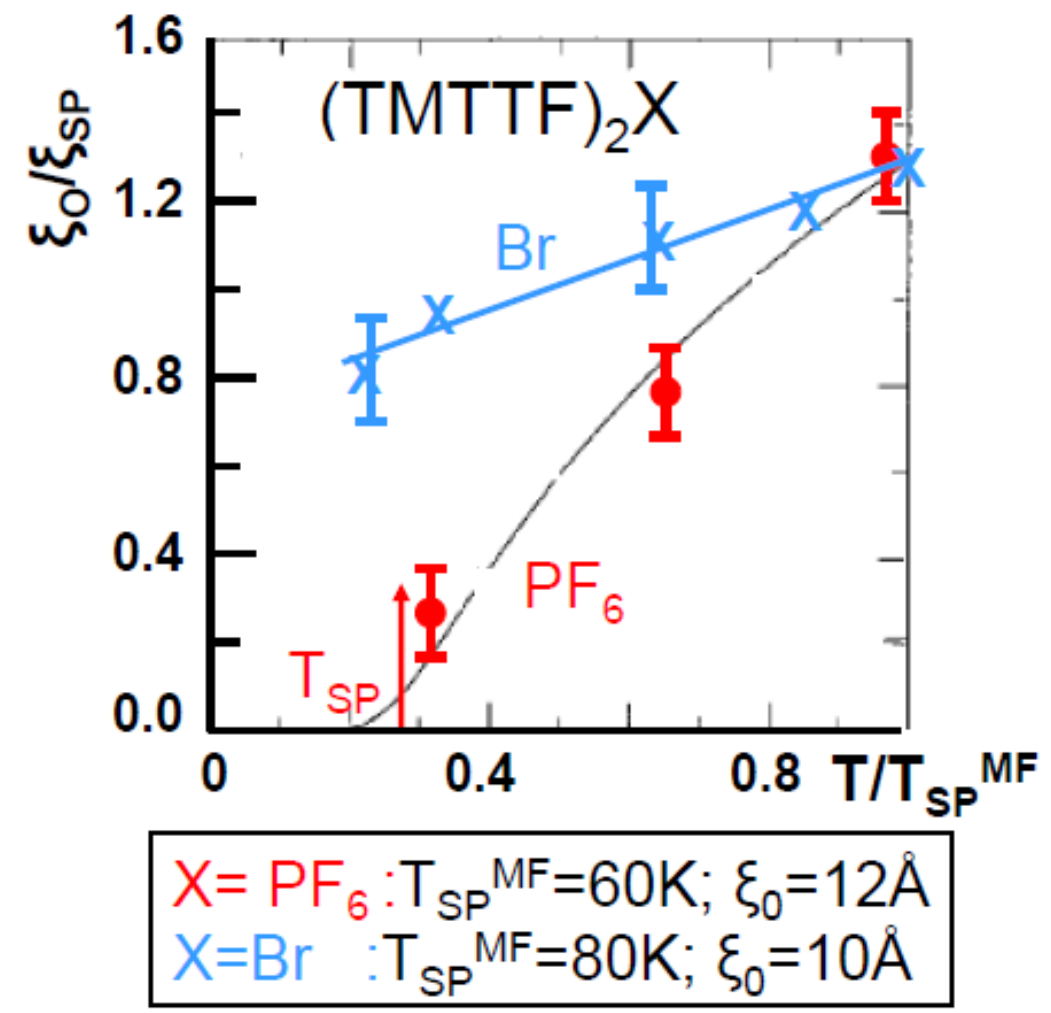

The thermal dependence of $\xi_{\mathrm{SP}}{ }^{-1}$ of (TMTTF) ${ }_{2} \mathrm{PF}_{6}$ can be well accounted for by the calculation of the fluctuations of the weakly localized SP chain [69]. From this calculation one deduces that the SP coherence length $\xi_{0}$ amounts to $12 \AA$. This value is very close to $\xi_{0} \sim 10 \AA$ found for the SP instability of (BCPTTF $)_{2} \mathrm{PF}_{6}$ [7]. From the relationship linking $\xi_{0}$ to $T_{\mathrm{SP}}{ }^{\mathrm{MF}}$ :

$$
\xi_{0}=\hbar v_{\sigma} / \pi k_{\mathrm{B}} T_{\mathrm{SP}} \mathrm{MF}
$$


one gets a "spin velocity" $\hbar v_{\sigma} \sim 0.2 \mathrm{eV} \AA$ in $(\mathrm{TMTTF})_{2} \mathrm{PF}_{6}$. A similar value $\hbar v_{\sigma}=0.27 \mathrm{eVA}$ is obtained in $(\mathrm{BCPTTF})_{2} \mathrm{PF}_{6}$ where $T_{\mathrm{SP}}^{\mathrm{MF}} \sim 100 \mathrm{~K}$. For this last compound which can be described by the Heisenberg Hamiltonian coupling well localized spins one gets, with $\hbar \mathrm{v}_{\sigma}=\pi \mathrm{Ja} / 2$, an AF exchange coupling $\mathrm{J}=280 \mathrm{~K}$ which is close to $\mathrm{J}=330 \mathrm{~K}$ deduced from the fit of the thermal dependence of the spin susceptibility of $(\mathrm{BCPTTF})_{2} \mathrm{PF}_{6}[70]$. If the same analysis is performed for $(\mathrm{TMTTF})_{2} \mathrm{PF}_{6}$, where the holes are less localized than in $(\mathrm{BCPTTF})_{2} \mathrm{PF}_{6}$, one gets, using the above quoted $\hbar v_{\sigma}$ value, $\mathrm{J}=210 \mathrm{~K}$, which is twice smaller than $\mathrm{J}=420 \mathrm{~K}$ obtained from the fit of the thermal dependence of the spin susceptibility [71]. A similar discrepancy is obtained for (TMTTF) ${ }_{2} \mathrm{Br}$ where expression (6) leads, with $\xi_{0} \sim 10 \AA$ and $T_{\mathrm{SP}}^{\mathrm{MF}} \sim 80 \mathrm{~K}$, to a $\mathrm{J}$ value of $230 \mathrm{~K}$ twice smaller than $\mathrm{J}=500 \mathrm{~K}$ obtained from the fit of the thermal dependence of the spin susceptibility [71]. It thus appears that the analysis using the Heisenberg Hamiltonian, strictly valid for localized spins, does not hold for the TMTTF salts where the holes bearing the spins are too delocalized.

Let us remark that in the TMTTF salts $k_{B} T_{\mathrm{SP}}{ }^{\mathrm{MF}}$ amounts to the energy of the transverse acoustic phonon mode, $\hbar \Omega \sim 5 \mathrm{meV}$, which controls the dynamics of the SP instability. With $k_{B} T_{\mathrm{SP}}{ }^{\mathrm{MF}} \sim \hbar \Omega$, the SP transition should occur in the adiabatic limit with however a strongly damped soft mode (see Figure $4 \mathrm{~b}$ in [72]). This is consistent with the adiabatic limit used in [69] for the calculation of $\xi_{\mathrm{SP}}{ }^{-1}$ shown in Figure 5. However with $k_{B} T_{\mathrm{SP}}^{\mathrm{MF}} \sim \hbar \Omega$, (TMTTF) ${ }_{2} \mathrm{PF}_{6}$ should not be too far from the adiabaticantiadiabatic crossover. As $T_{\mathrm{SP}}{ }^{\mathrm{MF}}$ decreases when the size of the anion increases, the $\mathrm{AsF}_{6}$ salts could be located in the anti-adiabatic regime and the $\mathrm{SbF}_{6}$ salt in the gapless regime where the SP ground state cannot be stabilized (see Section 6.2 and the analysis performed in [73]). The location of the $\mathrm{SbF}_{6}$ salt in the gapless regime agrees with the non detection of SP X-ray diffuse scattering fluctuations in this salt. Due to the absence of a SP instability the $\mathrm{SbF}_{6}$ salt undergoes an AF transition. The SCN salt behaves similarly. However the mutual exclusion between AF and SP instabilities is not general among the TMTTF salts because the Br salt exhibits below $80 \mathrm{~K}$ quasi-1D SP fluctuations which coexist with AF fluctuations whose divergence [74] stabilizes below $\mathrm{T}_{\mathrm{N}}=13 \mathrm{~K}$ the AF ground state.

As previously mentioned, the thermal dependence of the SP fluctuations of (TMTTF) ${ }_{2} \mathrm{Br}$ resembles those of the $2 \mathrm{k}_{\mathrm{F}} \mathrm{BOW}$ fluctuations of (TMTSF) $)_{2} \mathrm{PF}_{6}$. Figure 5 shows that $\xi_{\mathrm{SP}}$ reaches $\sim 13 \AA$ before the vanishing of the SP fluctuations, a similar value of $\xi_{\mathrm{BOw}}\left(14 \AA\right.$ ) was found in (TMTSF) ${ }_{2} \mathrm{PF}_{6}$ (Figure $2 b$ ). The vanishing of the SP fluctuations in the Br salt could have the same origin as for the BOW fluctuations of (TMTSF) ${ }_{2} \mathrm{PF}_{6}$. However there is no low temperature structural refinement in the $\mathrm{Br}$ salt allowing sustaining this assertion. Such a structure would be all the more desirable than thermal expansion measurements [23] show that the lattice (as well as electronic properties, see Section 4.1) exhibits unusual features just above $T_{\mathrm{N}}$. Upon cooling below $18 \mathrm{~K} \alpha_{\mathrm{c}^{*}}$ abruptly drops, exhibits a negative maximum around $17 \mathrm{~K}$, then gently increases towards zero. These low temperature thermal expansion anomalies, which recall glassy phenomena involving ethylene groups in (BEDT-TTF) 2 X [3], could be also associated to the freezing of the disorder of methyl groups of the TMTTF.

\section{The Magnetic Ground States of the Bechgaard and Fabre Salts}

At ambient pressure $(\mathrm{TMTTF})_{2} \mathrm{Br}$ undergoes a commensurate $\mathrm{AF}$ order at $T_{\mathrm{N}}=13 \mathrm{~K}$ while $(\mathrm{TMTSF})_{2} \mathrm{PF}_{6}$ undergoes an incommensurate SDW order at $\mathrm{T}_{\mathrm{SDW}}=12 \mathrm{~K}$. Pressure studies show that the AF ground state evolves into a SDW ground state when the electron localization (i.e., $T_{\rho}$ ), 
occurring on the TMTTF side of the generic phase diagram drops [51,52]. This section will show that, because of the presence of a sizeable magneto-elastic coupling, the magnetic ground states of the Fabre and Bechgaard salts, which involve also structural degrees of freedom [65], are quite a bit more complex than those expected for a simple magnetic transition. This magneto-elastic coupling is also responsible of the occurrence of SP transitions which will be considered in Section 6.

\subsection{The AF Phase of $(\text { TMTTF })_{2} B r$ and the Pre-Transitional Anomalies}

AF order which develops below a 2 nd order phase transition in $(\mathrm{TMTTF})_{2} \mathrm{Br}$ stabilizes a $\mathrm{q}_{\mathrm{AF}}=(1 / 2,1 / 4$, ?) commensurate magnetic modulation [75]. The magnetic order is accompanied by a structural modulation at the $\mathrm{q}_{\mathrm{S}}=(1,1 / 2, ?)$ reduced wave vector (the component $1 \mathrm{a}^{*}$, corresponding to the $4 \mathrm{k}_{\mathrm{F}}$ wave vector, means that the superstructure reflections are detected in $\mathrm{H}$ odd layers of Bragg reflections [65]). As $\mathrm{q}_{\mathrm{S}}=2 \mathrm{q}_{\mathrm{AF}}$ the structural modulation should result from a "magneto-elastic" coupling which will be considered below.

The coupling between the structural and AF order parameters is analyzed in Annex A.1 using the simplest Landau development of the free energy. Its minimization gives two solutions shown in Figure 6.

Figure 6. (a) Mixed AF-CO order where the hole rich molecule bears the spin and the hole poor molecule is a node of the magnetization wave; (b) AF modulation where each site bears magnetization and the same charge. In (b) the nodes of the magnetization wave are located on one bond out of two. The spin direction is given by the sign of $\eta(q)$ defined in Annex A.1. In (a), the charge rich/poor $(+\rho /-\rho)$ sites are represented by the red/blue circles of a different size. The dimerization of the stack is ignored in this figure.

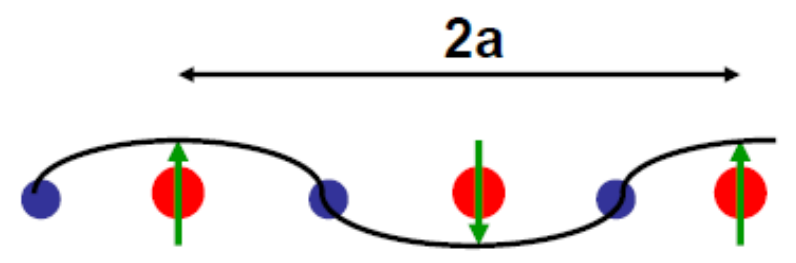

(a)

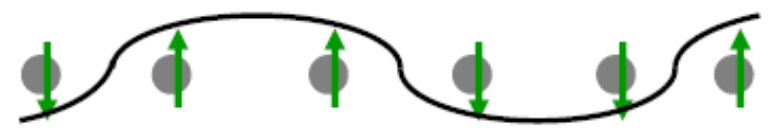

(b)

Solution (a) corresponds to an AF order with one site out of two bearing the magnetization, while solution (b) gives an AF order where all the sites bear the magnetization. In the Landau theory of Annex A.1 only solution (a) gains energy by setting a $4 \mathrm{k}_{\mathrm{F}}$ structural modulation. NMR lineshape analysis [75] better agrees with the spin configuration shown in (a). In the schematic representation of Figure 5a the presence of a spin on one site out of two is accompanied by an excess of hole on the magnetic site and a defect of hole on the non magnetic site. This leads to the formation of a $4 \mathrm{k}_{\mathrm{F}} \mathrm{CDW}$ or CO [76]. CO should be accompanied by a $4 \mathrm{k}_{\mathrm{F}}$ internal deformation wave of the TMTTF molecules in phase with the charge density per molecule. The CO pattern could be also stabilized by a 
displacement wave of the $\mathrm{Br}$ towards the molecule bearing the excess of hole (if there is no H-bonds blocking the anion shift), recalling the one found in the structural refinement of $\delta$-(EDT-TTF$\left.\mathrm{CONMe}_{2}\right)_{2} \mathrm{Br}$ in its $\mathrm{CO}$ ground state [77]. As previously mentioned in Section 3.3, (TMTTF) ${ }_{2} \mathrm{Br}$ exhibits unusual features few degrees above $T_{\mathrm{N}}$. In addition to the lattice thermal expansion anomaly previously reported, (TMTTF) ${ }_{2} \mathrm{Br}$ exhibits below $T^{*} \sim 18-22 \mathrm{~K}$ anomalous electronic properties:

- The thermal dependence of the electrical conductivity exhibits, depending on the measurements, either a kink at $T^{*}$ or a slope anomaly at $T^{*}$, or even a plateau below $T^{*}$ (to my knowledge the $T^{*}$ anomaly was first reported in Figure 1 of [78]).

- The spin susceptibility abruptly decreases below $T^{*}[79,80]$; this effect is apparently not due to the formation of a pseudo gap because SP fluctuations vanish also below $T^{*}$.

- A broadening of the NMR spectra, interpreted as being due to non-homogeneities in the electronic states, is observed below $T^{*}[80]$.

$(\mathrm{TMTTF})_{2} \mathrm{Br}$ bears some resemblance with (TMTTF) $)_{2} \mathrm{SCN}$ which also stabilizes the same modulations. (TMTTF) ${ }_{2} \mathrm{SCN}$ (see Section 7.4) sets at $T_{\mathrm{CO}}=160 \mathrm{~K}$ an anti-ferroelectric $(1,1 / 2,1 / 2) \mathrm{CO}$ superstructure followed at $T_{\mathrm{N}}=7 \mathrm{~K}$ by a $\mathrm{q}_{\mathrm{AF}}=(1 / 2,1 / 4, ?)$ magnetic order while in (TMTTF $)_{2} \mathrm{Br}$ both modulations are established at the same $13 \mathrm{~K}$ transition. This analogy is better revealed by dielectric permittivity measurements showing in both salts upon cooling an increase of dielectric constant, probably due to the growth of $\mathrm{CO}$ ferroelectric segments on individual stacks, followed by an abrupt drop of the dielectric constant $\sim 20 \mathrm{~K}$ above $T_{\mathrm{CO}}$ in (TMTTF) ${ }_{2} \mathrm{SCN}$ [81] and below $\sim T^{*}$ in (TMTTF) ${ }_{2} \mathrm{Br}$ [82], probably when the inter-chain anti-ferroelectric coupling develops. 3D CO local order should induce the charge non homogeneities revealed by NMR [80] below $T^{*}$ in (TMTTF) ${ }_{2} \mathrm{Br}$. These charge non homogeneities could trigger local random $\mathrm{Br}$ displacements, possibly inducing methyl group disorder, which could cause the lattice expansion anomaly observed along the $c^{*}$ direction [23].

\subsection{The SDW Phase of $(T M T S F)_{2} P F_{6}$}

$(\mathrm{TMTSF})_{2} \mathrm{PF}_{6}$ stabilizes below $T_{\mathrm{SDW}}=12 \mathrm{~K}$ an incommensurate $\mathrm{q}_{\mathrm{SDW}}=(1 / 2, \sim 1 / 4$, ?) magnetic modulation [54,55] stabilized by the nesting of its FS [56] and where the exchange field due to the intra-site coulomb repulsion $U$ opens a full gap at the Fermi energy. However the phase transition of (TMTSF) ${ }_{2} \mathrm{PF}_{6}$ exhibits additional features. First, the $2 \mathrm{k}_{\mathrm{F}} \mathrm{SDW}$ order is stabilized by a first order transition [83], while the Peierls or Slater transition caused by the simple divergence of CDW/BOW or SDW electron-hole response functions is of second order [1,2]. It follows that the order parameter, the magnetization [71] or the square root of the $2 \mathrm{k}_{\mathrm{F}}$ satellite intensity [65] (see below), does not continuously vanish to zero at $\mathrm{T}_{\mathrm{SDW}}$. There is also a jump of electrical conductivity at the MI transition. Second, a "specific heat" anomaly is observed at $\mathrm{T}_{\mathrm{SDW}}$ in thermal expansion measurements [25]; a feature not expected for a pure electronic SDW transition. Third, very weak X-ray satellite reflections (but too intense to be due to magnetic scattering) are observed below $\mathrm{T}_{\mathrm{SDW}}$ at the reduced $\mathrm{q}_{\mathrm{SDW}}\left(2 \mathrm{k}_{\mathrm{F}}\right.$ CDW reflections) and $2 \mathrm{q}_{\mathrm{SDW}}\left(4 \mathrm{k}_{\mathrm{F}} \mathrm{CDW}\right.$ reflections) wave vectors [7,65,84].

Thus the ground state of (TMTSF) ${ }_{2} \mathrm{PF}_{6}$ must be described with 3 order parameters related to the $2 \mathrm{k}_{\mathrm{F}}$ SDW, $2 \mathrm{k}_{\mathrm{F}} \mathrm{CDW}$ and $4 \mathrm{k}_{\mathrm{F}} \mathrm{CDW}$ modulations. A simplified analysis of the phase transition is given Annex A.2 in the frame work of the Landau development of the free energy. This simple analysis 
shows, in agreement with experimental observations, that for an attractive enough coupling between the $2 \mathrm{k}_{\mathrm{F}}$ SDW and $2 \mathrm{k}_{\mathrm{F}}$ CDW order parameters a mixed SDW-CDW modulated ground state can be directly obtained through a first order transition.

The stabilization of a first order phase transition particularly requires that the Landau coefficients:

- $\mathrm{a}_{1}$, associated to the $2 \mathrm{k}_{\mathrm{F}} \mathrm{SDW}$ order parameter, changes of sign near $\mathrm{T}_{\mathrm{SDW}}$, which means that the $2 \mathrm{k}_{\mathrm{F}}$ SDW susceptibility, $\mathrm{a}_{1}{ }^{-1}$, should diverge. Such a divergence is detected by NMR [46].

- $\mathrm{a}_{2}$, associated to the $2 \mathrm{k}_{\mathrm{F}} \mathrm{CDW}$ order parameter, should be very small, which means that the system should present incipient $2 k_{\mathrm{F}}$ CDW instability. This is the case above $\sim 30 \mathrm{~K}$ for the $2 \mathrm{k}_{\mathrm{F}}$ BOW previously discussed in Section 3.1.

These conditions together with additional requirements given in Annex A.2, lead to phase diagrams shown in Figure 7.

Figure 7. $2 \mathrm{k}_{\mathrm{F}} \mathrm{SDW} / \mathrm{CDW}$ phase diagrams in function of the Landau coefficients $\mathrm{a}_{1}$ and $\mathrm{a}_{2}$ for an attractive interaction, $c$, between the $2 \mathrm{k}_{\mathrm{F}} \mathrm{SDW}$ and $2 \mathrm{k}_{\mathrm{F}} \mathrm{CDW}$ order parameters (adapted from Figure 3 in [85]). (a) Corresponds to $c<-2 \sqrt{ }\left(b_{1} b_{2}\right)$ and $b_{1}, b_{2}>0$ (case also displayed in Figure A.1); (b) Corresponds to $c, b_{1}<0$ and $b_{2}>0$; (c) Corresponds to $0>c$ $>-2 \sqrt{ }\left(b_{1} b_{2}\right)$ and $b_{1}, b_{2}<0$. The notations are defined in Annex A.2. Thin (thick) lines represent $2^{\text {nd }}\left(1^{\text {st }}\right)$ order transition lines. White dots indicate merging points between transition lines and black dots represent tricritical points. In each phase diagram the grey interrupted lines show a trajectory starting from the uniform phase " 0 ", crossing, after a $1^{\text {st }}$ order transition, the SDW + CDW phase to finally reach, after a 2nd order transition, a pure SDW phase.

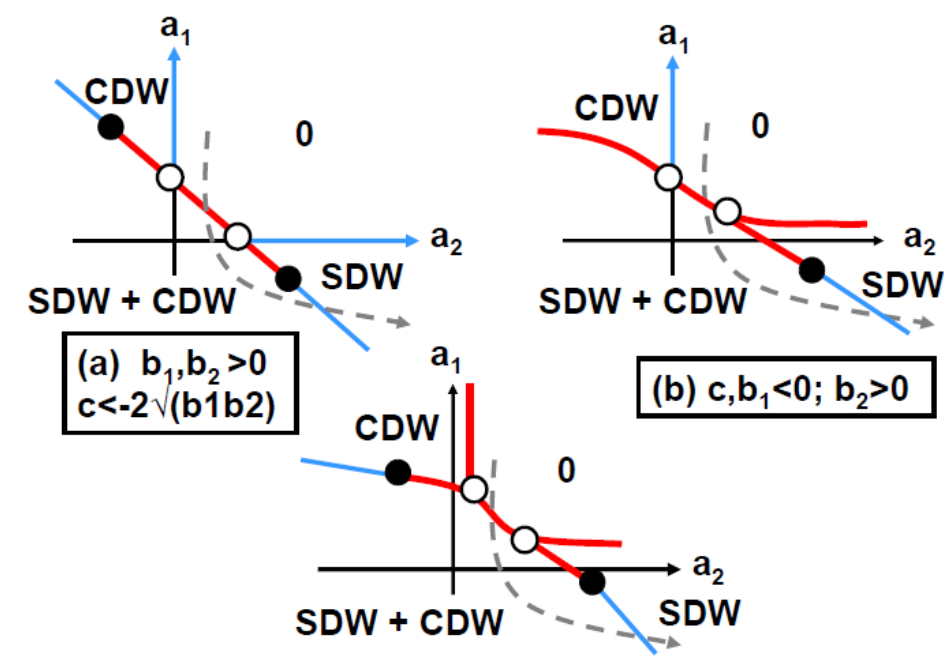

(c) $b_{1}, b_{2}<0 ; 0>c>-2 \sqrt{ }(b 1 b 2)$

The phase diagrams shown in Figure 7 leave the possibility (interrupted lines) of an additional transition at lower temperature towards a pure $2 \mathrm{k}_{\mathrm{F}}$ SDW phase. This transition could occur in (TMTSF) ${ }_{2} \mathrm{PF}_{6}$ at $\sim 3.5 \mathrm{~K}$, temperature at which earlier NMR measurements $[86,87]$ exhibit anomalies. Consistently with the occurrence of a pure SDW sub-phase, a vanishing of the $2 \mathrm{k}_{\mathrm{F}} \mathrm{CDW}$ satellite reflection intensity is observed below $\sim 3.5 \mathrm{~K}$ [84]. 
The finding of a mixed $2 k_{\mathrm{F}}$ SDW/CDW ground state implies that both $2 \mathrm{k}_{\mathrm{F}} \mathrm{SDW}$ and $2 \mathrm{k}_{\mathrm{F}}$ CDW response functions should be strong. Let us first consider the electronic phase diagram. Recent calculation $[88,89]$ shows that, in addition to the FS nesting process tending to promote, in presence of sizeable intra-chain coulomb repulsions, the $2 \mathrm{k}_{\mathrm{F}} \mathrm{SDW}$ ground state, the inter-chain backward repulsive coulomb interaction $\mathrm{g}_{1} \perp$ previously considered in Section 3.1.2 can in parallel stabilize a $2 \mathrm{k}_{\mathrm{F}} \mathrm{CDW}$ ground state for strong enough $\mathrm{g}_{1} \perp$. These calculations show that with both kinds of inter-chain coupling there is a proximity between the $2 \mathrm{k}_{\mathrm{F}}$ SDW and $2 \mathrm{k}_{\mathrm{F}}$ CDW phases, and that, even if the SDW ground state is stabilized, sizeable $2 \mathrm{k}_{\mathrm{F}} \mathrm{CDW}$ electronic correlations remain above the transition temperature. Interestingly when the FS nesting breaking effects destabilize the insulating density wave ground state, strong $\mathrm{g}_{1} \perp$ tends to stabilize triplet $\mathrm{f}$ superconductivity instead of the singlet $\mathrm{d}$ superconductivity occurring for small $\mathrm{g}_{1} \perp$ (for a recent review of the interplay between superconductivity and $2 \mathrm{k}_{\mathrm{F}}$ density waves see [90]).

However in the present case, already discussed in Section 3.1.2, $\mathrm{g}_{1} \perp$ stabilizes a different periodicity as does the FS nesting, a feature not considered in the theory performed in $[88,89]$. This competition together with the lock-in of the anion destabilizes the $2 \mathrm{k}_{\mathrm{F}}$ BOW as seen in Section 3.1. However the CDW counterpart of $2 \mathrm{k}_{\mathrm{F}}$ instability could remain active and contribute to the ground state modulation if the $3 \mathrm{D}$ ordering of CDW is achieved by the same FS nesting mechanism as for the $2 \mathrm{k}_{\mathrm{F}} \mathrm{SDW}$. As $\mathrm{g}_{1} \perp$ is not activated below $30 \mathrm{~K}$, the structural modulation observed below $\mathrm{T}_{\mathrm{SDW}}$ must not be of the BOW type (The absence of BOW structural features explains why the $2 \mathrm{k}_{\mathrm{F}}$ satellite reflections are of very weak intensity). For this reason it was proposed in [7] and [65] that the weak satellite reflections detected below $T_{\mathrm{SDW}}$ could correspond to the X-ray scattering by the electronic $2 \mathrm{k}_{\mathrm{F}} \mathrm{CDW}$. Eventually a molecular distortion could follow the setting of the $2 \mathrm{k}_{\mathrm{F}} \mathrm{CDW}$ (see below).

The separation between pure $2 \mathrm{k}_{\mathrm{F}}$ SDW and $2 \mathrm{k}_{\mathrm{F}} \mathrm{CDW}$ ground states implies that there is a natural repulsion between the associated density waves, which means that the bare coupling term $v$ introduced in the Landau free energy of Annex A.2 is repulsive. However if these two density waves are simultaneously stabilized in the same phase, the free energy is minimized if the $2 \mathrm{k}_{\mathrm{F}} \mathrm{SDW}$ and $2 \mathrm{k}_{\mathrm{F}}$ CDW are in phase quadrature ( $\omega=\pi / 2$ phase shift). This corresponds also to a $\pi / 2$ phase shift between the spin $\uparrow$ and spin $\downarrow$ components of the SDW and of the electronic CDW [8,65].

The "specific heat" anomaly observed at $T_{\mathrm{SDW}}$ in thermal expansion measurements [25] shows that the electronic CDW should have a structural counterpart. This counterpart is not a BOW modulation (see Section 3.1). However if the CDW consists primarily in a modulation of the hole occupancy on the molecules, one expects that molecular deformations should follow the electronic density wave by elongating the TMTSF hole rich molecules and contracting the TMTSF electron rich molecules. This should lead to a modification of intra-molecular vibration modes in Raman and/or infrared spectra of (TMTSF) ${ }_{2} \mathrm{PF}_{6}$ below $T_{\mathrm{SDW}}$. The electronic CDW could also trigger "phase phonons" which should be observable in reflectance measurements. These latter effects apparently have been detected below $T_{\mathrm{SDW}}$ in the parent (TMTSF) ${ }_{2} \mathrm{SbF}_{6}$ compound [91]. However there are very few optical studies performed below $T_{\mathrm{SDW}}$ in (TMTSF) ${ }_{2} \mathrm{X}$ 's to assess this statement.

The coupling between the $2 \mathrm{k}_{\mathrm{F}}$ SDW and the $4 \mathrm{k}_{\mathrm{F}}$ CDW could be achieved by a "magneto-elastic" effect similar to the one considered in (TMTTF $)_{2} \mathrm{Br}$. By analogy with the findings of Annex A.1, this coupling should lead to an increase of hole concentration on the site having the larger magnetization. 
However as the intensity of the $4 \mathrm{k}_{\mathrm{F}} \mathrm{CDW}$ reflections of (TMTSF) $)_{2} \mathrm{PF}_{6}$ is much weaker than the one of $(\mathrm{TMTTF})_{2} \mathrm{Br}$, the lattice distortion which eventually accompanies the $4 \mathrm{k}_{\mathrm{F}} \mathrm{CDW}$ of (TMTSF) ${ }_{2} \mathrm{PF}_{6}$ must be much smaller than the $4 \mathrm{k}_{\mathrm{F}}$ distortion of (TMTTF $)_{2} \mathrm{Br}$. This difference could be explained by the incommensurate nature of the modulation (leading to the loss of umklapp effects) and the smaller amplitude of modulation [a reduction of the magnetization by a factor $1.75\left(0.08 \mu_{\mathrm{B}}\right.$ for $(\mathrm{TMTSF})_{2} \mathrm{PF}_{6}$ versus $0.14 \mu_{\mathrm{B}}$ for (TMTTF) ${ }_{2} \mathrm{Br}$ [71]) reduces, according to (A.2), the amplitude of the distortion by a factor 3 and the $4 \mathrm{k}_{\mathrm{F}}$ reflection intensity by a factor 9$]$. Whatever its microscopic origin, the coupling between the $2 \mathrm{k}_{\mathrm{F}}$ SDW and the lattice remains appreciable in the density wave ground state because optical studies [92] show that the SDW condensate presents a large dynamical mass enhancement, which is however several times smaller than the mass enhancement of a CDW condensate.

\section{The Charge Ordering Transition in the Fabre Salts}

\subsection{Basic Features: The Historical Scenario}

The first indication of the now well established symmetry breaking $\mathrm{CO}$ phase transition was revealed by anomalies in conductivity measurements performed in the (TMTTF) ${ }_{2} \mathrm{X}$ salts with $\mathrm{X}=\mathrm{PF}_{6}$, $\mathrm{AsF}_{6}$ and $\mathrm{SbF}_{6}$ and their solid solutions [93]. The most striking finding was the observation of a MI transition at $T_{\mathrm{CO}}=154 \mathrm{~K}$ in the $\mathrm{SbF}_{6}$ salt, recalling the one previously observed at $160 \mathrm{~K}$ in the SCN salt [66]. Although this transition was correctly interpreted as due to a $4 \mathrm{k}_{\mathrm{F}}$ electronic localization phenomenon, no lattice symmetry breaking could be detected at $T_{\mathrm{CO}}$ in this preliminary study, in the difference of the SCN salt [94]. For this reason this transition was labeled "structureless" in later studies [95] which also revealed an anomaly at $T_{\mathrm{CO}}$ in the thermal dependence of the thermo-power (possibly due to a gap opening) of these salts and similar anomalies in the $\mathrm{ReO}_{4}$ salt well above its $\mathrm{AO}$ transition. During the following year two important, but often ignored studies, revealed a dielectric divergence [81] and a few percent Young modulus softening [96] at $T_{\mathrm{CO}}$. The symmetry breaking consisting of a loss of all the inversion centers at $T_{\mathrm{CO}}$ was only revealed 15 years later by the NMR observation of a charge differentiation between the two TMTTF molecules of the unit cell [97] providing the first evidence of the occurrence of a charge disproportion below $T_{\mathrm{CO}}$. Charge disproportion between molecules was soon confirmed by the observation of split intra-molecular vibration modes below $T_{\mathrm{CO}}[98,99]$. This charge disproportion together with the incipient stack dimerization leads to a stack dielectric polarization and, as there is no cell doubling (at the difference of the SCN salt), to the establishment of electronic ferroelectricity. This was sustained by accurate measurements of the dielectric divergence at $T_{\mathrm{CO}}[100]$.

Although it was suspected in these first works that the anions should control the CO transition [93] or even should be displaced with respect to donors at $T_{\mathrm{CO}}$ [38], several attempts to find a structural modification at $T_{\mathrm{CO}}$ were unsuccessful [7,101-103]. The reason, found recently [104], is that minute irradiation defects created by X-ray beams in laboratory diffraction conditions kill the CO. It was only recently that the finding [24] of a lattice thermal expansion anomaly at $T_{\mathrm{CO}}$ provides convincing evidences of a structural counterpart at the electronic ferroelectricity. Then evidence of a tiny structural modification was found from the detection of weak $(<15 \%)$ variations at $T_{\mathrm{CO}}$ of the intensity of several main Bragg reflections using neutron diffraction on $(\mathrm{TMTTF})_{2} \mathrm{PF}_{6}$ deuterated powders $[21,105]$. These 
quite small structural modifications explain why earlier attempts to solve the (TMTTF) ${ }_{2} \mathrm{PF}_{6}$ and $\mathrm{AsF}_{6}$ structures on hydrogenated crystals in the P1 space group from conventional neutron scattering data collection at $4 \mathrm{~K}$ were unsuccessful [106]. Recently the low temperature non centro-symmetric P1 structure of $(\mathrm{TMTTF})_{2} \mathrm{PF}_{6}$ has been assessed using high energy X-ray beam from synchrotron radiation [107]. The main result of this study was the finding of intra-molecular deformations associated to charge disproportion. However no sizeable change of the F-S contact distance could be detected at $T_{\mathrm{CO}}$.

\subsection{Structural Ingredients of the CO Transition}

From a general point of view, three main structural features are expected at the CO transition of the Fabre salts [108]:

- an internal deformation of the TMTTF molecules following their charge occupancy,

- a deformation of the methyl group cavities associated to the loss of inversion symmetry,

- a shift of the anions from the inversion centers.

The first feature, expected from the splitting of intra-molecular mode frequencies at $T_{\mathrm{CO}}[98,99,109]$, has been recently detected [107]. Although the differentiation of the two molecules of the unit cell already suppresses the inversions centers, a deformation of the methyl group cavities and/or a shift of the anions should also contribute to stabilize the non centro-symmetric structure. In this case both the polarization of the methyl groups and/or the shift of the anions provide the efficient couplings with the $\pi$ cloud $[21,110]$ necessary to stabilize the CO ground state [111].

Figure 8. $T_{\mathrm{CO}}$ in function of the anion (labeled by its mass $\left.\mathrm{M}_{\mathrm{X}}\right)$ in hydrogenated $\left(\mathrm{H}_{12}\right)$ and deuterated $\left(\mathrm{D}_{12}\right)(\mathrm{TMTTF})_{2} \mathrm{X}$ (adapted from [112]). The continuous lines connect $T_{\mathrm{CO}}$ of $\mathrm{H}_{12}$ and $\mathrm{D}_{12}$ salts with anions of same symmetry.

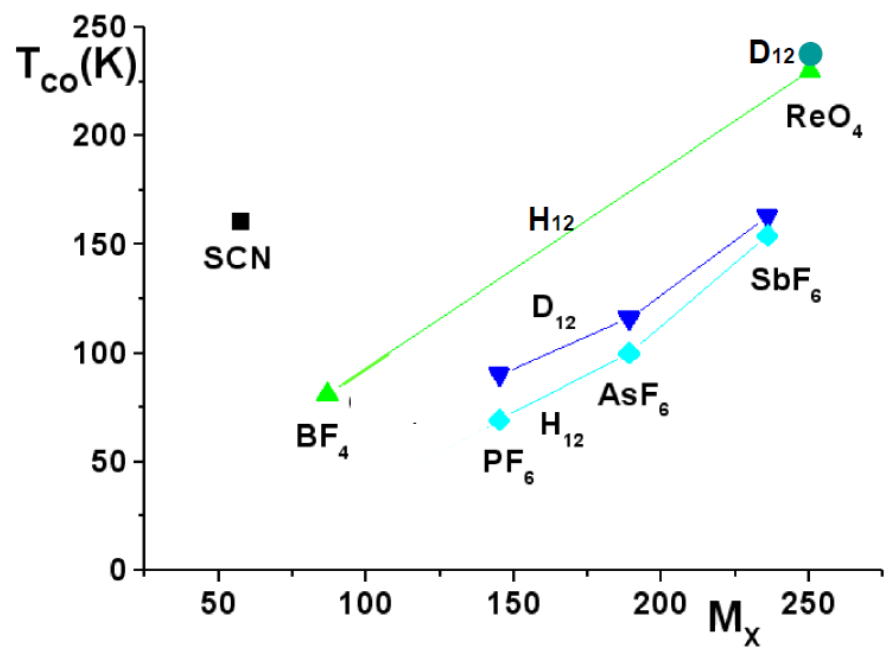

The influence of the anions in the CO process is assessed by the observation that:

- $\quad T_{\mathrm{CO}}$ strongly varies with the volume, shape and polarizability of the anion (see Figure 8) [112],

- $\quad T_{\mathrm{CO}}$ increases when the anion $-\mathrm{S}$ contact distance is shortened [93,113]. 
Figure 9 shows two different processes where the shift of the anion could lead to a disproportion of charge on the TMTTF. In Process 2 of Figure 9, the anion moves towards the $S$ of a TMTTF where the shortening of the anion $-\mathrm{S}$ contact distance below $T_{\mathrm{CO}}$ enhances directly the $\pi$ hole density of the TMTTF in the vicinity of the anion. In Process 1 of Figure 9, the anion moves inside its methyl group cavity and deforms it. This deformation polarizes the H-bond network. The H-bond polarization induces a displacement of charge in the $\sigma$ bonds connected to the H-bonds. This shift of $\sigma$ electrons towards the center of the TMTTF stabilizes the excess of $\pi$ holes [21].

Figure 9. Schematic illustration of the two types of anion shifts. Process 1 (in red): displacement of the anion inside the methyl group cavity which polarizes the H-bond network and the $\sigma$ electron skeleton of the TMTTF. Process 2 (in green): shift of the anion X towards the $\mathrm{Se} / \mathrm{S}$ of a donor $\mathrm{D}$ establishing a short X-D contact distance. The modulation of the density of $\pi$ holes is indicated for Process 1 .

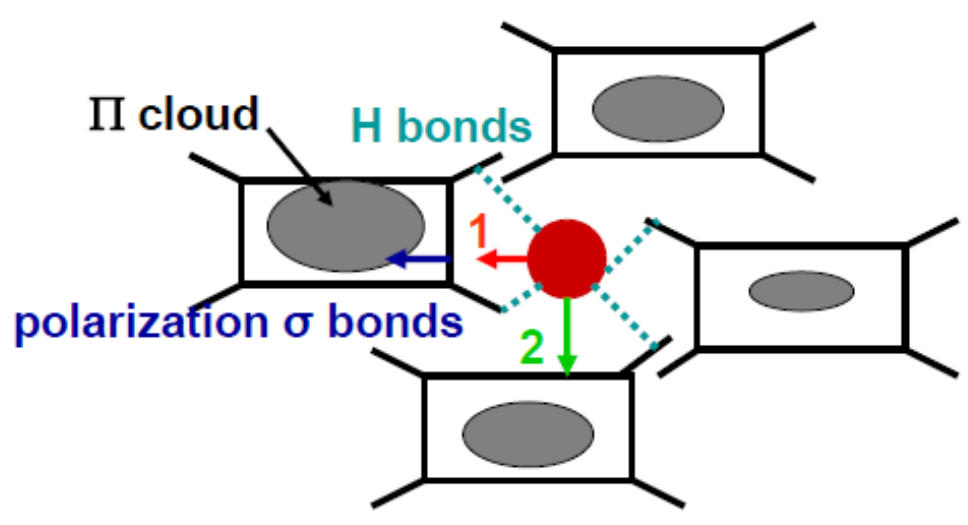

\section{1: $\mathrm{X}$ polarizes $\mathrm{H}$ bonds/methyl group cavity 2: X-D short contact}

Both direct process 2 and indirect process 1 stabilize an excess of $\pi$ hole on the TMTTF towards which the anion moves. However the excess of hole is not localized on the same molecule because the direction of displacement of the anion is different in the two processes. However as the recent structural refinement [107] does not provide evidence of a sizeable change of the anion -S short contact distance below $T_{\mathrm{CO}}$ in the $\mathrm{PF}_{6}$ salt, the stabilization of the $\mathrm{CO}$ pattern probably occurs via the deformation of the methyl group cavity in Process 1 . Such an assertion is sustained by the following observations:

- $\quad T_{\mathrm{CO}}$ increases when, with the larger size (and polarizability) of octahedral and tetrahedral anions, the contact between the anion and the periphery of the cavity delimited by the methyl groups is strengthened,

- $\quad T_{\mathrm{CO}}$ is enhanced by deuteration of the methyl groups of TMTTF [112,114] (the relative increase of $T_{\mathrm{CO}}$ reaches $30 \%$ in the $\mathrm{PF}_{6}$ salt),

- CO is rapidly destroyed by X-ray irradiation damages [104] which particularly affect terminal methyl groups of the donor (in a general manner irradiation effects are especially strong in salts built with the TMTTF and TMTSF methyl-based donors [115]). 
- Far infrared measurements show there is a modification of the coupling between the methyl groups and the anions at the CO transition [109].

Interactions via the methyl groups could also explain why strong $T_{\mathrm{CO}}$ lattice parameter anomalies are observed in the $\left(\mathrm{b}^{\prime}, \mathrm{c}^{*}\right)$ plane containing the H-bond network (see Figure 1); the strongest anomaly being along $c^{*}$ [24]. In addition the easy squeezing of the soft methyl group cavity under pressure explains simply, via the blockade of the anion in its cavity, the rapid decrease of $\mathrm{T}_{\mathrm{CO}}$ under pressure [116]. Until now there is no direct evidence of the deformation of the methyl group cavity at the $\mathrm{CO}$ transition. This deformation should be better revealed by neutron diffraction more sensitive to $\mathrm{H}$ or D positions.

An eventual shift of the anion in the $\mathrm{CO}$ phase does not mean that the anion orientation disorder is completely removed at $T_{\mathrm{CO}}$ in the TMTTF salts. ${ }^{19} \mathrm{~F}$ NMR studies of $(\mathrm{TMTTF})_{2} \mathrm{SbF}_{6}$ show the contrary [27,28]. Evidence of the decoupling between $\mathrm{CO}$ and orientation order of the anion is provided by the observation of successive $\mathrm{CO}$ and $\mathrm{AO}$ transitions in the $\mathrm{ReO}_{4}\left(T_{\mathrm{CO}}=230 \mathrm{~K}\right.$ and $\left.T_{\mathrm{AO}}=154 \mathrm{~K}\right)$ and $\mathrm{BF}_{4}\left(T_{\mathrm{CO}}=83 \mathrm{~K}\right.$ and $\left.T_{\mathrm{AO}}=40 \mathrm{~K}\right)$ salts [117].

Process 1 in Figure 9 which achieves an anion shift inside the methyl group cavity is structurally different from Process 2 which achieves an anion shift towards the $S$ of the donor as found at the (1/2, $1 / 2,1 / 2$ ) AO transition (see Section 7.2). It is thus possible that these two types of nearly perpendicular anion displacements could be successively active in the $\mathrm{CO}$ and $(1 / 2,1 / 2,1 / 2) \mathrm{AO}$ transitions of the TMTTF salts with the $\mathrm{ReO}_{4}$ and $\mathrm{BF}_{4}$ tetrahedral anions. This decoupling allows to simply understand that the $(1 / 2,1 / 2,1 / 2) \mathrm{AO}$ transition, which does not substantially activate the H-bond network, is less sensitive to deuteration [114] and irradiation defects [118].

In this picture TMTTF salts incorporating small anions, such as $\mathrm{ClO}_{4}$ and $\mathrm{NO}_{3}$ which do not establish important interactions with the methyl groups [14,110] should not exhibit the CO transition. This seems to be the case for the $\mathrm{ClO}_{4}$ salt [113]. In the opposite situation where an anion perfectly fits the methyl group cavity there is no room for an anion shift inside this cavity and thus no $\mathrm{CO}$ transition is expected. This could be the case of the $\mathrm{Br}$ salt. This better fit, which also occurs under pressure with the squeezing of the methyl group cavities, can be taken as responsible of the rapid drop of $T_{\mathrm{CO}}$, as observed for example in pressurized $\mathrm{AsF}_{6}$ salt [116].

In this framework the $(0,1 / 2,1 / 2)$ anti-ferroelectric $\mathrm{CO}$ transition of (TMTTF) ${ }_{2} \mathrm{SCN}$, which coincides with the AO transition of the SCN [94], is singular. In this salt the small SCN anion, which has no real linkage with the methyl groups [110], can only strengthen its contacts with one donor out of two via the AO shift [103]. In this respect the wave vector of the AO superstructure of (TMTTF $)_{2} \mathrm{SCN}$, which simultaneously stabilizes the $\mathrm{CO}$, is different from the wave vectors stabilized at the others AO and CO transitions (see Section 7). The AO/CO transition of the SCN salt which does not directly involve the methyl groups is consistently weakly sensitive to X-ray irradiation damage.

Because of the involvement of the fragile methyl groups in the stabilization of the CO pattern, the ferroelectric ground state is subject to defects. Defects break the long range ferroelectric order into domains forming local clusters of polarization. This leads to a frequency dependant dielectric permittivity which does not really diverge at a well defined $T_{\mathrm{CO}}$. Such features, recalling those of dielectric relaxors, are found in (TMTTF $)_{2} \mathrm{PF}_{6}$ at ambient pressure and in (TMTTF $)_{2} \mathrm{SbF}_{6}$ under 
pressure near its transformation to a (local) SP ground state [117]. The domain walls nucleated by these defects are described by charge soliton excitations of the CO ground state [119].

Electronic ferroelectricity [100,119] occurs in others organic systems [120]. Among them let us mention the $2 \mathrm{D}$ organic salt $\alpha-(\mathrm{BEDT}-\mathrm{TTF})_{2} \mathrm{I}_{3}$ where ferroelectricity appears at the $135 \mathrm{~K} \mathrm{MI}$ transition [121]. The interesting aspect of this phase transition is that, analogously to TMTTF salts, the charge rearrangement on type A BEDT-TTF molecules (called CO for this reason) is triggered by the deformation of the $I_{3}$ sublattice which thus modifies of the H-bond network between $I_{3}$ and the ethylene groups of the BEDT-TTF [122]. This mechanism, similarly to Process 1 in Figure 9, modulates the density of $\pi$ holes on the BEDT-TTF.

\subsection{The Underlying CO Instability}

The CO transition observed in the Fabre salts is just a manifestation of the general tendency of organic conductors to form a Wigner lattice of localized charges because of the presence of long range intermolecular Coulomb repulsions (for a recent review see [73]). As the lattice is soft such an electronic instability is coupled to the lattice where it drives a $4 \mathrm{k}_{\mathrm{F}} \mathrm{CDW}$ which also, in presence of an electron-phonon coupling with the acoustic modes, leads to the formation of a $4 \mathrm{k}_{\mathrm{F}} \mathrm{BOW}[123,36]$. However in quarter filled band systems, one has to distinguish between two different types of charge localization phenomena [73,76]:

- charge localization on the bonds, stabilizes a $4 \mathrm{k}_{\mathrm{F}}$ BOW or Dimer Mott (DM) ground state,

- charge localization on the sites, stabilizes a $4 \mathrm{k}_{\mathrm{F}} \mathrm{CDW}$ or CO ground state.

For uniform quarter filled band systems, these two ground states have different inversion symmetry. However the TMTTF salts are more complex because with a stack being already dimerized (see Section 2.2) the divergence of the $4 \mathrm{k}_{\mathrm{F}}$ BOW instability is killed (the static dimerization itself gives rise to a charge localization [12] "visualized" by the development of an activated conductivity below $T \rho \sim$ $200 \mathrm{~K}$ higher than $T_{\mathrm{CO}}$ ). Thus the only symmetry breaking instability remaining in the dimerized salts is the $\mathrm{CO}$ if the stacks are not too strongly dimerized [73,119].

Ferroelectric $\mathrm{CO}$ is not announced by easily detectable pre-transitional structural fluctuations (the pretransitional structural fluctuations at the anti-ferroelectic $\mathrm{CO}$ transition of (TMTTF) ${ }_{2} \mathrm{SCN}$ are also barely detectable). Also the ferroelectric instability associated to $\mathrm{CO}$ appears to be mean-field because the dielectric susceptibility divergence follows a Curie Weiss behavior on a large ( $30 \mathrm{~K}$ ) temperature range above $T_{\mathrm{CO}}[100,117]$. This means that $\mathrm{CO}$ in the TMTTF's is mostly announced by a regime of 3D fluctuations which isotropy reflects the importance of the inter-chain Coulomb coupling. In contrast, regular (i.e., non dimerized) quarter filled systems such as (DIDCNQI) $2 \mathrm{Ag}$ [124] or (o-DMTTF) $)_{2}$ [125] exhibit a sizeable regime of $1 \mathrm{D} 4 \mathrm{k}_{\mathrm{F}}$ BOW fluctuations above the "CO" transition which in fact presents a mixed CO/DM character. 


\section{The Spin-Peierls Transition in the Fabre Salts}

\subsection{Basic Features}

The SP transition of (TMTTF $)_{2} \mathrm{PF}_{6}$ is characterized by the appearance below $T_{\mathrm{SP}} \sim 17 \mathrm{~K}$ of superlattice reflections of very weak intensity at the $(1 / 2,1 / 2, ?)$ reduced reciprocal position according to earlier X-ray investigations [40] (see Figure 10). The third component $1 / 2 \mathrm{c}^{*}$ has been determined by a recent neutron scattering investigation [68].

Figure 10. $\mathrm{X}$-ray diffuse scattering pattern from (TMTTF) ${ }_{2} \mathrm{PF}_{6}$ at $10 \mathrm{~K}$ in its $\mathrm{SP}$ ground state showing inside the blue circles very weak $(1 / 2,1 / 2, ?)$ superlattice spots. The $\left(a^{*}, b^{*}\right)$ unit cell is shown (original data of the study reported in [40]).

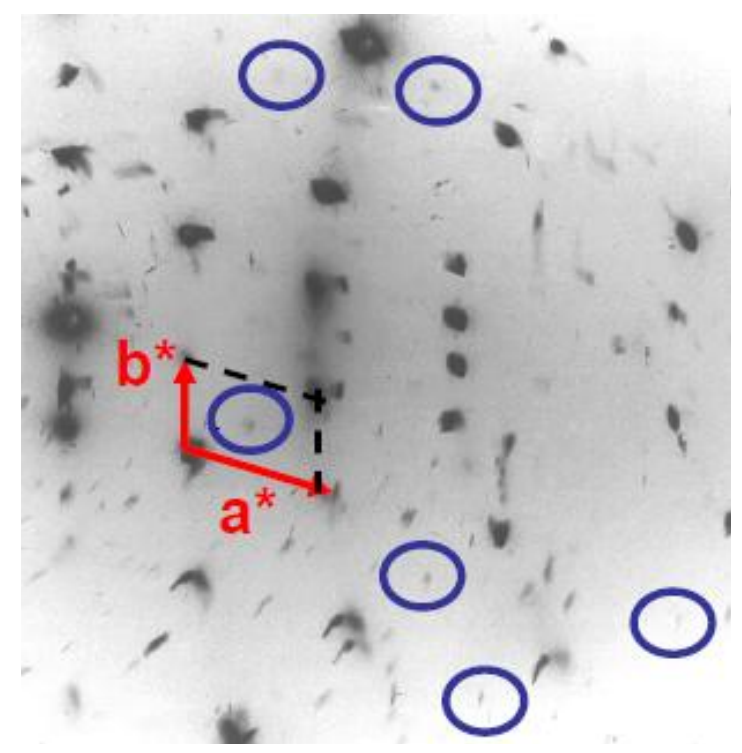

At ambient pressure the SP transition of the TMTTF's occurs in the CO phase. The SP order is rapidly destroyed by X-ray irradiation as for the $\mathrm{CO}$ transition. This irradiation sensitivity explains that recent structural studies of the SP order have been performed with neutron scattering. The structure of the SP ground state has not yet been determined, but it should present some analogies with the (1/2, $1 / 2,1 / 2)$ AO superstructure $[58,59]$. Thus it is expected that the stack tetramerization, corresponding to a dimerization of stack of dimers, each dimer bearing a spin $1 / 2$, will be accompanied by synchronized shift of the anions towards donors [68]. The involvement of the anions in the SP instability is assessed by the observation of a $T_{\mathrm{SP}}$ critical divergence of the ${ }^{75} \mathrm{As} \mathrm{NMR}_{1}{ }^{-1}$ in $(\mathrm{TMTTF})_{2} \mathrm{AsF}_{6}[126,127]$. In this process the anion shift should tune the interchain coupling between the individual stack tetramerization, as discussed in Section 3.1.2. The 3D inter-chain coupling regime should occur in the near vicinity of $T_{\mathrm{SP}}$ because the observation of precursor X-ray diffuse scattering lines on a large temperature range above $T_{\mathrm{SP}}$ shows that the SP instability is basically $1 \mathrm{D}$ (see Section 3.3).

The temperature $T_{\mathrm{SP}}$ at which the $(1 / 2,1 / 2,1 / 2)$ superlattice reflections are observed coincides with the temperature at which a singlet gap develops in the spin susceptibility [112]. At $T_{\mathrm{SP}}$ lattice expansion measurements exhibit a lambda type anomaly which coincides with the specific heat anomaly [128]. The value of the singlet-triplet gap in controversial in the literature which mixes 
calculated values, using approximate theories [129,130], and measured quantities [74,112]. These last measurements have been confirmed by a direct determination, using inelastic neutron scattering, of the SP gap in the spin excitation spectrum [131]. The SP wave vector is the same in the TMTTF salts and in the isostructural $\mathrm{BCPTTF}$ salts $[7,70]$. BCPTTF $)_{2} \mathrm{PF}_{6}$ and $\mathrm{AsF}_{6}$, which does not undergo a $\mathrm{CO}$ transition, have a twice higher $T_{\mathrm{SP}}$ than (TMTTF) ${ }_{2} \mathrm{PF}_{6}$ and $\mathrm{AsF}_{6}$.

The SP transition is very sensitive to the magnetic field, $\mathrm{H}$, as in others SP compounds such as MEM-(TCNQ) 2 and $\mathrm{CuGeO}_{3}$. In (TMTTF) $)_{2} \mathrm{PF}_{6} T_{\mathrm{SP}}$ decreases as $\mathrm{H}^{2}$ [132] and above 19T this salt undergoes a phase transition to an incommensurate soliton-like structure [133,134].

\subsection{Competition with the Charge Ordering}

NMR measurements show that there is coexistence between $\mathrm{CO}$ and SP pairing in $(\mathrm{TMTTF})_{2} \mathrm{PF}_{6}$ and $\mathrm{AsF}_{6}$. However the observation of an opposite variation of $T_{\mathrm{CO}}$ and $T_{\mathrm{SP}}$ under pressure shows that there is repulsion between the order parameters associated at the CO and SP transitions [116]. In this context, the real part of the microwave dielectric function, $\varepsilon$, which increases by $\sim 10 \%$ in the SP phase, shows that the gap of charge (i.e., CO order parameter) decreases at the SP transition [132]. This competition is analyzed in Annex A.3 in the framework of the Landau theory. In particular Landau development predicts the behavior of $T_{\mathrm{SP}}$ in function of $T_{\mathrm{CO}}$. This dependence, obtained with the restrictions outlined in Annex A3 and shown in Figure 11, accounts for the experimental results.

Figure 11. Spin-Peierls critical temperature, $T_{\mathrm{SP}}$, of (TMTTF $)_{2} \mathrm{X}$ versus $\sqrt{ }\left(T_{\mathrm{CO}}-T_{\mathrm{SP}}\right)$, as given by the expression (A12). $T_{\mathrm{SP}}{ }^{0}$ of (BCPTTF) $)_{2} \mathrm{X}$, which does not undergo a $\mathrm{CO}$, is also given, as well as $\sqrt{ }\left(T_{\mathrm{CO}}\right)$ for the $\mathrm{SbF}_{6}$ and $\mathrm{SCN}$ salts having no SP ground state. The spin-liquid phase occurring for $T_{\mathrm{SP}}<5 \mathrm{~K}$ (see text) is indicated.

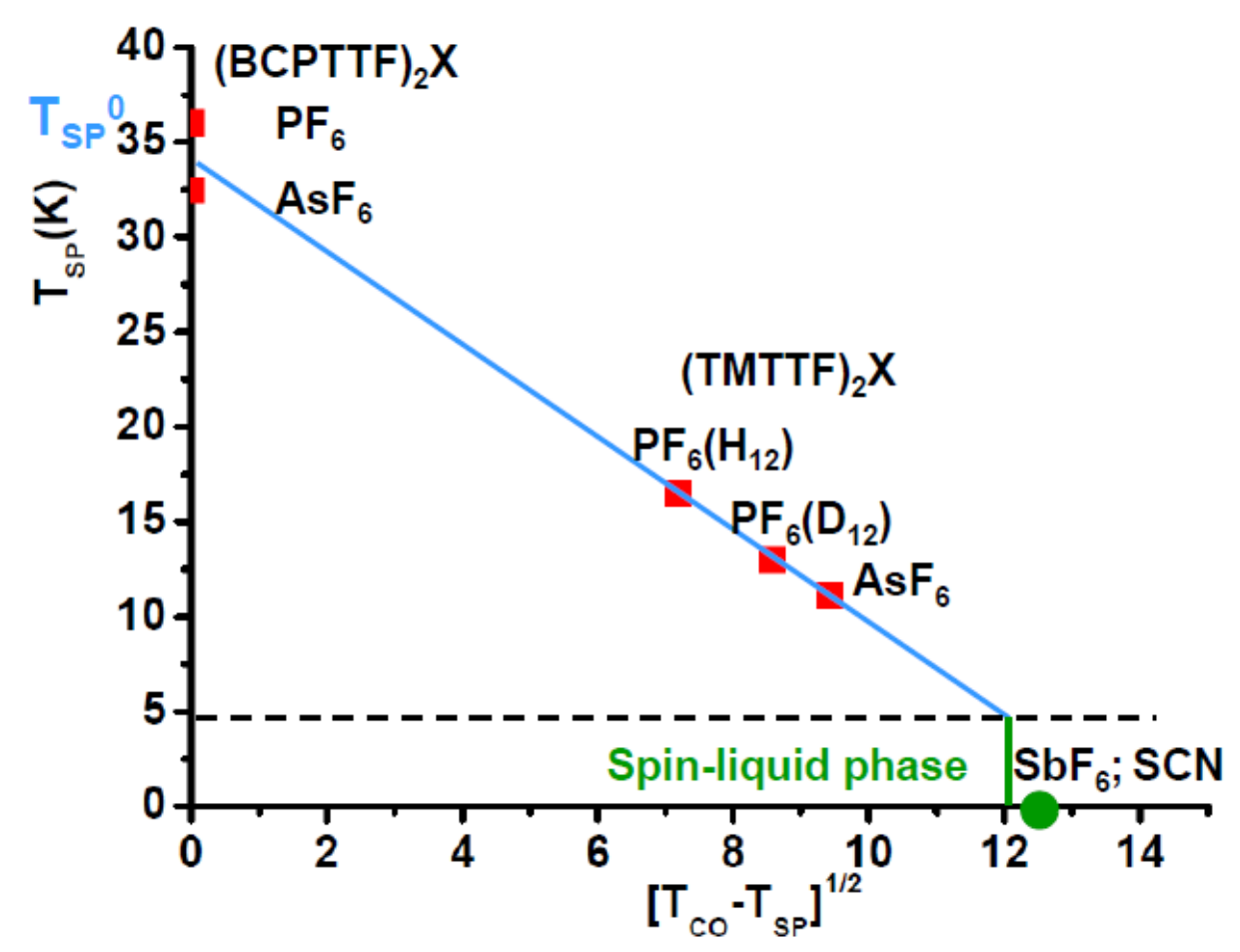


Figure 11 shows that $T_{\mathrm{SP}}$ of $(\mathrm{BCPTTF})_{2} \mathrm{PF}_{6}$ and $\mathrm{AsF}_{6}$ is consistent with the absence of $\mathrm{CO}$ (in that case the SP transition occurs at $T_{\mathrm{SP}}{ }^{0}$ defined Annex A3). Figure 11 shows also that the SP transition vanishes for $T_{\mathrm{CO}} \sim 190 \mathrm{~K}$ or more likely for $\sim 150 \mathrm{~K}$ if one assumes that for $T_{\mathrm{SP}}<5 \mathrm{~K}$ the spin-liquid phase is stabilized (see below). $150 \mathrm{~K}$ is close to $T_{\mathrm{CO}}$ of (TMTTF) ${ }_{2} \mathrm{SbF}_{6}$ and $\mathrm{SCN}$ which do not exhibit a SP ground state.

To describe completely the SP phase diagram of the TMTTF salts, one has to consider explicitly the non-adiabatic effect of the phonon field (see Section 3.3). These effects have already been discussed in [73] with the main conclusions being:

- SP transition does not occur in (TMTTF $)_{2} \mathrm{SbF}_{6}$ and $\mathrm{SCN}$ because the spin-phonon coupling is smaller than the zero-point lattice fluctuations (this condition corresponds to $T_{\mathrm{SP}}{ }^{\mathrm{MF}}<\Omega / 4 \sim 15 \mathrm{~K}$, or $T_{\mathrm{SP}}<5 \mathrm{~K}$ because in the $\mathrm{PF}_{6}$ and $\mathrm{AsF}_{6}$ salts $\left.T_{\mathrm{SP}} \sim T_{\mathrm{SP}}{ }^{\mathrm{MF}} / 3\right)$. These salts are thus located in the spin-liquid phase outlined in Figure 11. In this phase the inter-chain exchange coupling stabilizes a low temperature $\left(T_{\mathrm{N}} \sim 7 \mathrm{~K}\right) \mathrm{AF}$ ground state.

- $\quad$ SP transitions of $(\mathrm{BCPTTF})_{2} \mathrm{PF}_{6}$ and $\mathrm{AsF}_{6}$ occur in the adiabatic limit.

- SP transitions of $(\mathrm{TMTTF})_{2} \mathrm{PF}_{6}$ and $\mathrm{AsF}_{6}$ are located between the adiabatic limit and the spin-liquid phase, close to the crossover line between the adiabatic and anti-adiabatic regimes.

The microscopic origin of the competition between $\mathrm{CO}$ and SP instabilities relies on the fact that $\mathrm{CO}$, which localizes the holes every second neighbor molecules, establishes also a reduced AF exchange integral, J, between the spin of the holes. With a spin-phonon coupling following the decrease of $\mathrm{J}, T_{\mathrm{SP}}{ }^{\mathrm{MF}}$ (i.e. the SP instability) decreases [73].

The weakening of the SP instability by the $\mathrm{CO}$ is just the manifestation of the repulsion existing between $4 k_{\mathrm{F}} \mathrm{CDW}$ (or $\mathrm{CO}$ ) and $2 \mathrm{k}_{\mathrm{F}}$ BOW (or SP) instabilities in quarter filled band systems: the next neighbour Coulomb repulsion $\mathrm{V}_{1}$ which promotes $\mathrm{CO}$ in quarter filled band systems kills in the same time the divergence of the $2 \mathrm{k}_{\mathrm{F}}$ BOW response function [135,136]. In other words CO induces hetero-polar charge configurations which destabilize the modulation of bond distances in the $2 k_{\mathrm{F}}$ Peierls mechanism. This was clearly shown in the study of the $\left[(\mathrm{TMTSF})_{1-} x(\mathrm{TMTTF}) x\right]_{2} \mathrm{ReO}_{4}$ solid solution $[137,138]$. For $x=0.5$ the alternate stacking of TMTSF and TMTTF induces a chemical CO. In its presence it is observed a sizeable decrease of $\mathrm{T}_{\mathrm{AO}}$ of the $(1 / 2,1 / 2,1 / 2) \mathrm{AO}$ transition which involves the formation of $2 \mathrm{k}_{\mathrm{F}} \mathrm{BOW}$ on the organic stacks (see Section 7.2).

\subsection{The Vanishing of the SP Phase under Pressure}

$T_{\mathrm{SP}}$ increases when $T_{\mathrm{CO}}$ decreases under pressure [116]. At higher pressure when the $\mathrm{CO}$ vanishes instead of reaching $T_{\mathrm{SP}}{ }^{0}$ at a value comparable to $T_{\mathrm{SP}}$ measured in the BCPTTF's, $T_{\mathrm{SP}}$ further decreases. In the $\mathrm{PF}_{6}$ salts $T_{\mathrm{SP}}$ even exhibits a singular depression around $\mathrm{Pc} \sim 9$ kbar before entering the $\mathrm{AF}$ phase [139]. This behavior suggests the existence of a quantum critical point where the normal phase is further stabilized around Pc.

A possible explanation could be, that with TMTTF salts being close to the crossover boundary between the adiabatic and anti-adiabatic regime, the hardening of the phonon mode under pressure will shift the SP instability to the anti-adiabatic regime followed by the spin-liquid regime around Pc. In the anti-adiabatic SP regime the enhancement of quantum fluctuations should strongly reduce the 
amplitude of 1D SP gap. In this scenario the suppression of the SP phase should occur through a critical point due to the enhancement of spin-liquid fluctuations. Then above $\mathrm{P}_{c}$ the $3 \mathrm{D} A F$ ground state will be stabilized by the inter-chain exchange coupling. If the succession of ground states is described by the generic phase diagram [52], AF pressurized (TMTTF) ${ }_{2} \mathrm{PF}_{6}$ should be identical to AF ambient pressure $(\mathrm{TMTTF})_{2} \mathrm{Br}$.

In order to assess the development of a pressure induced non-adiabaticity one must prove that the amplitude of the 1D SP gap decreases under pressure as a result of the enhanced quantum fluctuations. However, as it is observed that $T_{\mathrm{SP}}{ }^{\mathrm{MF}}$ (linearly related to the mean field 1D SP gap when the phonon fluctuations are neglected) slightly increases from (TMTTF) $)_{2} \mathrm{PF}_{6}$ to (TMTTF) ${ }_{2} \mathrm{Br}$ (see Figure 5), the opposite situation seems to occur. In addition in order to sustain the non-adiabatic scenario one should have a considerable phonon frequency hardening (by a factor $\sim 4$ ) to stabilize the spin-liquid phase in pressurized (TMTTF) ${ }_{2} \mathrm{PF}_{6}$. This requires a phonon frequency as high as $\Omega>4 T_{\mathrm{SP}}{ }^{\mathrm{MF}}$, which seems unrealistic for an acoustic mode. The difficulty with this scenario is that SP fluctuations are still detected in AF (TMTTF) $)_{2} \mathrm{Br}$, at the difference of (TMTTF) $)_{2} \mathrm{SbF}_{6}$ where it was stated in Section 6.2 that the AF ground state is caused by the enhanced non-adiabaticity of the phonon field which in the same time kills the SP fluctuations.

Another scenario could be that the squeezing of the methyl group cavities under pressure blocks the shift of the $\mathrm{PF}_{6}$, the necessary ingredient setting the interchain coupling between the individual SP stack tetramerization. This is corroborated by a recent determination of the structure of pressurized $(\mathrm{TMTTF})_{2} \mathrm{PF}_{6}$ [140] showing a sizeable contraction of the methyl group cavity. The mechanism of blockade of the anion in its methyl group cavity explains also that only a short range SP order is stabilized in pressurized (TMTTF) ${ }_{2} \mathrm{SbF}_{6}$ [27] when both the $\mathrm{CO}$ and AF phases are suppressed above $0.5 \mathrm{GPa}$.

\section{The Anion Ordering Transitions}

\subsection{Basic Features}

In Fabre and Bechgaard salts incorporating non-centrosymmetric anions, the anion orientation is disordered at RT. However for entropy reasons, the anions order upon cooling. The ordering is achieved at a well defined phase transition showing that the AO leads to a symmetry breaking. The AO transition stabilizes a superstructure which generally doubles the RT lattice periodicity in one or several directions [7]. The AO transition can be of $2^{\text {nd }}[(0,1 / 2,0),(1 / 2,0,0)$ and $(0,1 / 2,1 / 2) A O]$ or 1 st order $[(1 / 2,1 / 2,1 / 2) \mathrm{AO}]$ depending on the strength of the coupling of the AO process at the elastic lattice degrees of freedom The transition not only consists of an orientation ordering of the anion inside its methyl group cavity, which in itself breaks its inversion symmetry, but also of a concomitant deformation of this cavity associated with an anion shift. This shift can be either towards selected methyl groups as in the $(0,1 / 2,0) \mathrm{AO}$ transition of (TMTSF) ${ }_{2} \mathrm{ClO}_{4}$ (direction 1 in Figure 9) [141] or towards the $S$ or Se atom of a given donor as in the $(1 / 2,1 / 2,1 / 2)$ AO transition of (TMTSF) ${ }_{2} \mathrm{ReO}_{4}$ (direction 2 in Figure 9) [58,59]. The deformation of the methyl group cavities can be achieved by a cooperative PLD of the organic stacks. In the case of the $(1 / 2,1 / 2,1 / 2)$ AO transition this corresponds to a $2 \mathrm{k}_{\mathrm{F}}$ BOW distortion of the stacks. In (TMTSF) ${ }_{2} \mathrm{ReO}_{4}$ the $2 \mathrm{k}_{\mathrm{F}}$ PLD leads to a MI 
transition [142]. Even if the coupling of the PLD with the 1D electron gas provides the necessary energy gain to open a gap at the Fermi level, the $(1 / 2,1 / 2,1 / 2)$ AO transition is not achieved by a standard Peierls instability because the associated pretransitional fluctuations are 3D and not 1D [143]. This means that the anions located in between the stacks trigger by their ordering the $2 \mathrm{k}_{\mathrm{F}}$ stack deformation while providing the necessary inter-chain coupling to stabilize the AO insulating ground state. This differs of a conventional Peierls transition where the driving force is due to the individual stack instability towards the formation of a $2 \mathrm{k}_{\mathrm{F}}$ PLD. Indeed the electron-anion coupling displayed in Figure 9 plays in the AO transition the role played by the intra-stack electron-phonon coupling in the Peierls transition.

These few examples, as well as the case of (TMTTF) ${ }_{2} \mathrm{SCN}$ already considered Section 5.2, show that important changes of the electronic properties generally accompany the AO transition. AO transitions lead to substantial modifications of the electronic structure [144,145] and of the electronic ground states [146,147] of the Bechgaard and Fabre salts. These structural-electronic coupled properties will be revisited below in light of the most recent findings.

\subsection{The (1/2, 1/2, 1/2) AO Transition of Tetrahedral Anions}

Among salts undergoing an $(1 / 2,1 / 2,1 / 2)$ AO transition, several cases must be distinguished:

- TMTSF salts where the AO transition coincides with a MI transition.

- TMTTF salts where $4 \mathrm{k}_{\mathrm{F}}$ charge localization takes place above the AO transition.

- Salts with dipolar anions which incorporate additional degrees of freedom.

\subsubsection{TMTSF Salts}

$\mathrm{ReO}_{4}$ and $\mathrm{BF}_{4}$ salts undergo 1 st order $\mathrm{AO} / \mathrm{MI}$ transitions, at $T_{\mathrm{AO}}=176 \mathrm{~K}$ and $36 \mathrm{~K}$ respectively, which open a sizeable energy gap of $\sim 0.2 \mathrm{eV}$ [142]. These MI transitions cannot be classified as standard Peierls transitions because these latter are 2nd order transitions and open much smaller energy gaps. For these reasons it has been suggested [148] that these AO transitions should be understood in the framework of a strong-coupling theory where the anion shift towards the Se of the closest TMTSF $[58,59,149]$ is the essential ingredient. In this picture the anion shift, associated to its orientation ordering, localizes a $\mathrm{p}_{\pi}$ hole on the molecule towards which the anion establishes a short contact. In this mechanism the magnitude of the energy gap should reflect the staggered Hartree anion potential experienced by the $\mathrm{p}_{\pi}$ holes. However structural refinements of (TMTSF)2 $\mathrm{ReO}_{4}$ [59] and $\mathrm{BF}_{4}$ [149] superstructures do not exhibit an appreciable internal deformation of the TMTSF revealing the formation of a sizeable $2 \mathrm{k}_{\mathrm{F}}$ CDW induced by the direct Coulomb coupling with the anions. Structural refinements [58,59] show that the $2 \mathrm{k}_{\mathrm{F}}$ BOW stack distortion consists mainly in a displacement wave of an amplitude $\sim 0.07 \AA$ (essentially directed along c) of dimers, composed of the two inequivalent donors below $T_{\mathrm{AO}}$, which alternates in stack direction by inversion symmetry in order to set the $2 \mathrm{k}_{\mathrm{F}}$ periodicity. However tight binding band calculations $[149,150]$ considering only the $2 \mathrm{k}_{\mathrm{F}}$ stack distortion cannot account for the entire value of the energy gap. Thus it should be interesting to perform a DFT calculation of the electronic structure which fully incorporates the shifted and ordered anions. 
The AO transition of the $\mathrm{ReO}_{4}$ salt occurs in the temperature range above $T_{\mathrm{X}}$ where the warping of the FS is not relevant, but this is probably not the case for the $\mathrm{BF}_{4}$ salt. Under pressure it is observed that the MI transition of the $\mathrm{ReO}_{4}$ salt disappears [151] when the superstructure periodicity changes from $(1 / 2,1 / 2,1 / 2)$, to $(0,1 / 2,1 / 2)$ through a $1^{\text {st }}$ order phase transition [152]. When, with the setting of the $(0,1 / 2,1 / 2)$ superstructure, the metallic state is restored pressurized (TMTSF) ${ }_{2} \operatorname{ReO}_{4}$ becomes a superconductor with a $T_{\mathrm{S}}$ as high as $1.5 \mathrm{~K}$ [149]-1.7 $\mathrm{K}$ [153] in the coexistence region of the two AO superstructures. The $(0,1 / 2,1 / 2)$ superstructure, occurring at a $T_{\mathrm{AO}}$ of $240 \mathrm{~K}$ at $1.7 \mathrm{GPa}$, could be stabilized at ambient temperature around $2.5 \mathrm{GPa}$. The squeezing of the methyl group cavities under pressure is certainly responsible for the locking of the orientation of the anions in the $(0,1 / 2,1 / 2)$ superstructure. A refinement of this superstructure would be desirable.

\subsubsection{TMTTF Salts}

Although presenting structural distortions of same nature as in TMTSF salts, the $(1 / 2,1 / 2,1 / 2)$ AO ground state of the TMTTF salts exhibit different electronic properties. At atmospheric pressure, $T_{\mathrm{AO}}$ occurs in the $4 \mathrm{k}_{\mathrm{F}}$ charge localization regime (i.e. charge localization in the TMTTF dimers below $T_{\rho}$ ). $T_{\mathrm{AO}}$ occurs even below the $\mathrm{CO}$ transition $\left(T_{\mathrm{CO}}<T_{\rho}\right)$ in the $\mathrm{ReO}_{4}$ and $\mathrm{BF}_{4}$ salts. $T_{\mathrm{CO}}$ and $T_{\mathrm{AO}}$ are quite decoupled in these salts, which means, as already discussed in Section 5.2, that the anions, remaining disordered below $T_{\mathrm{CO}}$, undergo a new shift towards the $\mathrm{S}$ of the TMTTF when they order at $T_{\mathrm{AO}}$.

Structural refinement below $T_{\mathrm{AO}}[103]$ shows that (TMTTF) ${ }_{2} \mathrm{ReO}_{4}$ exhibits both:

- a sizeable $2 \mathrm{k}_{\mathrm{F}} \mathrm{CDW}$ with an amplitude of 0.25 electrons estimated from the bond lengths of the two inequivalent TMTTF of the dimer, and which must add to a $\mathrm{CO}$ amplitude of 0.17 electrons estimated by NMR between $T_{\mathrm{CO}}$ and $\mathrm{T}_{\mathrm{AO}}$ [154],

- a sizeable $2 \mathrm{k}_{\mathrm{F}}$ BOW achieving a quite large dimerization of the stack of dimers with substantially different inter-dimer distances of $3.9 \AA$ and $3.75 \AA$; the intra-dimer distance being of $3.7 \AA$.

Indeed a significant increase of the activation energy of the conductivity $[113,117]$ and a rapid drop of spin susceptibility $[118,130]$ follow the $2 \mathrm{k}_{\mathrm{F}} \mathrm{AO}$ structural distortion.

The AO transition of (TMTTF) $)_{2} \mathrm{BF}_{4}$ is quantitatively different from the $\mathrm{AO}$ transition of the $\mathrm{ReO}_{4}$ salt because there is no appreciable change of the activation energy of the conductivity $[66,113]$ and only a weak drop of spin susceptibility $[118,130]$ at $T_{\mathrm{AO}}$. This should be due to the presence of a very weak $2 \mathrm{k}_{\mathrm{F}}$ PLD of the stack below $T_{\mathrm{AO}}$. In this picture the slight increase of conductivity observed at $T_{\mathrm{AO}}$ [113] can be explained by an increase of carrier mobility due to the reduction of the electron scattering by the AO. This finding is very surprising because a large gap of charge opens at the AO transition of $(\mathrm{TMTSF})_{2} \mathrm{BF}_{4}$. A possible explanation could be that the high temperature $\mathrm{CO}$ of (TMTTF $)_{2} \mathrm{BF}_{4}$ kills substantially the $2 \mathrm{k}_{\mathrm{F}} \mathrm{BOW} / \mathrm{SP}$ response of the stack at the staggered AO potential (see the end of Section 6.2), leaving the stack nearly unaffected by the AO transition. In this case the $\mathrm{AO}$ transition should consist in a simple ordering of the $\mathrm{BF}_{4}$.

Similar features $[66,113,118,130]$ are observed at the AO transition of $(\mathrm{TMTTF})_{2} \mathrm{ClO}_{4}$ where the structural refinement performed below $T_{\mathrm{AO}}$ [155] shows that only very weak anion shift and stack tetramerization accompany the AO process. The AO transition of (TMTTF) ${ }_{2} \mathrm{ClO}_{4}$ thus corresponds to 
the simple ordering of well decoupled $\mathrm{ClO}_{4}$, a finding in agreement with a measured entropy of transition of $\mathrm{k}_{\mathrm{B}} \ln 2$ per formula unit [66].

\subsubsection{Salts with Dipolar Tetrahedral Anions}

Bechgaard and Fabre salts incorporate also non symmetrical tetrahedral anions such as $\mathrm{PF}_{2} \mathrm{O}_{2}$ and $\mathrm{FSO}_{3}$ which possess an electric dipolar momentum. These anions provide an additional degree of freedom in the structure related to the orientation of their electric dipoles.

$(\mathrm{TMTSF})_{2} \mathrm{PF}_{2} \mathrm{O}_{2}$ exhibits two competitive instabilities at ambient pressure which stabilize after a 2nd order transition the $(1 / 2, \pm 1 / 4,0)$ superstructure followed a few degrees below by a $1^{\text {st }}$ order transition towards the more "conventional" $(1 / 2,1 / 2,1 / 2)$ superstructure [156]. It is not known if the dipole of the anion is ordered in these superstructures. Note that the $(1 / 2, \pm 1 / 4,0)$ superstructure does not fulfill all the conditions (5) minimizing the interchain coupling via the anion shift.

(TMTTF $)_{2} \mathrm{FSO}_{3}$ undergoes a 1 st order transition at $58 \mathrm{~K}$ which stabilizes the $(1 / 2,1 / 2,1 / 2)$ AO superstructure. However at the difference of the (F-1) AO superstructure of $(\mathrm{TMTTF})_{2} \mathrm{ReO}_{4}$, which contains two identical tetramerized stacks per unit cell related by the $\mathrm{F}$ centering (and inversion) symmetry, the superstructure of (TMTTF) $)_{2} \mathrm{FSO}_{3}$ contains two different stacks which breaks the $\mathrm{F}$ symmetry [155]. The ordered $\mathrm{FSO}_{3}$ anions located between the stacks form ferroelectric chains. This removes also the inversion symmetry which relates the two donor stacks in (TMTTF) ${ }_{2} \mathrm{ReO}_{4}$ and lowers the space group symmetry to $\mathrm{P} 1$.

$(\mathrm{TMTSF})_{2} \mathrm{FSO}_{3}$ exhibits also at $87.5 \mathrm{~K}$ and atmospheric pressure a strongly 1 st order MI transition which stabilizes the $(1 / 2,1 / 2,1 / 2)$ AO superstructure [157], which, because of the ferroelectric order of the $\mathrm{FSO}_{3}$ [158], is probably non centro-symmetric as the TMTTF analogue. Under pressure the MI is removed and above $\sim 0.5 \mathrm{GPa}$ (TMTSF) ${ }_{2} \mathrm{FSO}_{3}$ becomes a superconductor with a $T_{\mathrm{S}}$ as high as $3 \mathrm{~K}[159,160]$. Preliminary X-ray diffraction measurements [158] show that in the pressure range of observation of superconductivity the $(1 / 2,1 / 2,1 / 2)$ superstructure is transformed into the $(0,0,1 / 2)$ superstructure, which itself transforms into the $(0,1 / 2,0)$ superstructure at higher pressure. Recent transport measurements provide evidence that the $(1 / 2,1 / 2,1 / 2)$ AO transition splits into several phases under pressure [160]. NMR studies [161] suggest that this splitting reveals the succession upon cooling of the ordering of the $\mathrm{FSO}_{3}$ without ordering of the F-sites then of the ordering of the F-sites (i.e. ordering of electric dipoles).

\subsection{The $(0,1 / 2,0)$ AO Transition of $(\text { TMTSF })_{2} \mathrm{ClO}_{4}$}

\subsubsection{Basic Aspects of the AO Superstructure and of Its Electronic Structure}

The AO transition of the (TMTSF) ${ }_{2} \mathrm{ClO}_{4}$ is significantly different from the AO transition of other salts of tetrahedral anions. For example in (TMTTF) ${ }_{2} \mathrm{ClO}_{4}$, the $\mathrm{ClO}_{4}$ adopts a staggered order in all directions without important interaction of the $\mathrm{ClO}_{4}$ with the organic stacks (see Section 7.2.2). In (TMTSF) ${ }_{2} \mathrm{ClO}_{4}$, the $\mathrm{ClO}_{4}$ adopts a staggered order in the $\mathrm{b}$ direction and a uniform order in a and $\mathrm{c}$ directions [61]. Surprisingly, the ordering of the $\mathrm{ClO}_{4}$ is accompanied by its shift which increases the closest Se-O distance [115] and a de-centering of the $\mathrm{ClO}_{4}$ inside its methyl group cavity [141]. This de-centering reinforces $\mathrm{H}$-bonds of the $\mathrm{ClO}_{4}$ with the methyl groups of the closest TMTSF (TMTSF A) 
and form loose contacts with the methyl groups of the other TMTSF (TMTSF B) of the cavity. The uniform shift of anions along a, thus stabilizes two types A and B of TMTSF stacks per unit cell.

The polarization of the methyl group cavities by the anion shift causes a charge transfer from stack A to stack B via Process 1 shown in Figure 9. TMTSF A towards which the anion moves becomes hole rich while TMTSF $B$ which has looser contacts becomes hole poor [21]. The charge transfer from A to B molecules drives an incommensurate band filling [147]. EHT [141] and more recent DFT [162] band structure calculations estimate this charge transfer at $\sim 0.025$ electrons per TMTSF. In a 1D picture, this deviation from the quarter band filling should kill the $4 \mathrm{k}_{\mathrm{F}}=\mathrm{a}^{*}$ umklapp electron-electron scattering terms which tend to promote the Mott-Hubbard gap.

Another important consequence of the AO transition is that TMTSF A towards which the $\mathrm{ClO}_{4}$ move is deformed (chair-like conformation), probably because of the constraints exercised by the $\mathrm{H}$ bonds, while unconstrained TMTSF B remains planar [141]. These different molecular deformations achieve different HOMO energies for A and B molecules (separated by $\sim 0.1 \mathrm{eV}[141,162]$ ). Also the non equivalent stacks $\mathrm{A}$ and $\mathrm{B}$ become differently dimerized.

The band structure and the FS of the AO superstructure have been calculated using EHT [141] and DFT [162,163]. All these calculations give an open FS composed of four quasi-1D sheets. The sheets originate from the "bonding" and "anti-bonding" combinations of the dimeric electronic structure of A and B stacks. Both the A-B inter-stack transfer integrals and the difference of site energies (including the HOMO energy separation plus the staggered anion potential) contribute to the warping and splitting of the FS. DFT calculation [162,163] shows that the anion gap has no significant effect on the FS shape and that the main contribution at the FS splitting comes from the difference of HOMO energies between the A and B donors. However the warping of the FS differs with the method of calculation [141,163]. In spite of this problem the assumption frequently done in the literature of a high temperature band structure only split by the staggered anion potential $[144,145]$ is not valid to interpret the data obtained below $T_{\mathrm{AO}}$.

In addition, as revealed by the most recent DFT calculations [162] and in contradiction with the earlier EHT calculation [141], the "bonding" and "anti-bonding" combinations of the dimeric electronic structure of A and B stacks lead to differently warped quasi-1D sheets that do not nest "perfectly" on each other. This could explain why the SDW ground state is not stabilized in the AO superstructure. This means also that the placement of (TMTSF) ${ }_{2} \mathrm{ClO}_{4}$ in the generic phase diagram built on octahedral anions [52] is ambiguous because the vanishing of the SDW ground state in AO $\mathrm{ClO}_{4}$ salt is not due to the enhanced warping of the FS, as it occurs under pressure in salts with octahedral anions such as $\mathrm{PF}_{6}$, but to the reconstruction of the band structure. A proof is that in quenched $\mathrm{ClO}_{4}$ salt (see below), where the band structure is not reconstructed by the AO process, the SDW ground state is kept. Another ambiguity with the placement of relaxed (TMTSF) ${ }_{2} \mathrm{ClO}_{4}$ in the generic phase diagram is that superconductivity occurs for an electronic structure substantially different of the pressurized (TMTSF) ${ }_{2} \mathrm{PF}_{6}$ one.

To summarize these recent findings, $\mathrm{AO}$ opens space for superconductivity in (TMTSF) ${ }_{2} \mathrm{ClO}_{4}$ by killing the SDW with the reconstruction of the electronic structure. AO also kills the tendency to form a $4 \mathrm{k}_{\mathrm{F}}$ commensurate Mott-Hubbard gap by achieving an inter-stack electronic charge transfer. 


\subsubsection{Texture of the AO Phase}

By its FS reconstruction AO controls the low temperature electronic properties of $\mathrm{TMTSF}_{2} \mathrm{ClO}_{4}$. In particular the tuning between SDW and superconductivity which results from this electronic structure modification is revealed using the slow kinetics required to orient the anions. The slow kinetics is due to the fact that $\mathrm{AO}$ occurs at a temperature $T_{\mathrm{AO}}=24 \mathrm{~K}$ much lower than the barrier height $(\sim 240 \mathrm{~K})$ necessary to overcome to change the orientation of the $\mathrm{ClO}_{4}$. The achievement of the AO phase thus requires a very slow cooling rate. In that case relaxed (TMTSF) ${ }_{2} \mathrm{ClO}_{4}$ becomes a superconductor with a $T_{\mathrm{S}} \sim 1.2 \mathrm{~K}$ [1]. For faster cooling rates the $\mathrm{AO}$ is incomplete and (TMTSF) ${ }_{2} \mathrm{ClO}_{4}$ undergoes a SDW order, due to the FS nesting, at a $\mathrm{T}_{\mathrm{SDW}}$ increasing with the cooling rate and capped to $\sim 6.5 \mathrm{~K}$ for the most rapidly cooled (quenched) samples [2]. Although it is often stated that a very slow cooling rate achieves a perfect $\mathrm{AO}$ and that a very rapid cooling rate leaves the anions disordered, high resolution $\mathrm{X}$-ray measurements [164] show that this assertion is incorrect: there is no true long range AO even in the best relaxed samples and a local AO is always present even in the best quenched samples. Also the cooling rate modifies progressively the fraction of ordered anions and the texture of the AO pattern, as we shall see below.

It is interesting to remark that, when $T_{\mathrm{AO}}$ is low (let say below $40 \mathrm{~K}$ ), the cooling rate affects the achievement of the $\mathrm{AO}$ process for anions, such as $\mathrm{ClO}_{4}$ and $\mathrm{NO}_{3}$ [165], weakly coupled to the TMTSF, but has no real effect for the $\mathrm{BF}_{4}$ anion [149] more strongly linked to the donors (see Section 7.2.1). This means that the use of a phenomenological model of a static double well of potential, external to the anion, to describe the kinetics of ordering is oversimplified especially if the ordering process involves a sizeable interaction with the TMTSF stack able to modify substantially the shape of this potential in order to lower the potential height to overcome during the ordering process.

Let us now discuss more quantitatively the texture of the AO domains. First, superconductivity occurs in $\mathrm{ClO}_{4}$ ordered domains. Figure 12 shows that, in function of the cooling rate, the fraction of ordered $\mathrm{ClO}_{4}$ [164] is identical to the fraction of the superconducting volume determined by the measurement of Meissner effect [166].

Figure 12. Fraction of ordered $\mathrm{ClO}_{4}$ (black symbols defined in [164]) and fraction of superconducting volume (red crosses) in (TMTSF) ${ }_{2} \mathrm{ClO}_{4}$ in function of the cooling rate. The fraction of superconducting volume has been determined from the data of [166] assuming that the relaxed sample is completely superconducting.

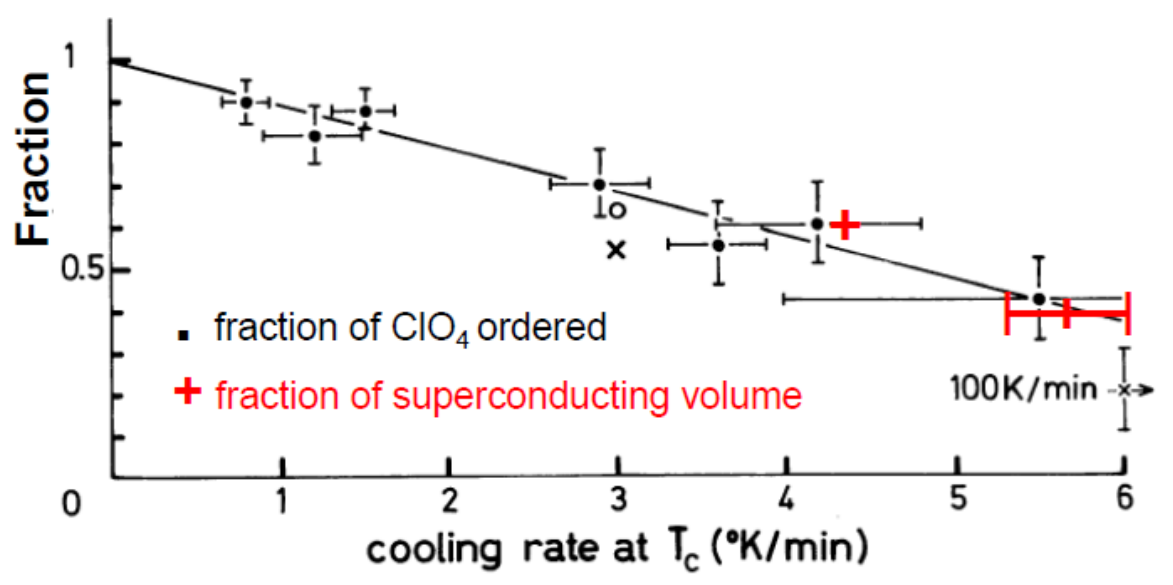


Second, the profile of the $(0,1 / 2,0)$ super-lattice reflections evolves with the cooling rate [164], which means that the topology of the AO domain pattern changes. The superlattice reflections of relaxed samples have a Lorentzian square profile (LS in Figure 13b) and those of quench samples, a Gaussian profile ( $\mathrm{G}$ in Figure 13a). The texture of relaxed samples (right part of Figure 13b) is made of a collection of domains where the $\mathrm{ClO}_{4}$ is ordered in one of its two opposite orientations (I and II in Figure 13b). Due to the intricate pattern of the domain walls and to the random distribution of domain walls there is along a given direction (straight line in Figure 13b) a large distribution of domain size, which Fourier transform gives a LS function [167]. The texture of quenched samples (right part of Figure 13a) is made of $\mathrm{AO}$ domains of about the same size and shape which are embedded in a matrix of disordered $\mathrm{ClO}_{4}$ (in grey in Figure 13a). With a small (i.e., Gaussian) distribution of domain sizes, the Fourier transform of the shape of ordered domains is a $\mathrm{G}$ function [168]. For intermediate cooling rates the profile of the $(0,1 / 2,0)$ superlattice reflections evolves from a LS to a $\mathrm{G}$ [164]. This means that non ordered $\mathrm{ClO}_{4}$ regions begin to develop between type I and II ordered domains.

Figure 13. Left part: profile of the same AO superstructure reflection of (TMTSF) $)_{2} \mathrm{ClO}_{4}$ in the (a) quenched and (b) relaxed states (data of [164]). Note the angular broadening (larger than the experimental resolution) of the superstructure reflection when the sample is either in the quenched or in the relaxed state. Right part: texture corresponding to the (a) quenched and (b) relaxed states. In (a) $\mathrm{L}$ is the typical size of a domain of ordered $\mathrm{ClO}_{4}, l$ is a typical inter-domain distance and the grey background represents non ordered $\mathrm{ClO}_{4}$. In (b) domains of types I and II with opposite $\mathrm{ClO}_{4}$ orientations are indicated. Along the line drawn in (b) there is a large distribution of domain sizes.
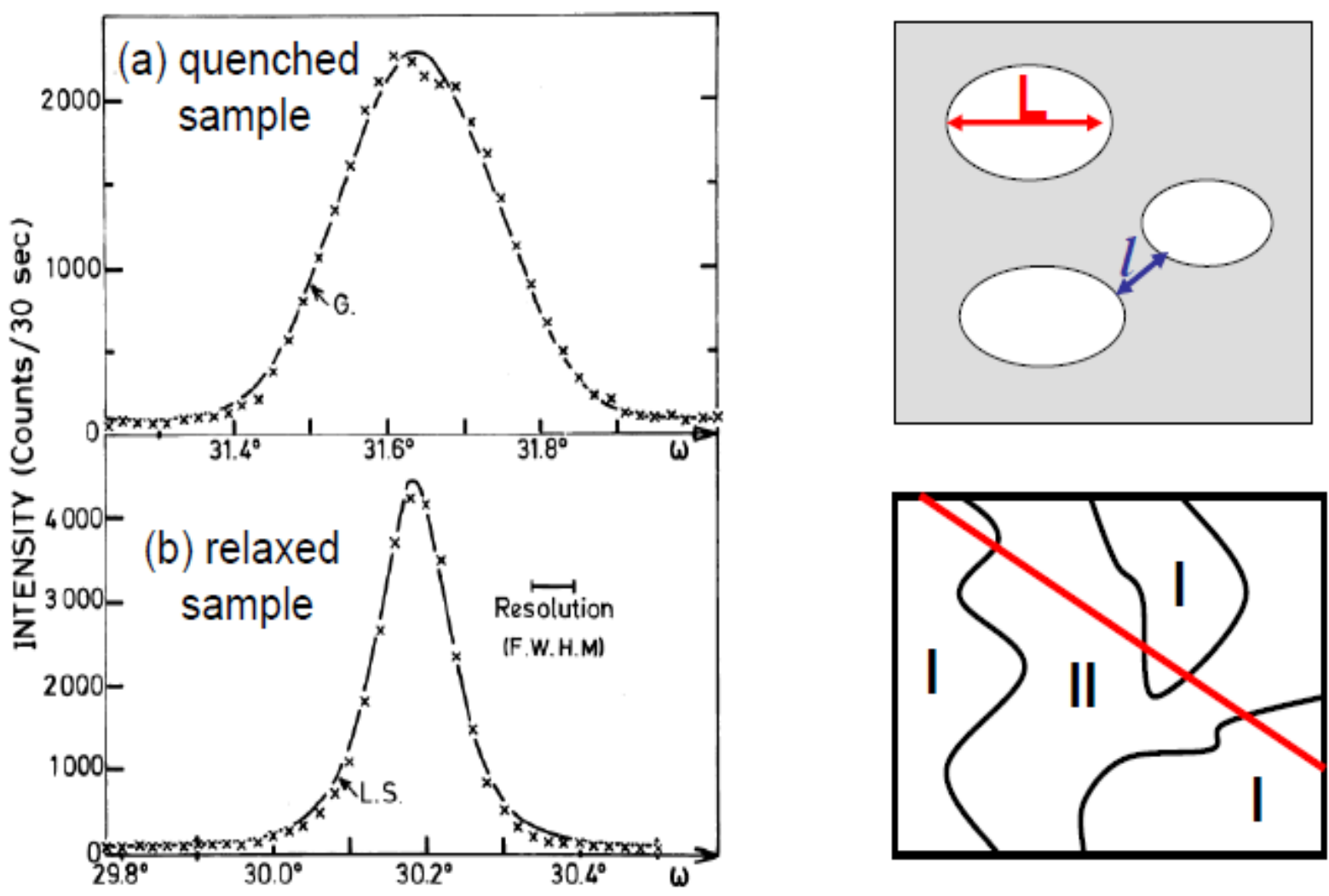
The change of topology, and the presence of additional regions of non ordered $\mathrm{ClO}_{4}$, has to be considered to interpret the cooling rate dependence of the superconductivity critical temperature, $T_{\mathrm{S}}$. For intermediate cooling rates, let say between $0.5 \mathrm{~K} / \mathrm{min}$ and $5 \mathrm{~K} / \mathrm{min}$, the reduction of the fraction of AO superconducting domains, given in Figure 12, is mostly due to the increase of the average distance, $l$, between the AO domains (by a factor 7) while average domain size L decreases more slowly (by a factor 2). For intermediate cooling rates $T_{\mathrm{S}}$, after a rapid drop, saturates. In presence of such a texture superconductivity is achieved through Josephson coupling between AO superconducting domains through the non ordered regions. The quantitative evolution of $T_{\mathrm{S}}$ has been calculated [169] using the dependence of $\mathrm{L}$ and $l$ with the cooling rate. It is found that the rate of decrease of $T_{\mathrm{S}}$ saturates in agreement with the experimental finding. Finally, for larger cooling rate, when L becomes smaller than the superconductivity coherence length, $\xi_{0}, T_{\mathrm{S}}$ rapidly drops to zero

In the solid solution (TMTSF) $)_{2}\left(\mathrm{ClO}_{4}\right)_{1-\mathrm{x}}\left(\mathrm{ReO}_{4}\right)_{\mathrm{x}}$, the profile of the superlattice reflections remains LS in the domain of existence of the $(0,1 / 2,0)$ local order [170]. However the probability $\mu$ to cross a domain wall per unit length [167] increases considerably with $x$. As $\mu^{-1}$ amounts to the average distance between $\mathrm{ReO}_{4}$ substituent for $x=5 \%$ and $7 \%$ [171], $\mathrm{ReO}_{4}$ appears to be very efficient to break the $(0,1 / 2,0) \mathrm{AO}$ spatial coherence. This can be easily understood since $\mathrm{ReO}_{4}$ develops different interactions with TMTSF (interaction 2 in Figure 9) than does $\mathrm{ClO}_{4}$ (interaction 1 in Figure 9) [172]. With this texture it is found, by the measurement $[173,174]$ of a quasi-linear decrease of $T \mathrm{~s}$ with $\mathrm{x}$ increasing, that $\mathrm{ReO}_{4}$ acts as non magnetic point defects on the superconductivity of (TMTSF) ${ }_{2} \mathrm{ClO}_{4}$. This behavior, interpreted as due to the reduction of the Cooper pair lifetime with the increasing number of defects, is taken as an evidence of the presence of nodes in the superconducting gap. However a microscopic theory linking this pair breaking effect at the local perturbations of the electronic structure due to the fragmentation of the $(0,1 / 2,0) \mathrm{AO}$ domain pattern with the $\mathrm{ReO}_{4}$ is lacking.

An important aspect of textured (TMTSF) ${ }_{2} \mathrm{ClO}_{4}$ is that the electronic parameters should vary spatially because the local order around the "ordered" and "non ordered" $\mathrm{ClO}_{4}$ is different. This is illustrated by the observation of a significant change of lattice parameters with the cooling rate. In particular the c parameter increases (decreases) in the quenched (relaxed) state with respect to the dependence extrapolated from the thermal variations above $T_{\mathrm{AO}}[175,176]$. Similarly the $\gamma$ angle increases (decreases) in the relaxed (quenched) state. The decrease of c, direction along which TMTSF and $\mathrm{ClO}_{4}$ layers alternate, has to be related to the contraction of the methyl group cavity when the ordered $\mathrm{ClO}_{4}$ establishes its $\mathrm{H}$-bonds in the relaxed state. The opening of $\gamma$ corresponds to a shear deformation of the TMTSF layer when molecule A adopts the boat shape. In addition, high resolution measurements [164] show that the growth of the AO order parameter upon cooling below $T_{\mathrm{AO}}$ is accompanied by erratic angular deformations of the lattice, consisting mostly in sudden rotations of the c direction probably due to the establishment of the H-bond network with newly ordered $\mathrm{ClO}_{4}$. Each angular jump, accompanied by a narrowing of the angular distribution of c directions, realizes an improvement of the crystalline order. Differently, the decrease of the AO order parameter upon heating is accompanied by the increase of the angular distribution of $\mathrm{c}$ directions due to the disorder induced by the successive breaks of the H-bonds. This shows that the AO process, via the progressive setting of the H-bond network, is accompanied by considerable elastic constraints. 


\subsection{The AO/CO Transitions of the SCN Salts}

The early discovery [94] of the 2nd order AO transition of (TMTTF) $)_{2} \mathrm{SCN}$ was a very important step to assess the importance of electron-electron repulsions in organic conductors. Indeed the stabilization of the $(0,1 / 2,1 / 2)$ superstructure below $T_{\mathrm{AO}}=160 \mathrm{~K}$ was the first realization of a MI transition [66] not accompanied by the opening of a gap in the spin degrees of freedom. In other words the $\mathrm{AO}$ of $(\mathrm{TMTTF})_{2} \mathrm{SCN}$ achieved the spin-charge decoupling expected from the stabilization of a $4 \mathrm{k}_{\mathrm{F}}$ periodicity. Recently the structural refinement of the AO structure [103] was able to prove from the variation of the intra-molecular distances that the AO transition is accompanied by a $4 k_{\mathrm{F}} \mathrm{CDW}$ of an amplitude of 0.15 electrons, with an excess of hole on the TMTTF towards which the SCN points and an excess of electron in the opposite situation. This transition is also accompanied by a reduction of the amplitude of the stack dimerization. This result is expected by the repulsive coupling between the $4 \mathrm{k}_{\mathrm{F}}$ CDW and $4 \mathrm{k}_{\mathrm{F}}$ BOW order parameters (this is exactly the opposite of the effect described in Section 6.2 and Annex A.3 where the establishment of the SP tetramerization reduced the amplitude of the CO). At low temperature $(\mathrm{TMTTF})_{2} \mathrm{SCN}$ undergoes at $T_{\mathrm{N}}=7 \mathrm{~K}$ [94] an AF transition which stabilizes the $\mathrm{q}_{\mathrm{AF}}=$ $(1 / 2,1 / 4$, ?) commensurate magnetic order [177].

(TMTSF) $)_{2} \mathrm{SCN}$ also undergoes a MI transition at $\sim 90 \mathrm{~K}$ which does not affect the spin degrees of freedom and which is also associated to a structural modulation [178]. Thus a " $4 \mathrm{k}_{\mathrm{F}}$ " charge localization should accompany this MI transition as in (TMTTF) ${ }_{2} \mathrm{SCN}$. However the structural modulation surprisingly corresponds to the establishment of a triply incommensurate short range order. The wave vector of this modulation $(0.48 \pm 0.015,0.65 \pm 0.01,0.1 \pm 0.02)$ is quite different to the one, $(0,1 / 2$, $1 / 2$ ), stabilized at the $\mathrm{CO} / \mathrm{AO}$ transition of $(\mathrm{TMTTF})_{2} \mathrm{SCN}$. It is however interesting to remark that the wave vector components of the incommensurate modulation of (TMTTF) ${ }_{2} \mathrm{SCN}$ approximately verify the relationship $\mathbf{q} \mathbf{d}_{1}=0$, i.e., $\mathrm{q}_{\mathrm{a}}-\mathrm{q}_{\mathrm{b}}+\mathrm{q}_{\mathrm{c}}=0$ (mod. 1) derived in [60], for the setting of CO via direct interactions (S...NCS) between anions and donors. As the SCN anion can only take two orientations, the incommensurability of the modulation is certainly related to the probability of occupancy of one of the two orientations of the anion. For such a wave the incommensurability observed in diffraction experiments results from a statistical average because locally the modulation must be commensurate. The perfection of such a wave is altered (i.e., the harmonic content of the modulation is reduced) if there is a broad distribution of commensurate domain sizes. In addition, if visualizing the modulation from the origin of the lattice there are increasing fluctuations on the position of successive domain walls, or equivalently cumulative fluctuations of domain size, the long range order is lost. The Fourier transform of such a modulation pattern leads to broadened satellite reflections. Similar features are observed for the incommensurate $\mathrm{CO}$ modulation of (DMtTTF $)_{2} \mathrm{ClO}_{4}[102]$.

\subsection{The $(1 / 2,0,0)$ AO Transition of the $\mathrm{NO}_{3}$ Salts}

Both (TMTSF) ${ }_{2} \mathrm{NO}_{3}$ and $(\mathrm{TMTTF})_{2} \mathrm{NO}_{3}$ undergo an $\mathrm{AO}$ transition at $T_{\mathrm{AO}}=41 \mathrm{~K}$ [143] and $50 \mathrm{~K}$ [157] respectively and which stabilizes the same $(1 / 2,0,0)$ superstructure. Even if this superstructure establishes the $2 \mathrm{k}_{\mathrm{F}}$ periodicity in stack direction, the $\mathrm{AO}$ transition has a weak influence on the electronic properties of the donor stack. 
The $(1 / 2,0,0)$ superstructure of $(\mathrm{TMTSF})_{2} \mathrm{NO}_{3}$ has been refined [165]. No substantial stack tetramerization and differentiation between the individual TMTSF are observed below $T_{\mathrm{AO}}$ (the doubling of stack periodicity should, in principle, differentiate two molecules per unit cell). This means that the $\mathrm{NO}_{3}$ anion orders without significantly perturbing its methyl group cavity. Such a result is expected because of the small size of the $\mathrm{NO}_{3}$ anion. As the organic array is not perturbed by the AO process the electronic structure remains basically unchanged below $T_{\mathrm{AO}}$ and (TMTSF) ${ }_{2} \mathrm{NO}_{3}$ does not become semi-metallic as previously predicted [144,145]. Its FS remains quasi-1D below $T_{\mathrm{AO}}$ [179] and available for a nesting instability. Indeed (TMTSF) ${ }_{2} \mathrm{NO}_{3}$ stabilizes a SDW ground state at $T_{\mathrm{SDW}}=9 \mathrm{~K}$ whose modulation wave vector, determined by NMR [180], is close to the wave vector of the SDW phase of (TMTSF) ${ }_{2} \mathrm{PF}_{6}[54,55]$.

(TMTTF $)_{2} \mathrm{NO}_{3}$ undergoes a $(1 / 2,0,0)$ AO transition below which the TMTTF stack is probably weakly tetramerized because a weak spin gap, less than $k_{\mathrm{B}} T_{\mathrm{AO}}[66,118]$, opens, resulting in the ground state of $(\mathrm{TMTTF})_{2} \mathrm{NO}_{3}$ being non-magnetic at the difference of the magnetic SDW ground state of $(\mathrm{TMTSF})_{2} \mathrm{NO}_{3}$.

\section{Conclusions}

We have reviewed the very rich panel of structural instabilities exhibited by the Bechgaard and Fabre salts. We have presented a coherent synthesis of the latest experimental developments and interpreted more quantitatively older results in a unified picture. This allows us to correlate the structural instabilities with the electronic, magnetic and superconducting properties of these salts.

The main conclusion is that structural degrees of freedom have a decisive influence on the physical properties the Bechgaard and Fabre salts; an influence which is underestimated in the present literature. Structural instabilities are not considered in the interpretation of the generic electronic phase diagram [52] giving the evolution of the crossovers and of the ground states exhibited by the octahedral anion salts in function of a generalized coordinate, which can be the size of the anion or the pressure although there is not a direct correspondence between these variables [17]. The current interpretation [51] describes the evolution of 1D instabilities and ground states by taking into account the variation of electronic interactions and couplings with pressure, nature of the donor and size of the anion. However we have shown in this review that the orientation disorder of the anions and of the methyl groups should influence the quasi-1D electronic structure in a manner which is not considered until now. The generic phase diagram accounts for the Mott-Hubbard charge localization, the crossover from a 1D Luttinger liquid to 2D and 3D Fermi liquids, the FS nesting instability leading to the density wave ground state and, with the deterioration of the nesting, the restoration of the metallic state which achieves superconductivity. However BOW, CO and ferroelectric intra-chain instabilities involving intra-stack electronic charge redistributions and inter-chain Coulomb couplings are not included in this description. In particular structural degrees of freedom which take into account the reaction of the lattice at electronic charge redistributions should directly control the development of charge instabilities and set the inter-chain coupling via a generalized electron-phonon coupling including the anion shift. Lattice degrees of freedom play also a role in SP, AF and SDW transitions via "magnetoelastic" couplings which are quite important in the Fabre and Bechgaard salts $[25,128,181])$. 
In this review we have also shown that anions set direct and/or indirect inter-chain coupling mechanisms allowing stabilizing CO, ferroelectric and SP ground states. Also the blockade of the anions in the methyl group cavities can be taken as responsible for the suppression of the CO and SP phase transitions under pressure and of the $2 \mathrm{k}_{\mathrm{F}} \mathrm{BOW}$ instability of (TMTSF) ${ }_{2} \mathrm{PF}_{6}$ at low temperature. In addition, the adiabaticity of the phonon field controlling the lattice dynamics in reaction at the charge redistribution must be considered. We provide here arguments showing that non-adiabaticity is relevant to explain the SP/AF phase diagram of Fabre salts.

Figure 14. $\mathrm{CO}$ (of amplitude $\pm \delta$ ) and $\mathrm{AF}$ exchange coupling $\mathrm{J} \mathbf{S}_{\mathbf{1}} \mathbf{S}_{\mathbf{2}}$ function of a generalized distortion parameter $\mathbf{u}$ of the organic stack. The drawing represents the extreme $\delta=0.5$ situation where the HOMO of the donor is alternatively occupied by one electron (ionic donor $\mathrm{D}^{+}$) and two electrons (neutral donor $\mathrm{D}^{0}$ ). In the drawing the pink dots represent the electrons. The holes, corresponding to the absence of one electron on $\mathrm{D}^{+}$, bear the spins $\mathbf{S}_{\mathbf{1}}$ and $\mathbf{S}_{\mathbf{2}}$. The exchange interaction $\mathbf{J}(\mathbf{u})$ is tuned by the position, $\mathbf{u}$, of the neutral molecule which controls the intra-stack overlap of the HOMO's. The figure shows also that the molecular shift $\mathbf{u}$ changes the orientation of the electric dipole $\mathbf{P}$ of the $\mathrm{D}^{0} \mathrm{D}^{+}$dimer.

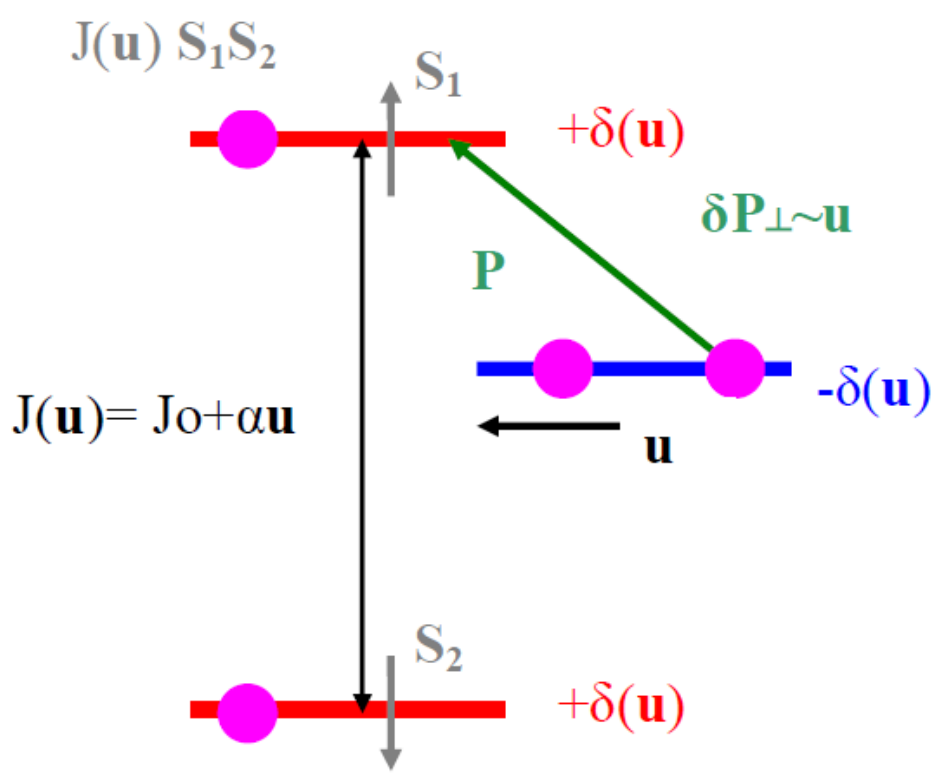

The generic phase diagram does not include salts with non centro-symmetrical anions such as those with $\mathrm{ReO}_{4}$ or $\mathrm{FSO}_{3}$ which become superconductors at larger $T_{\mathrm{S}}$ than $T_{\mathrm{S}}$ of salts with octahedral anions. In these salts, AO transitions, involving important charge redistributions, activate CDW and BOW instabilities of the organic stack. These charge displacements are accompanied by substantial deformations of the lattice structure. The modification of the electronic structure which thus results drives noticeable change of electronic properties. In this manner non centro-symmetrical anions can tune novel ground state competitions. Also little is known regarding the general pressure evolution of the $\mathrm{AO}$ ground states which, for salts with $\mathrm{ReO}_{4}$ and $\mathrm{FSO}_{3}$ anions stabilize new metallic superstructures which exhibit superconductivity, some of them being probably non-centrosymmetric when a ferroelectric dipolar anion order is achieved.

A beautiful illustration of the interplay between SDW and superconductivity is shown by (TMTSF) ${ }_{2} \mathrm{ClO}_{4}$. In this salt we have shown that this competition is controlled by the texture of the AO 
pattern. This is a simple case where superconductivity and SDW can be simply associated to structurally different regions. Texture effects are also important in the competition between SDW and superconductivity in pressurized (TMTSF) ${ }_{2} \mathrm{PF}_{6}$ [182]. In a general way it is interesting to remark that, similarly to pressurized (TMTSF) ${ }_{2} \mathrm{PF}_{6}$, the highest $T_{\mathrm{S}}$ in pressurized (TMTSF) ${ }_{2} \mathrm{ReO}_{4}[151,153]$ and $\mathrm{FSO}_{3}[159,160]$ is achieved in the de-mixing region between different AO phases.

Finally we conclude on the possible interplay between ferroelectricity and (antiferro-) magnetism in the Fabre salts which could give rise to a multiferroic behavior [183] where the action on the dielectric polarization reacts on the magnetic polarization and vice-versa. In particular, $\mathrm{CO}$ and the associated lattice deformation could control via a sizeable spin-phonon coupling the magnitude of the exchange interaction and the direction of the dielectric polarization as schematically illustrated by Figure 14. In addition, dipolar anions located between donor stacks could add a new degree of freedom at the ferroelectricity. Multiferroicity is now well documented in inorganic materials (see for example [184,185]). Its manifestation in spin-charge decoupled organic materials such as the ones discussed in this review deserve special attention in the future.

\section{Conflict of Interest}

The author declares no conflict of interest.

\section{Acknowledgments}

The work reported here is due to wide collaborations which have been quoted in the references. For the most recent achievements outlined in this review, useful discussions with E. Canadell, S. Charfi-Kaddour, C. Coulon, M. de Souza, M. Dressel, G. Giovannetti, S. Haddad, P. Foury-Leylekian, M. Lang, M. Poirier, E. Rose and H. Sawa are acknowledged. The author is also very grateful to C. Bourbonnais for a general discussion on the content of the paper.

\section{Appendix: Landau Theory with Coupled Order Parameters Applied at the Phase Diagram of the Bechgaard and Fabre Salts}

\section{A.1. The AF Phase of $(T M T T F)_{2} B r$}

The primary order parameter $\eta(\mathrm{q})$ describes the AF modulation. This two components AF order parameter, where $\mathrm{q}_{\mathrm{AF}}=(1 / 2, \pm 1 / 4, ?)$, can be represented by the complex number $\eta \mathrm{e}^{\mathrm{i} \theta}$. To the structural modulation, with $\mathrm{q}_{\mathrm{s}}=(1,1 / 2, ?)$, corresponds the real order parameter $\rho$. The Landau development of the free energy, which is function of $\eta, \theta$ and $\rho$, contains 3 contributions corresponding respectively in expression (A1) to (a) AF coupling, (b) cost of elastic deformation and (c) magneto-elastic coupling:

$$
F(\eta, \theta, \rho)=F_{A F}(\eta, \theta)+F_{E}(\rho)+F_{M E}(\eta, \theta, \rho) \text {, where: }
$$$$
\text { (a) } \mathrm{F}_{\mathrm{AF}}(\eta, \theta)=\mathrm{a} \eta^{2}+\mathrm{b} \eta^{4}+\mathrm{u} \eta^{4} \cos 4 \theta
$$$$
\text { (b) } F_{E}(\rho)=e \rho^{2}
$$

(c) $\mathrm{F}_{\mathrm{ME}}(\eta, \theta, \rho)=\lambda \rho \eta^{2} \cos 2 \theta$ 
The 3rd term in $\mathrm{F}_{\mathrm{AF}}(\eta, \theta)$ is the umklapp contribution due to commensurability 4 of the modulation: $4 \mathrm{q}_{\mathrm{AF}}$ is a reciprocal lattice wave vector. $\theta$ fixes the phase of the AF modulation with respect to the lattice. The minimization of $\mathrm{F}(\eta, \theta, \rho)$ with respect to $\rho$ gives :

$$
\begin{gathered}
\rho=(-\lambda / 2 \mathrm{e}) \eta^{2} \cos 2 \theta, \text { leading to: } \\
\mathrm{F}_{\mathrm{E}}(\rho)+\mathrm{F}_{\mathrm{ME}}(\eta, \theta, \rho)=\left(-\lambda^{2} / 8 \mathrm{e}\right) \eta^{4}(1+\cos 4 \theta)
\end{gathered}
$$

In (A2), $\rho$ is proportional to the square of the magnetization and depends on the phase $\theta$ of the AF modulation. $\left(-\lambda^{2} / 8 \mathrm{~d}\right)$ renormalizes the $4 \mathrm{rd}$ order coefficients $\mathrm{b}$ and $\mathrm{u}$, which thus become $b^{\prime}=b-\left(\lambda^{2} / 8 \mathrm{e}\right)$ and $u^{\prime}=u-\left(\lambda^{2} / 8 \mathrm{e}\right)$. By replacing $\rho$ by expression (A2) in (A1), $F(\eta, \theta)$ reads:

$$
\mathrm{F}(\eta, \theta)=\mathrm{a} \eta^{2}+\mathrm{b}^{\prime} \eta^{4}+\mathrm{u}^{\prime} \eta^{4} \cos 4 \theta
$$

As the AF transition of (TMTTF $)_{2} \mathrm{Br}$ is of 2 nd order one has $b^{\prime}>0$ in (A3). (A3) is minimum with respect to $\theta$ for $\sin 4 \theta=0$. This leads to $\theta=p \pi / 4$, with $p$ integer, from which two types of free energy $F(\eta)$ can be obtained:

$$
\begin{aligned}
& \text { (a): } \theta=0 \text { or } \pi / 2 \text {, leading to } F(\eta)=a \eta^{2}+\left[b+u-\left(\lambda^{2} / 4 e\right)\right] \eta^{4} \\
& \text { (b): } \theta=\pi / 4 \text { or } 3 \pi / 4 \text {, leading to } F(\eta)=a \eta^{2}+(b-u) \eta^{4}
\end{aligned}
$$

The minimization of $F(\eta)$ with respect to $\eta$ fixes the primary order parameter which is non-zero when $\mathrm{a}<0\left(\mathrm{a}=0\right.$ at $\left.\mathrm{T}_{\mathrm{N}}\right)$. $\rho$ is related to $\eta$ and $\theta$ by the expression (A.2). The two classes of solutions of (A4) are schematically represented in Figure 6 . The magneto-elastic coupling $\lambda$ favours solution (a) while the umklapp term $u$ favours solution (b). Solution (a) is the most stable if $\lambda^{2}>8$ eu. The structural order parameter $\rho$ is different from zero for solution (a) since the magneto-elastic coupling provides an energy gain only if $\eta(q)$ located on one site out of two. The charge modulation which thus results corresponds to a $4 \mathrm{k}_{\mathrm{F}} \mathrm{CDW}$ or a $\mathrm{CO}$.

This simple model ignores the stack dimerization otherwise a magnetoelastic BOW deformation could be achieved in solution (b). In this case there is an energy gain to put the parallel magnetizations on the intra-dimer bond and to shorten this bond. This shortening will favour the delocalization of the hole bearing the spin between the two sites forming the dimer.

\section{A.2. The Density Wave Phases of (TMTSF $)_{2} P F_{6}$}

According to data reported in Section 4.2, the analysis of the density wave phase transition should include 3 order parameters related to the $2 \mathrm{k}_{\mathrm{F}} \operatorname{SDW}\left(\eta_{1}(\mathrm{q})\right), 2 \mathrm{k}_{\mathrm{F}} \operatorname{CDW}\left(\eta_{2}(\mathrm{q})\right)$, and $4 \mathrm{k}_{\mathrm{F}} \operatorname{CDW}(\rho)$ modulations. As in Annex A.1, the first two order parameters are complex quantities and the third one is real. As qSDW is incommensurate there is no umklapp term in the free energy. In addition because of the incommensurability only the phase difference $\omega$ between the two complex order parameters $\eta_{1}$ and $\eta_{2}$ is relevant. There is also a phase shift $\theta$, already considered in the Annex A.1, between $\eta_{2}$ and $\rho$. In order to perform a derivation as simple as possible we shall ignore $\rho$ and the phase shift $\theta$ (in Annex A.1 expression (A.4) shows that the minimization of the free energy with respect to $\rho$ and $\theta$ leads to a renormalization of the $4^{\text {rd }}$ order coefficient of the primary order parameter). The Landau development of the free energy $F\left(\eta_{1}, \eta_{2}, \omega\right)$ is given by:

$$
F\left(\eta_{1}, \eta_{2}, \omega\right)=F\left(\eta_{1}\right)+F\left(\eta_{2}\right)+F_{C}\left(\eta_{1}, \eta_{2}, \omega\right), \text { where: }
$$




$$
\begin{gathered}
F\left(\eta_{i}\right)=a_{i} \eta_{i}{ }^{2}+b_{i} \eta_{i}^{4} \\
F_{C}\left(\eta_{1}, \eta_{2}, \omega\right)=\mu \eta_{1}{ }^{2} \eta_{2}^{2}+v \eta_{1}{ }^{2} \eta_{2}{ }^{2} \cos 2 \omega
\end{gathered}
$$

The free energy is minimum if $\omega=0(=\pi / 2)$ for negative (positive) $v$. This leads to a coupling term:

$$
\mathrm{F}_{\mathrm{C}}\left(\eta_{1}, \eta_{2}\right)=\mathrm{c} \eta_{1}^{2} \eta_{2}^{2} \text {, where } \mathrm{c}=\mu-|v|
$$

Figure A1. Phase diagrams of the Landau free energy, written on top of the figure, in function of the temperature $(\mathrm{T})$ and of an external parameter, $h$, allowing the variation of the Landau coefficients $a_{i}$. In the figure both $b_{1}$ and $b_{2}$ are positive. The value and sign of the coupling $\mathrm{c}$ between the order parameters are indicated for each phase diagram. Thin (thick) lines represent $2^{\text {nd }}\left(1^{\text {st }}\right)$ order transition lines. The phases are labeled by the order parameter which differs from zero. " 0 " corresponds to the non modulated $\left(\eta_{1}=\eta_{2}=0\right)$ phase. The critical temperature achieving pure $\eta_{1}$ or $\eta_{2}$ phases on each side of the phase " 0 " trough $2^{\text {nd }}$ order transitions (thin green lines) is given by $\mathrm{a}_{1}=0$ or $\mathrm{a}_{2}=0$ respectively. The arrows connected to the $2^{\text {nd }}$ order transition lines (thin red (blue) lines) delimiting the $\left(\eta_{1}, \eta_{2}\right)$ phase for $0<c<2 \sqrt{ }\left(b_{1} b_{2}\right)\left(0>c>-2 \sqrt{ }\left(b_{1} b_{2}\right)\right)$ indicate the sense of displacement of the phase boundaries when $c$ increases (c decreases). This displacement shows, when $c>2 \sqrt{ }\left(b_{1} b_{2}\right)$, that the $\left(\eta_{1}, \eta_{2}\right)$ phase collapses to a first order transition (thick red line) linking the pure $\eta_{1}$ and $\eta_{2}$ phases. When $c<-2 \sqrt{ }\left(b_{1} b_{2}\right)$, the expansion of the $\left(\eta_{1}, \eta_{2}\right)$ phase renders this phase directly reachable from the " 0 " phase through a first order phase transition (thick blue line).

\section{$\mathrm{F}=\mathrm{F}_{0}+\mathrm{a}_{1}(\mathrm{~T}, \mathrm{~h}) \mathrm{\eta}_{1}{ }^{2}+\mathrm{a}_{2}(\mathrm{~T}, \mathrm{~h}) \mathrm{\eta}_{2}{ }^{2}+\mathrm{b}_{1} \boldsymbol{\eta}_{1}{ }^{4}+\mathrm{b}_{2} \mathrm{\eta}_{2}{ }^{4+}$ $\mathbf{c} \eta_{1}^{2} \eta_{2}^{2}+f_{6}\left(\eta_{1}, \eta_{2}\right)$}
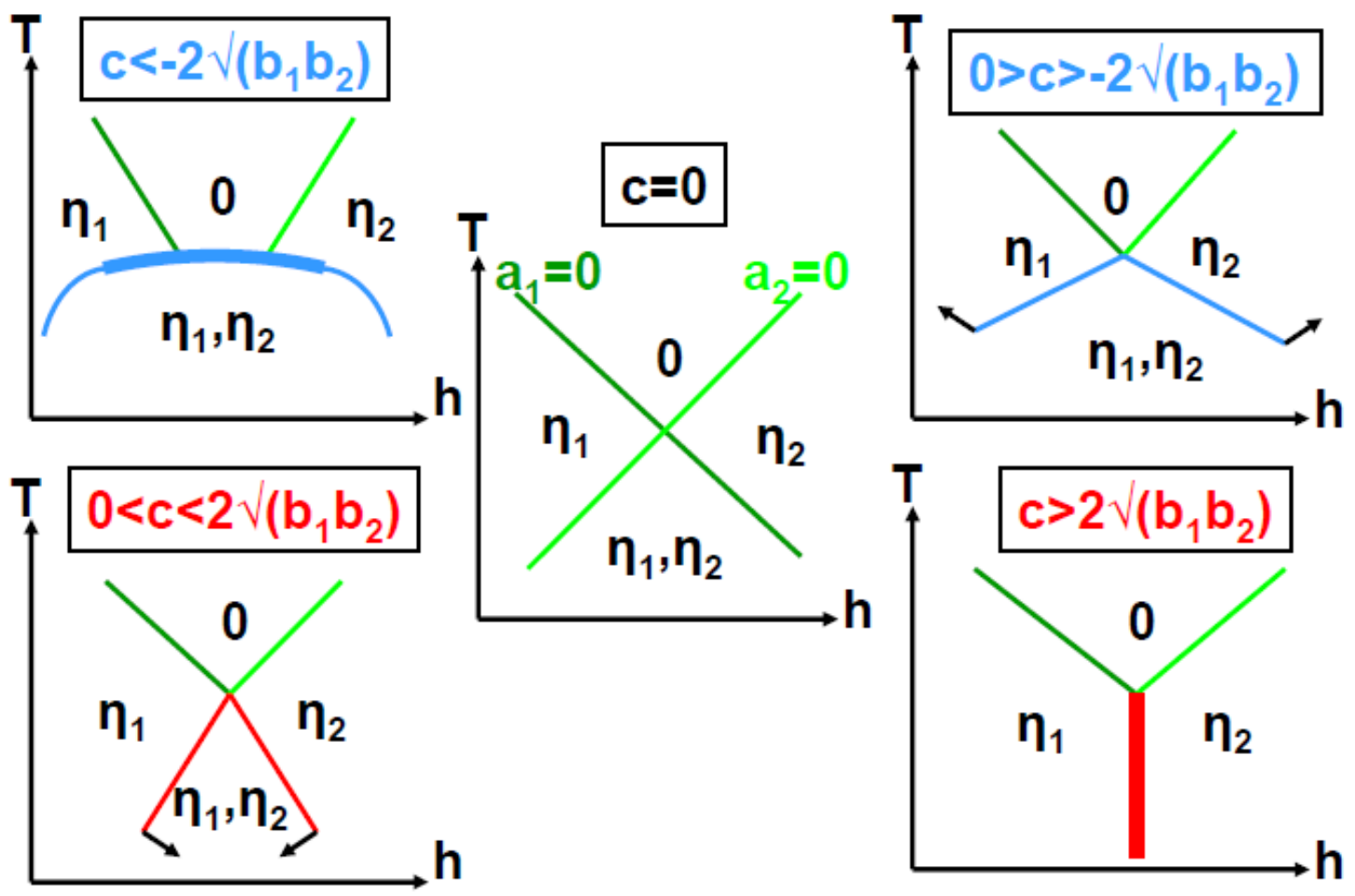
The functional (A5) with $\mathrm{F}_{\mathrm{C}}$ given by (A6), together with the presence of additional $6^{\text {th }}$ order terms providing the stability of the phases, has been studied in the literature $[186,187,85]$. These works lead to elaborated phase diagrams depending upon the value and sign of the coefficients entering in the Landau development. The results for positive $b_{1}$ and $b_{2}$ are summarized in Figure A1. Figure A1 shows that for an attractive enough coupling, $c<-2 \sqrt{ }\left(b_{1} b_{2}\right)$, between the $2 k_{F}$ SDW and $2 k_{F}$ CDW order parameters the SDW/CDW modulated phase can be reached trough a first order transition even if $b_{1}$ and $b_{2}$ coefficients of the 4 th order terms are positive (see also Figure $7 \mathrm{a}$ ). This peculiar feature was first noticed in [186]. This result requires going beyond the standard theory reported in [187], which, being restricted to $c>-2 \sqrt{ }\left(b_{1} b_{2}\right)$, gives a transition to the mixed phase only through a peculiar point corresponding to the simultaneous vanishing: $\mathrm{a}_{1}=\mathrm{a}_{2}=0$. A more general treatment considering negative $b_{i}$ coefficients has been developed in [85]. This theory leads to phase diagrams stabilizing the mixed $\left(\eta_{1}, \eta_{2}\right)$ phases if one or two $b_{i}$ coefficients are negative. All these phase diagrams are summarized in Figure 7. The stabilization of the $\left(\eta_{1}, \eta_{2}\right)$ phase implies that $c$ should be negative (attractive interaction between the $2 k_{\mathrm{F}} \mathrm{SDW}$ and the $2 k_{\mathrm{F}} \mathrm{CDW}$ ) and that $\mathrm{b}_{1}$ and $\mathrm{b}_{2}$ are small or even negative. c negative means $0<\mu<|v|$ or $\mu<0$. The condition $b_{1}$ and $b_{2}$ small or even negative is obtained if there is an efficient renormalization of the $b_{i}$ 's due to a strong coupling between the $2 \mathrm{k}_{\mathrm{F}}$ SDW and/or the $2 \mathrm{k}_{\mathrm{F}} \mathrm{CDW}$ and the secondary order parameter $\rho$ corresponding to the $4 \mathrm{k}_{\mathrm{F}} \mathrm{CDW}$. This renormalization is due to the magneto-elastic coupling previously considered in Annex A.1.

\section{A.3. Competition between $C O$ and SP Transitions}

We assume that CO develops first and that the SP transition occurs inside the CO phase. These two modulated states are characterized by the real order parameters $\eta_{\mathrm{CO}}$ and $\eta_{\mathrm{SP}}$, characterized by the $\mathrm{q}_{\mathrm{CO}}=(1,0,0)$ and $\mathrm{q}_{\mathrm{SP}}=(1 / 2,1 / 2,1 / 2)$ wave vectors respectively. When $\eta_{\mathrm{CO}}$ is already established, the Landau development of the free energy for the SP order parameter is given by:

$$
\begin{gathered}
\mathrm{F}\left(\eta_{\mathrm{CO}}, \eta_{\mathrm{SP}}\right)=\mathrm{F}\left(\eta_{\mathrm{CO}}\right)+\mathrm{F}\left(\eta_{\mathrm{SP}}\right)+\mathrm{F}_{\mathrm{C}}\left(\eta_{\mathrm{CO}}, \eta_{\mathrm{SO}}\right), \text { where: } \\
\mathrm{F}\left(\eta_{\mathrm{SP}}\right)=\mathrm{a}_{\mathrm{SP}} \eta_{\mathrm{SP}}^{2}+\mathrm{b}_{\mathrm{SP}} \eta_{\mathrm{SP}}^{4} \\
\mathrm{~F}_{\mathrm{C}}\left(\eta_{\mathrm{CO}}, \eta_{\mathrm{SP}}\right)=\lambda \eta_{\mathrm{CO}} \eta_{\mathrm{SP}}^{2}+\mu \eta_{\mathrm{CO}}{ }^{2} \eta_{\mathrm{SP}}^{2}
\end{gathered}
$$

Note that the Landau development in $\eta_{\mathrm{SP}}$ implies the presence of an adiabatic phonon field. In (A7) one has $a_{\mathrm{SP}}=\alpha_{\mathrm{SP}}\left(T-T_{\mathrm{SP}}{ }^{0}\right), b_{\mathrm{SP}}>0$. We shall assume in the following that $\eta_{\mathrm{CO}}(T)$ is enough small so that its value near $T_{\mathrm{SP}}$ can still be obtained by the minimization of the two first terms in the development of the $\mathrm{CO}$ free energy given by:

$$
\mathrm{F}\left(\eta_{\mathrm{CO}}\right)=\mathrm{a}_{\mathrm{CO}} \eta_{\mathrm{CO}}^{2}+\mathrm{b}_{\mathrm{CO}} \eta_{\mathrm{CO}}^{4}
$$

Then the minimization $\partial \mathrm{F}\left(\eta_{\mathrm{CO}}\right) / \partial \eta_{\mathrm{CO}}=0$ leads to:

$$
\eta_{\mathrm{CO}}(T)=\left[\alpha_{\mathrm{CO}}\left(T-T_{\mathrm{CO}}\right) / 2 \mathrm{~b}_{\mathrm{CO}}\right]^{1 / 2}
$$

In the following we shall assume that $\eta_{\mathrm{CO}}$ remains small enough so that only the lowest order $\lambda$ coupling term will be considered in $\mathrm{F}_{\mathrm{C}}\left(\eta_{\mathrm{CO}}, \eta_{\mathrm{SP}}\right)$. With these approximations $\eta_{\mathrm{CO}}$ renormalizes the Landau coefficient asp, which thus becomes: 


$$
\mathrm{a}_{\mathrm{SP}}^{\prime}=\mathrm{a}_{\mathrm{SP}}+\lambda \eta_{\mathrm{CO}}=\alpha_{\mathrm{SP}}\left(T-T_{\mathrm{SP}}\right)
$$

This leads to a renormalization of the critical temperature $T_{\mathrm{SP}}{ }^{0}$, given by $\mathrm{a}_{\mathrm{SP}}=\alpha_{\mathrm{SP}}\left(T-T_{\mathrm{SP}}{ }^{0}\right)$, to $T_{\mathrm{SP}}$ defined by (A.10). With $T_{\mathrm{SP}}$ much smaller than $T_{\mathrm{CO}}$ one gets:

$$
T_{\mathrm{SP}}=T_{\mathrm{SP}}^{0}-\lambda \eta_{\mathrm{CO}}\left(T_{\mathrm{SP}}\right) / \alpha_{\mathrm{SP}}
$$

With $\eta_{\mathrm{CO}}\left(T_{\mathrm{SP}}\right)$ given by (A9), (A11) becomes:

$$
T_{\mathrm{SP}}=T_{\mathrm{SP}}{ }^{0}-\mathrm{A}\left(T_{\mathrm{CO}}-T_{\mathrm{SP}}\right)^{1 / 2}, \text { with } \mathrm{A}=\left(\lambda / \alpha_{\mathrm{SP}}\right)\left[\alpha_{\mathrm{CO}} / 2 \mathrm{~b}_{\mathrm{CO}}\right]^{1 / 2}
$$

The relationship (A12) between $T_{\mathrm{SP}}$ and $T_{\mathrm{CO}}$ is drawn in Figure 11. Note that if the $\mu$ biquadratic term of $F_{C}\left(\eta_{\mathrm{CO}}, \eta_{\mathrm{SP}}\right)$ is considered instead of the $\lambda$ linear-quadratic term there is a linear relationship between $T_{\mathrm{SP}}$ and $T_{\mathrm{CO}}$. This linear plot is shown in Figure 12 of [73].

The minimization of $(\mathrm{A} 7)+(\mathrm{A} 8)$ with respect to $\eta_{\mathrm{CO}}$ leads to a decrease of $\eta_{\mathrm{CO}}(T)$ below $T_{\mathrm{SP}}$, when the repulsive coupling $\mathrm{F}_{\mathrm{C}}\left(\eta_{\mathrm{CO}}, \eta_{\mathrm{SP}}\right)$ develops with the growth of $\eta_{\mathrm{SP}}$. The (negative) relative correction $\Delta \eta_{\mathrm{CO}}(T) / \eta_{\mathrm{CO}}\left(T_{\mathrm{SP}}\right)$ at the $\eta_{\mathrm{CO}}$ order parameter variation given by (A9) is, in first order in $\lambda$ :

$$
\begin{gathered}
\Delta \eta_{\mathrm{CO}}(T) / \eta_{\mathrm{CO}}\left(T_{\mathrm{SP}}\right) \approx-\left[\lambda \eta_{\mathrm{SP}}(T)^{2}\right] /\left[8 \mathrm{~b}_{\mathrm{CO}} \eta_{\mathrm{CO}}\left(T_{\mathrm{SP}}\right)^{3}\right], \text { where: } \\
\eta_{\mathrm{SP}}(T)^{2}=\alpha_{\mathrm{SP}}\left(T-T_{\mathrm{SP}}\right) / 2 \mathrm{~b}_{\mathrm{SP}}
\end{gathered}
$$

The $\mu$ biquadratic term in $\mathrm{F}_{\mathrm{C}}\left(\eta_{\mathrm{CO}}, \eta_{\mathrm{SP}}\right)$ leads also to a negative correction proportional to $\eta_{\mathrm{SP}}(T)^{2}$.

\section{References and Notes}

1. Jérome, D.; Schulz, H.J. Organic conductors and superconductors. Adv. Phys. 1982, 31, 299-490.

2. Ishiguro, T.; Yamaji, K.; Saito, G. Organic Super-Conductors, 2nd ed.; Springer-Verlag: Berlin/Heidelberg, Germany, 1998; Volume 88.

3. Toyota, N.; Lang, M.; Müller, J. Low-Dimensional Molecular Metals; Springer-Verlag: Berlin/Heidelberg, Germany, 2007; Volume 154.

4. Henriques, R.T.; Alcácer, L.; Pouget, J.P.; Jérome, D. Electrical conductivity and x-ray diffuse scattering study of organic conductors (perylene $)_{2} \mathrm{M}(\mathrm{mnt})_{2},(\mathrm{M}=\mathrm{Pt}, \mathrm{Pd}, \mathrm{Au})$. J. Phys. C: Solid State Phys. 1984, 17, 5197-5208.

5. Rovira, C.; Veciana, J.; Ribera, E.; Tarrès, J.; Canadell, E.; Rousseau, R.; Mas, M.; Molins, E.; Almeida, M.; Henriques, R.T.; et al. An organic spin-ladder molecular material. Angew. Chem. Int. Ed. 1997, 36, 2324-2326.

6. Canadell, E.; Rachidi, I. E.-I.; Ravy, S.; Pouget, J.-P.; Brossard, L.; Legros, J.P. On the band electronic structure of $\mathrm{X}\left[\mathrm{M}(\mathrm{dmit})_{2}\right]_{2}\left(\mathrm{X}=\mathrm{TTF},\left(\mathrm{CH}_{3}\right)_{4} \mathrm{~N} ; \mathrm{M}=\mathrm{Ni}, \mathrm{Pd}\right)$ molecular conductors and superconductors. J. Phys. France 1989, 50, 2967-2981.

7. Pouget, J.-P.; Ravy, S. Structural aspects of the bechgaard salts and related compounds. J. Phys. I France 1996, 6, 1501-1525.

8. Pouget, J.-P. Structural features of the Bechgaard salts and related compounds. J. Phys. IV France 2000, 10, Pr3-43-56. 
9. Thorup, N.; Rindorf, G.; Soling, H.; Bechgaard, K. The structure of Di(2,3,6,7-tetramethyl1,4,5,8-tetraselenafulvalenium) Hexafluorophosphate, (TMTSF) ${ }_{2} \mathrm{PF}_{6}$, the First Superconducting Organic Solid. Acta Cryst. B 1981, 37, 1236-1240.

10. Pashkin, A.; Dressel, M.; Ebbinghaus, S.G.; Hanfland, M.; Kuntscher, C.A. Pressure-induced structural phase transition in the Bechgaard-Fabre salts. Synth. Met. 2009, 159, 2097-2100.

11. Gallois, B.; Gaultier, J.; Hauw, C.; Lamcharfi, T.; Filhol, A. Neutron Low-Temperature (4 and $20 \mathrm{~K})$ and X-ray High-Pressure $\left(6.5 \times 10^{2}\right.$ and $\left.9.8 \times 10^{2} \mathrm{MPa}\right)$ Structures of the Organic Superconductor Di(2,3,6,7-tetramethyl-1,4,5,8-tetraselenafulvalenium) Hexafluorophosphate, (TMTSF) ${ }_{2} \mathrm{PF}_{6}$. Acta Cryst. B 1986, 42, 564-575.

12. Emery, V.J.; Bruinsma, R.; Barisic, S. Electron-electron umklapp scattering in organic superconductors. Phys. Rev. Lett. 1982, 48, 1039-1043.

13. Ishibashi, S.; Manuel, A.A.; Kohyama, M. Ab initio pseudopotential calculation for (TMTSF) ${ }_{2} \mathrm{ClO}_{4}$. J. Phys. Cond. Matt. 1999, 11, 2279-2283.

14. Liautard, B.; Peytavin, S.; Brun, G.; Maurin, M. Corrélations structurales dans la série (TMTTF) $)_{2}$ X. J. Phys. (Paris) 1982, 43, 1453-1459.

15. Liautard, B.; Peytavin, S.; Brun, G.; Maurin, M. Structural studies and physical properties in the organic conductors series (TMTTF) ${ }_{2} \mathrm{X}$ and (TMTSF) $)_{2}$ X. J. Phys. (Paris) 1983, 44, C3-951-956.

16. Kistenmacher, T.J. Cavity size versus anion size in (TMTSF $)_{2} \mathrm{X}$ salts: Possible implications for the uniqueness of (TMTSF) ${ }_{2} \mathrm{ClO}_{4}$. Solid State Commun. 1984, 50, 729-733.

17. This assertion is incorrect for TMTSF and TMTSF salts because the anion modifies mainly the $c$ parameter $[14,15]$ while pressure leads to a relative decrease of the parameter a twice larger than for parameters $b$ and $c[10,11]$.

18. Kistenmacher, T.J. Anion-donor coupling in (TMTSF) $)_{2} \mathrm{X}$ salts: Symmetry considerations. Solid State Commun. 1984, 51, 931-934.

19. Beno, M.A.; Blackman, G.S.; Leung, P.C.W.; Williams, J.M. Hydrogen bond formation and anion ordering in superconducting (TMTSF) ${ }_{2} \mathrm{ClO}_{4}$ and $(\mathrm{TMTSF})_{2} \mathrm{AsF}_{6}$. Solid State Commun. 1983, 48, 99-103.

20. Granier, T.; Gallois, B.; Fritsch, A.; Ducasse, L.; Coulon, C. 135K Crystallographic and Electronic Structure of $(\mathrm{TMTTF})_{2} \mathrm{SbF}_{6}$. In Lower-Dimensional Systems and Molecular Electronics; Metzger, R.M., Day, P., Papavassiliou, G.C., Eds; Plenum Press: New York, NY, USA, 1990; Volume 248, pp. 163-168.

21. Pouget, J.-P.; Foury-Leylekian, P.; Alemany, P.; Canadell, E. Charge ordering in low dimensional organic conductors: structural aspects. Phys. Status Solidi B 2012, 249, 937-942.

22. Jacobsen, C.S.; Tanner, D.B.; Bechgaard, K. Optical and infrared properties of tetramethyltetraselenafulvalene $\left[(\mathrm{TMTSF})_{2} \mathrm{X}\right]$ and tetramethyltetrathiafulvalene [(TMTTF $\left.)_{2} \mathrm{X}\right]$ compounds. Phys. Rev. B 1983, 28, 7019-7032.

23. de Souza, M.; Hofmann, D.; Foury-Leylekian, P.; Moradpour, A.; Pouget, J.-P.; Lang, M. Exploring the charge-ordering transition in (TMTTF $)_{2} \mathrm{X}$ via thermal expansion measurements. Phys. B 2010, 405, S92-S94.

24. de Souza, M.; Foury-Leylekian, P.; Moradpour, A.; Pouget, J.-P.; Lang, M. Evidence for lattice effects at the charge-ordering transition in (TMTTF) 2 X. Phys. Rev. Lett. 2008, 101, 216403. 
25. Lang, M.; Müller, J.; Steglich, F.; Brühl, A.; Wolf, B.; Dressel, M. Anomalous thermal expansion behaviour in (TMTTF) $)_{2} \mathrm{PF}_{6}$ and $(\mathrm{TMTSF})_{2} \mathrm{PF}_{6}$ around $100 \mathrm{~K}$ : evidence for a strong spin-phonon interaction? J. Phys. IV France 2004, 114, 111-112.

26. Bechgaard, K.; Jacobsen, C.S.; Mortensen, K.; Pedersen, H.J.; Thorup, N. The properties of five highly conducting salts: (TMTSF) $2 \mathrm{X}, \mathrm{X}=\mathrm{PF}_{6}{ }^{-}, \mathrm{AsF}_{6}{ }^{-}, \mathrm{SbF}_{6}{ }^{-}, \mathrm{BF}_{4}{ }^{-}$and $\mathrm{NO}_{3}{ }^{-}$derived from tetramethyltetraselenafulvalene (TMTSF). Solid State Commun. 1980, 33, 1119-1125.

27. Yu, W.; Zhang, F.; Zamborszky, F.; Alavi, B.; Baur, A.; Merlic, C.A.; Brown, S.E. Electron-lattice coupling and broken symmetries of the molecular salt (TMTTF) ${ }_{2} \mathrm{SbF}_{6}$. Phys. Rev. B 2004, 70, 121101 (R).

28. Furukawa, K.; Hara, T.; Nakamura, T. Deuteration effect and possible origin of the charge-ordering transition of (TMTTF) $)_{2}$ X. J. Phys. Soc. Jpn. 2005, 74, 3288-3294.

29. Williams, J.M.; Beno, M.A.; Sullivan, J.C.; Banovetz, L.M.; Braam, J.M.; Blackman, G.S.; Carlson, C.D.; Greer, D.L.; Loesing, D.M.; Carneiro, K. The design of organic metals based on TMTSF and TMTTF: novel structural implications and predictions. J. Phys. (Paris) 1983, 44, C3-941-949.

30. Scott, J.C.; Pedersen, H.J.; Bechgaard, K. Proton NMR in the organic conductor tetramethyltetraselenafulvalenium hexafluorophosphate. Phys. Rev. B 1981, 24, 475-477.

31. McBrierty, V.J.; Douglass, D.C.; Wudl, F.; Aharon-Shalom, E. Nuclear resonance and relaxation in ditetramethyltetraselenafulvalenium salts. Phys. Rev. B 1982, 26, 4805-4809.

32. Scott, J.C.; Engler, E.M.; Clark, W.G.; Murayama, C.; Bechgaard, K.; Pedersen, H.J. NMR studies of (TMTSF) ${ }_{2} \mathrm{PF}_{6}$. Mol. Cryst. Liq. Cryst. 1982, 79, 61-65.

33. Stein, P.C.; Moradpour, A.; Jérome, D. Nuclear relaxation in tetramethyltetraselenafulvalene salts (TMTSF) $)_{2} \mathrm{X},\left(\mathrm{X}=\mathrm{PF}_{6}, \mathrm{ClO}_{4}\right)$. J. Phys. Lett. (Paris) 1985, 46, L241-L247.

34. Foury-Leylekian, P.; Petit, S.; André, G.; de Souza, M.; Lang, M.; Ressouche, E.; Moradpour, A.; Pouget, J.-P*. Low temperature structural ordering in the (TMTSF) ${ }_{2} \mathrm{PF}_{6}$ and $\mathrm{AsF}_{6}$ Bechgaard salts. In preparation, 2012.* Laboratoire de Physique des Solides, Université Paris-sud, 91405 Orsay, France.

35. Aoyagi, B.; Kato, K.; Ota, A.; Yamochi, A.; Saito, G.; Suematsu, H.; Sakata, M.; Takata, M. Direct Observation of Bonding and Charge Ordering in (EDO-TTF $)_{2} \mathrm{PF}_{6}$. Angew. Chem. Int. Ed. 2004, 43, 3670-3676.

36. Pouget, J.-P. Structural Instabilities. In Highly Conducting Quasi One Dimensional Organic Crystals; Conwell, E.M., Ed.; Academic Press: New York, NY, USA, 1988; Semiconductors and Semimetals Volume 27, pp. 87-215.

37. Shirane, G.; Shapiro, S.M.; Comès, R.; Garito, A.F.; Heeger, A.J. Phonon dispersion and Kohn anomaly in tetrathiafulvalene-tetracyanoquinodimethane (TTF-TCNQ). Phys. Rev. B 1976, 14, 2325-2334.

38. Pouget, J.P. Structural Instabilities of One-Dimensional Conductors. In Low-Dimensional Conductors and Superconductors; Jérome, D., Caron, L.G., Eds.; Plenum Press: New York, NY, USA, 1987; Volume 155, pp. 17-45. 
39. Ribault, M.; Pouget, J.-P.; Jérome, D.; Bechgaard, K. Superconductivity and Absence of a Kohn Anomaly in the Quasi One Dimensional Organic Conductor: (TMTSF) ${ }_{2} \mathrm{AsF}_{6}$. J. Phys. Lett. (Paris) 1980, 41, L607-L610.

40. Pouget, J.-P.; Moret, R.; Comès, R.; Bechgaard, K.; Fabre, J.M.; Giral, L. X-Ray Diffuse Scattering Study of Some (TMTSF) 2 X and (TMTTF) 2 X Salts. Mol. Cryst. Liq. Cryst. 1982, 79, 129-143.

41. Slater, J.C. Magnetic Effect and the Hartree-Fock Equation. Phys. Rev. 1951, 82, 538-541.

42. Pouget, J.-P. X-ray diffuse scattering as precursor of incommensurate Peierls transitions in one-dimensional organic charge transfer conductors. Z. Kristallogr. 2004, 219, 711-718.

43. Cao, N.; Timusk, T.; Bechgaard, K. Unconventional Electrodynamic Response of the Quasi-OneDimensional Organic Conductor (TMTSF) ${ }_{2} \mathrm{ClO}_{4}$. J. Phys. I France 1996, 6, 1719-1726.

44. Dressel, M.; Schwartz, A.; Grüner, G.; Degiorgi, L. Deviations from Drude Response in LowDimensional Metals: Electrodynamics of the Metallic State of (TMTSF) ${ }_{2} \mathrm{PF}_{6}$. Phys. Rev. Lett. 1996, 77, 398-401.

45. Schwartz, A.; Dressel, M.; Grüner, G.; Vescoli, V.; Degiorgi, L.; Giamarchi, T. On-chain electrodynamics of metallic (TMTSF $)_{2} \mathrm{X}$ salts: observation of Tomonaga-Luttinger liquid response. Phys. Rev. B 1998, 58, 1261-1271.

46. Wzietek, P.; Creuzet, F.; Bourbonnais, C.; Jérome, D.; Bechgaard, K.; Batail, P. Nuclear-relaxation and electronic correlations in quasi-one-dimensional organic conductors. 2. Experiments. J. Phys. I France 1993, 3, 171-201.

47. Chaikin, P.M.; Tiedje, T.; Bloch, A.N. Sound velocity measurements in (TMTSF) ${ }_{2} \mathrm{PF}_{6}$. Solid State Commun. 1982, 41, 739-742.

48. Shi, X.D.; Chiang, L.; Upasani, R.; Chaikin, P.M. Sound velocity studies of Bechgaard salts $(\mathrm{TMTSF})_{2} \mathrm{PF}_{6}$ and (TMTSF) ${ }_{2} \mathrm{ClO}_{4}$. In Advanced Organic Solid State Materials; Chiang, L.Y., Chaikin, P.M., Cowan, D.O., Eds.; Materials Research Society: Pittsburgh, PA, USA, 1990; pp. 239-244.

49. Fertey, P.; Poirier, M.; Batail, P. Microwave transport approach to the coherence of interchain hopping in (TMTSF) ${ }_{2} \mathrm{PF}_{6}$. Eur. Phys. J. B 1999, 10, 305-309.

50. Fertey, P.; Poirier, M.; Batail, P. H-T behaviors of the transverse microwave conductivity of (TMTSF) $)_{2}$ X crystals. Synth. Met. 1999, 103, 2076-2077.

51. Bourbonnais, C. Organic Superconductors: Reduced Dimensionality and Correlation Effects. Synth. Met. 1997, 84, 19-24.

52. Bourbonnais, C.; Jérome, D. Electronic confinement in organic metals. Science 1998, 281, 1155-1156.

53. Giamarchi, T. Theoretical Framework for Quasi-One Dimensional Systems. Chem. Rev. 2004, 104, 5037-5055.

54. Delrieu, J.M.; Roger, M.; Toffano, Z.; Moradpour, A.; Bechgaard, K. NMR proton lineshape in (TMTSF) $)_{2} \mathrm{X}$ - incommensurability of nesting wave vector and order parameter J. Phys. (Paris) 1986, 47, 839-861.

55. Takahashi, T.; Maniva, Y.; Kawamura, H.; Saito, G. Determination of SDW characteristics in (TMTSF) ${ }_{2} \mathrm{PF}_{6}$ by ${ }^{1} \mathrm{H}$ NMR analysis. J. Phys. Soc. Jpn. 1986, 55, 1364-1373. 
56. Ducasse, L.; Abderrabba, M.; Gallois, B. Temperature dependence of the Fermi surface topography in the (TMTSF) $)_{2} \mathrm{X}$ and (TMTTF $)_{2} \mathrm{X}$ families. J. Phys. C: Solid State Phys. 1985, 18 , L947-L951.

57. Vaca, P.; Coulon, C. Magnetic phase-transition in Bechgaard salts and related-compounds electronic localization and competing interchain couplings. Phase Transitions 1991, 30, 49-62.

58. Moret, R.; Pouget, J.P.; Comès, R.; Bechgaard, K. X-ray Scattering evidence for anion ordering and structural distortions in the low-temperature phase of Di (Tetramethyltetraselenafulvalenium) perrhenate [(TMTSF $)_{2} \mathrm{ReO}_{4}$ ]. Phys. Rev. Lett. 1982, 49, 1008-1012.

59. Rindorf, G.; Soling, H.; Thorup, N. Di(4,4',5,5'-tetramethyl-2,2'-bi-\&,3-diselenolyliden)ium Perrhenate, $\mathrm{C}_{20} \mathrm{H}_{24} \mathrm{Se}_{8}^{+} \cdot \mathrm{ReO}_{4}$, (TMTSF) ${ }_{2} \mathrm{ReO}_{4}$. Detailed superstructure at $120 \mathrm{~K}$. Acta Cryst. C 1984, 40, 1137-1139.

60. By the same argument one obtains a $3 \mathrm{D} 4 \mathrm{k}_{\mathrm{F}} \mathrm{CDW}$ or $\mathrm{CO}$ if the alternation of charge rich and charge poor molecules along a given stack is fixed by uniform shifts of anions separated by $\mathbf{a}$ and $\mathbf{d}_{\mathrm{i}}=\mathbf{a} \pm \mathbf{b}-\mathbf{c}$ ( \pm corresponding to the two coupling process considered in the text and in Figure 9). $\mathbf{q a}=\mathbf{q} \mathbf{d}_{\mathrm{i}}=0(\bmod .2 \pi)$ leads to $\mathrm{q}_{\mathrm{a}}=0$ and $\mathrm{q}_{\mathrm{a}} \pm \mathrm{q}_{\mathrm{b}}+\mathrm{qc}=0(\bmod .1)$. These relationships leads to $\pm q_{b}+q_{c}=0$ (mod.1) which has two solutions: $q_{b}=q c=0$ and $q_{b}=q c=1 / 2$. They corresponds respectively to the ferroelectric $\mathrm{q}_{\mathrm{F}}=(0,0,0)$ and antiferroelectric $\mathrm{q}_{\mathrm{AF}}=(0,1 / 2,1 / 2)$ CO patterns.

61. Pouget, J.-P.; Shirane, G.; Bechgaard, K.; Fabre, J.M. X-ray evidence of a structural phase transition in di-tetramethyltetraselenafulvalenium perchlorate [(TMTSF $\left.)_{2} \mathrm{ClO}_{4}\right]$, pristine and slightly doped. Phys. Rev. B 1983, 27, 5203-5206.

62. Pouget, J.-P.; Moret, R.; Comès, R.; Shirane, G.; Bechgaard, K.; Fabre, J.M. X-ray evidence of competing orderings in (TMTSF) ${ }_{2} \mathrm{ClO}_{4}$ and related alloys. J. Phys. (Paris) 1983, 44, C3-969-975.

63. Liu, Q.; Ravy, S.; Pouget, J.P.; Johannsen, I. Bechgaard, K. X-ray investigation of the tetramethyldithiadiselenafulvalene (TMDTDSF) ${ }_{2} \mathrm{X}$ series of organic conductors. II. Influence of the orientational disorder on the structural instabilities. J. Phys. I France 1993, 3, 821-837.

64. Liu, Q.; Ravy, S.; Pouget, J.P.; Johannsen, I.; Bechgaard, K. X-ray investigation of the tetramethyldithiadiselenafulvalene (TMDTDSF) ${ }_{2} \mathrm{X}$ series of organic conductors. I. Study of the orientational disorder of the TMDTDST molecule. J. Phys. I France 1993, 3, 803-819.

65. Pouget, J.-P.; Ravy, S. X-Ray evidence of charge density wave modulations in the magnetic phases of (TMTSF) ${ }_{2} \mathrm{PF}_{6}$ and (TMTTF) $)_{2}$ Br. Synth. Met. 1997, 85, 1523-1528.

66. Coulon, C.; Delhaes, P.; Flandrois, S.; Lagnier, R.; Bonjour, E.; Fabre, J.M. A new survey of the physical properties of the (TMTTF $)_{2} \mathrm{X}$ series. Role of the counterion ordering. J. Phys. (Paris) 1982, 43, 1059-1067.

67. Coulon, C.; Delhaes, P.; Amiell, J.; Manceau, J.P.; Fabre, J.M.; Giral, L. Effet of doping (TMTSF) ${ }_{2} \mathrm{ClO}_{4}$ with TMTTF I. Ambient pressure result: A competition between the different possible ground states. J. Phys. (Paris) 1982, 43, 1721-1729.

68. Foury-Leylekian, P.; Le Bolloc'h, D.; Hennion, B.; Ravy, S.; Moradpour, A.; Pouget, J.-P. Neutron-scattering evidence for a spin-Peierls ground state in (TMTTF) ${ }_{2} \mathrm{PF}_{6}$. Phys. Rev. B 2004, 70, 180405 (R).

69. Dumoulin, L.; Bourbonnais, C. Theory of lattice and electronic fluctuations in weakly localized spin-peierls systems. J. Phys. I France 1996, 6, 1727-1744. 
70. Liu, Q.; Ravy, S.; Pouget, J.-P.; Coulon, C.; Bourbonnais, C. Structural fluctuations and spinPeierls transitions revisited. Synth. Met. 1993, 56, 1840-1845.

71. Dumm, M.; Loidl, A.; Alavi, B.; Starkey, K.P.; Montgomery, L.K.; Dressel, M. Comprehensive ESR studies of the antiferromagnetic ground states in the one dimensional spin systems $(\mathrm{TMTSF})_{2} \mathrm{PF}_{6}$, (TMTSF $)_{2} \mathrm{AsF}_{6}$ and $(\mathrm{TMTTF})_{2} \mathrm{Br}$. Phys. Rev. B 2000, 62, 6512-6520 and references there in.

72. Pouget, J.-P. Microscopic interactions in $\mathrm{CuGeO}_{3}$ and organic Spin-Peierls systems deduced from their pretransitional fluctuations. Eur. Phys. J. B 2001, 20, 321-333 (Erratum Eur. Phys. J. B 2001, 24, 415).

73. Pouget, J.-P. Bond and charge ordering in low-dimensional organic conductors. Phys. B 2012, 407, 1762-1770.

74. Creuzet, F.; Bourbonnais, C.; Caron, L.G.; Jérome, D.; Bechgaard, K. A ${ }^{13}$ C NMR study of the interplay between the spin-Peierls and antiferromagnetic ground states in (TMTTF) ${ }_{2} \mathrm{PF}_{6}$ under pressure. Synth. Met. 1987, 19, 289-294.

75. Nakamura, T.; Nobutoki, T.; Kobayashi, Y.; Takahashi, T.; Saito, G. ${ }^{1}$ H-NMR investigation of the SDW wave-number in (TMTTF) ${ }_{2}$ Br. Synth. Met. 1995, 70, 1293-1294.

76. Seo, H.; Merino, J.; Yoshioka, H.; Ogata, M. Theoretical aspects of charge ordering in molecular conductors. J. Phys. Soc. Jpn. 2006, 75, 051009.

77. Zorina, L.; Simonov, S.; Mézière, C.; Canadell, E.; Suh, S.; Brown, S.E.; Foury-Leylekian, P.; Fertey, P.; Pouget, J.P.; Batail, P. Charge ordering, symmetry and electronic structures issues and Wigner crystal structure of the quarter-filled band Mott insulators and high pressure metals $\delta$-(EDT-TTF-CONMe $)_{2} \mathrm{X}, \mathrm{X}=\mathrm{Br}$ and $\mathrm{AsF}_{6}$. J. Mat. Chem. 2009, 19, 6980-6994.

78. Tomic, S.; Biskup, N.; Babic, S.D.; Maki, K. Commensurate spin-density-wave state in (TMTTF) $)_{2} \mathrm{Br}$ - single particle and collective charge dynamics. Europhys. Lett. 1994, 26, 295-301.

79. Ashikawa, A.; Matsunaga, N.; Nomura, K.; Nakamura, T.; Takahashi, T.; Saito, G. Pressure and Magnetic Field Dependence of SDW transition in (TMTTF) ${ }_{2}$ Br. Phys. Stat. Sol. B 2001, 223, 539-543.

80. Fujiyama, S.; Nakamura, T. NMR studies of the localized states of (TMTTF) ${ }_{2}$ Br. J. Phys. Chem. Solids 2002, 63, 1259-1261.

81. Javadi, H.H.S.; Laversanne, R.; Epstein, A.J. Microwave conductivity and dielectric constant of tetramethyltetrathiafulvalene salts $\left[(\mathrm{TMTTF})_{2} \mathrm{X}, \mathrm{X}=\mathrm{SCN}, \mathrm{ReO}_{4}, \mathrm{SbF}_{6}\right.$ ]. Phys. Rev. B 1988, 37, 4280-4283.

82. Nad, F.; Monceau, P.; Fabre, J.M. Low frequency dielectric permittivity of quasi-onedimensional conductor (TMTTF) ${ }_{2}$ Br. Eur. Phys. J. B 1998, 3, 301-306.

83. Clark, W.G.; Hanson, M.E.; Wong, W.H.; Alavi, B. Evidence that the SDW transition in (TMTSF) ${ }_{2} \mathrm{PF}_{6}$ is first order. Phys. B 1994, 194-196, 285-286.

84. Kagoshima, S.; Saso, Y.; Maesato, M.; Kondo, R.; Hasegawa, T. Low temperature diffuse X-ray studies of charge-density waves coexisting with spin-density waves in the organic conductors (TMTSF $)_{2} \mathrm{PF}_{6}$ and (TMTSF) ${ }_{2} \mathrm{AsF}_{6}$. Solid State Commun. 1999, 110, 479-483.

85. Gufan, Yu, M.; Larin, E.S. Theory of phase transitions described by two order parameters. Sov. Phys. Solid State 1980, 22, 270-275. 
86. Takahashi, T.; Maniwa, Y.; Kawamura, H.; Saito, G. Determination of SDW characteristics in (TMTSF) $)_{2} \mathrm{PF}_{6}$ by ${ }^{1} \mathrm{H}-\mathrm{NMR}$ analysis. Phys. B 1986, 143, 417-421.

87. Takahashi, T.; Maniwa, Y.; Kawamura, H.; Murata, K.; Saito, G. NMR analysis of electronic properties in organic superconductors (TMTSF) $)_{2} \mathrm{PF}_{6}$ and $\beta$-(BEDT-TTF) $)_{2} \mathrm{I}_{3}$. Synth. Met. 1987, 19, 225-230.

88. Nickel, J.C.; Duprat, R.; Bourbonnais, C.; Dupuis, N. Triplet Superconductivity Pairing and Density Wave Instabilities in Organic Conductors. Phys. Rev. Lett. 2005, 95, 247001.

89. Nickel, J.C.; Duprat, R.; Bourbonnais, C.; Dupuis, N. Superconductivity pairing and density wave instabilities in quasi-one-dimensional conductors. Phys. Rev. B 2006, 73, 165126.

90. Kuroki, K. Pairing symmetry considerations in organic superconductors. J. Phys. Soc. Jpn. 2006, $75,051013$.

91. Ng, H.K.; Timusk, T.; Bechgaard, K. Far infra-red studies of bis (tetramethyltetraselenafulvalene) hexafluoroantimonate [(TMTSF $\left.)_{2} \mathrm{SbF}_{6}\right]$ : Coexistence of metallic and semiconducting states. Phys. Rev. B 1984, 30, 5842-5846.

92. Grüner, G. The dynamics of spin-density waves. Rev. Mod. Phys. 1994, 66, 1-24.

93. Laversanne, R.; Coulon, C.; Gallois, B.; Pouget, J.P.; Moret, R. Structural and electrical properties of (TMTTF $)_{2} \mathrm{MF}_{6}$ salts $(\mathrm{M}=\mathrm{P}, \mathrm{As}, \mathrm{Sb})$. Role of the anions. J. Phys. Lett. (Paris) 1984, 45, L393-L399.

94. Coulon, C.; Maaroufi, A.; Amiell, J.; Dupart, E.; Flandrois, S.; Delhaes, P.; Moret, R.; Pouget, J.P.; Morand, J.P. Antiferromagnetic and structural instabilities in tetramethyltetrathiafulvalene thiocyanate [(TMTTF $)_{2}$ SCN]. Phys. Rev. B 1982, 26, 6322-6325.

95. Coulon, C.; Parkin, S.S.P.; Laversanne, R. Structureless transition and strong localization effects in bis-tetramethyltetrathiafulvalenium salts $\left[(\mathrm{TMTTF})_{2} \mathrm{X}\right]$. Phys. Rev. B 1985, 31, 3583-3587.

96. Brown, S.E.; Javadi, H.H.S.; Laversanne, R. Sound Velocity Measurements in (TMTTF) 2 X Salts, $\mathrm{X}=\mathrm{ReO}_{4}, \mathrm{AsF}_{6}, \mathrm{SbF}_{6}$. In Advanced Organic Solid State Materials; Chiang, L.Y., Chaikin, P.M., Cowan, D.O., Eds.; Materials Research Society: Pittsburgh, PA, USA, 1990; pp. 245-250.

97. Chow, D.S.; Zamborsky, F.; Alavi, B.; Tantillo, D.J.; Baur, A.; Merlic, C.A.; Brown, S.E. Charge ordering in the TMTTF family of molecular conductors. Phys. Rev. Lett. 2000, 85, 1698-1701.

98. Dumm, M.; Salameh, B.; Abaker, M.; Montgomery, L.K.; Dressel, M. Magnetic and optical studies of spin and charge ordering in (TMTTF) ${ }_{2} \mathrm{AsF}_{6}$. J. Phys. IV (France) 2004, 114, 57-60.

99. Dumm, M.; Abaker, M.; Dressel, M.; Montgomery, L.K. Charge order in (TMTTF) ${ }_{2} \mathrm{PF}_{6}$ investigated by infrared spectroscopy. J. Low. Temp. Phys. 2006, 142, 609-612.

100. Monceau, P.; Nad, F.; Brazovski, S. Ferroelectric Mott-Hubbard phase of organic (TMTTF) ${ }_{2} \mathrm{X}$ conductors. Phys. Rev. Lett. 2001, 86, 4080-4083.

101. Foury-Leylekian, P.; Ravy, S.; Pouget, J.-P. Stuctural properties of strongly correlated quasi-1D organic systems. Phys. B 2002, 312-313, 574-577.

102. Ravy, S.; Foury-Leylekian, P.; Le Bolloc'h, D.; Pouget, J.-P.; Fabre, J.M.; Prado, R.J.; Lagarde, P. Structural instability and electronic localization in the 2/1 salts: The case of the Fabre and the (DMtTTF) ${ }_{2} \mathrm{ClO}_{4}$ salts. J. Phys. (Paris) IV 2004, 114, 81-85. 
103. Nogami, Y.; Nakamura, T. X-ray observation of $2 \mathrm{k}_{\mathrm{F}}$ and $4 \mathrm{k}_{\mathrm{F}}$ charge orderings in $(\mathrm{TMTTF})_{2} \mathrm{ReO}_{4}$ and (TMTTF) $)_{2} \mathrm{SCN}$ associated with anion orderings. J. Phys. (Paris) IV 2002, 12, Pr9-145-148.

104. Coulon, C.; Lalet, G.; Pouget, J.-P.; Foury-Leylekian, P.; Moradpour, A.; Fabre, J.M. Anisotropic conductivity and charge ordering in (TMTTF) $)_{2} \mathrm{X}$ salts probed by ESR. Phys. Rev. B 2007, 76, 085126.

105. Foury-Leylekian, P.; Petit, S.; Andre, G.; Moradpour, A.; Pouget, J.P. Neutron scattering evidence for a lattice displacement at the charge ordering transition of $(\mathrm{TMTTF})_{2} \mathrm{PF}_{6}$. Phys. B 2010, 405, S95-S97.

106. Granier, T.; Gallois, B.; Ducasse, L.; Fritsch, A.; Filhol, A. 4K crystallographic and electronic structures of (TMTTF) $)_{2} \mathrm{X}$ salts $\left(\mathrm{X}^{-}: \mathrm{PF}_{6}{ }^{-} ; \mathrm{AsF}_{6}{ }^{-}\right)$. Synth. Met. 1988, 24, 343-356.

107. Sawa, H. Direct Observation of Electronic State of (TMTTF) $)_{2} \mathrm{PF}_{6}$ Using Synchrotron Radiation X-ray Analysis. In Proceedings of International Research School and Workshop on Electronic Crystals; ECRYS: Cargèse, France, 15-27 August 2011.

108. In this respect the analysis performed in [105] and which considers only the shift of the anion sublattice is incomplete. As the neutron scattering length is similar for all the nucleus of (TMTTF $)_{2} \mathrm{PF}_{6}$, the 3 contributions given in the text have to be treated on equal footing in the structural refinement. Note that in deuterated samples the neutron scattering could be particularly sensitive to the deformation of the methyl group cavity at $T_{\mathrm{CO}}$.

109. Dressel, M.; Dumm, M.; Knoblauch, T.; Masimo, M. Comprehensive optical investigation of Charge Order in Organic chain compounds (TMTTF) $)_{2}$ X. Crystals 2012, in press.

110. Rose, E.; Dressel, M. Coupling between molecular chains and anions in (TMTTF) $)_{2} \mathrm{X}$ salts. Phys. B 2012, 407, 1787-1892.

111. Riera, J.; Poilblanc, D. Influence of the anion potential on the charge ordering in quasi-one-dimensional charge-transfer salts. Phys. Rev. B 2001, 63, 241102(R).

112. Pouget, J.-P.; Foury-Leylekian, P.; Le Bolloc'h, D.; Hennion, B.; Ravy, S.; Coulon, C.; Cardoso, V.; Moradpour, A. Neutron-Scattering Evidence for a Spin-Peierls ground State in (TMTTF $)_{2} \mathrm{PF}_{6}$. J. Low. Temp. Phys. 2006, 142, 147-152.

113. Köhler, B.; Rose, E.; Dumm, M.; Untereiner, G.; Dressel, M. Comprehensive transport study of anisotropy and ordering phenomena in quasi-one-dimensional (TMTTF $)_{2} \mathrm{X}$ salts $\left(\mathrm{X}=\mathrm{PF}_{6}, \mathrm{AsF}_{6}\right.$, $\mathrm{SbF}_{6}, \mathrm{BF}_{4}, \mathrm{ClO}_{4}, \mathrm{ReO}_{4}$ ). Phys. Rev. B 2011, 84, 035124.

114. Nad, F.; Monceau, P.; Nakamura, T.; Furukawa, K. The effect of deuteration on the transition into a charge ordered state of (TMTTF) $)_{2}$ X salts. J. Phys.: Condens. Matter 2005, 35, L399-L406.

115. Moret, R.; Pouget, J.P.; Comès, R.; Bechgaard, K. X-ray study of the anion ordering transition in di(tetramethyltetraselenafulvalen)-ium perchlorate (TMTSF) ${ }_{2} \mathrm{ClO}_{4}$ : quenching and irradiation effects. J. Phys. (Paris) 1985, 46, 1521-1732.

116. Zamborszky, F.; Yu, W.; Raas, W.; Brown, S.E.; Alavi, B.; Merlic, C.A.; Baur, A. Competition and coexistence of bond and charge orders in (TMTTF) ${ }_{2} \mathrm{AsF}_{6}$. Phys. Rev. B 2002, 66, 081103(R).

117. Nad, F.; Monceau, P. Dielectric Response of the Charge Ordered State in Quasi-One Dimensional Organic Conductors. J. Phys. Soc. Jpn. 2006, 75, 051005. 
118. Coulon, C.; Foury-Leylekian, P.; Fabre, J.M.; Pouget, J.-P. Electronic instabilities and irradiation effects in the (TMTTF $)_{2} \mathrm{X}$ series. Centre de Recherche Paul Pascal, Université Bordeaux I, France. Unpublished work, 2012.

119. Brazovskii, S. Ferroelectricity and Charge Ordering in Quasi-1D Organics Conductors. In The Physics of Organic Superconductors and Conductors; Lebed, A., Ed.; Springer-Verlag: Berlin, Germany, 2008; pp. 313-355.

120. Kirova, N.; Brazovskii, S. Ferroelectricity: From organic conductors to electronic polymers. Phys. B 2009, 404, 382-384.

121. Yamamoto, K.; Iwai, S.; Boyko, S.; Kashiwazaki, A.; Hiramatsu, F.; Okabe, C.; Nishi, N.; Yakushi, K. Strong optical nonlinearity and its ultrafast response associated with electron ferroelectricity in an organic conductor. J. Phys. Soc. Jpn. 2008, 77, 074709.

122. Alemany, P.; Pouget, J.-P.; Canadell, E. Essential role of anions in the charge ordering transition of $\alpha$-(BEDT-TTF) $)_{2} \mathrm{I}_{3}$. Phys. Rev. B 2012, 85, 195118.

123. Pouget, J.-P.; Khanna, S.K.; Denoyer, F.; Comès, R.; Garito, A.F.; Heeger, A.J. X-ray Observation of $2 \mathrm{k}_{\mathrm{F}}$ and $4 \mathrm{k}_{\mathrm{F}}$ Scatterings in Tetrathiafulvalene-Tetracyanoquinodimethane (TTF-TCNQ). Phys. Rev. Lett. 1976, 37, 437-440.

124. Nogami, Y.; Moret, R.; Pouget, J.-P.; Yamamoto, Y.; Oshima, K.; Hiraki, K.; Kanoda, K. X-ray structural study of $4 \mathrm{k}_{\mathrm{F}}$ Superlattice in (DMDCNQI) $)_{2}$ Ag. Synth. Met. 1999, 102, 1778.

125. Foury-Leylekian, P.; Auban-Senzier, P.; Coulon, C.; Jeannin, O.; Fourmigué, M.; Pasquier, C.; Pouget, J.-P. Phase diagram of the correlated quarter-filled-band organic salt series (o-DMTTF) $)_{2} \mathrm{X}$ (X=Cl, Br, I). Phys. Rev. B 1994, 50, 7131-7139.

126. Zamborszky, F.; Yu, W.; Raas, W.; Brown, S.E.; Alavi, B.; Merlic, C.A.; Baur, A.; Lefebvre, S.; Wzieteck, P. Influence of charge order on the ground state of TMTTF conductors. J. Phys. (Paris) IV 2002, 12, Pr9-139-144.

127. In contrast ${ }^{75} \mathrm{As}$ NMR $\mathrm{T}_{1}{ }^{-1}$ does not exhibit a divergence at $T_{\mathrm{CO}}$ but only a change of slope. This result could be understood if $\mathrm{AsF}_{6}$ plays a weaker role in the $\mathrm{CO}$ transition (through process 1) than in the SP transition (through process 2).

128. de Souza, M.; Brühl, A.; Müller, J.; Foury-Leylekian, P.; Moradpour, A.; Pouget, J.-P.; Lang, M. Thermodynamic studies at the charge ordering and spin-Peierls transitions in (TMTTF) $)_{2} \mathrm{X}$. Phys. B 2009, 404, 494-498.

129. Dumm, M.; Loidl, A.; Fravel, B.W.; Starkey, K.P.; Montgomery, L.K.; Dressel, M. Electron spin resonance studies on the organic linear chain compounds $(\mathrm{TMTCF})_{2} \mathrm{X}\left(\mathrm{C}=\mathrm{S}, \mathrm{Se} ; \mathrm{X}=\mathrm{PF}_{6}, \mathrm{AsF}_{6}\right.$, $\left.\mathrm{ClO}_{4}, \mathrm{Br}\right)$ Phys. Rev. B 2001, 61, 511-521.

130. Salameh, B.; Yasin, S.; Dumm, M.; Untereiner, G.; Montgomery, L.; Dressel, M. Spin dynamics of the organic linear chain compounds (TMTTF $)_{2} \mathrm{X}\left(\mathrm{X}=\mathrm{SbF}_{6}, \mathrm{AsF}_{6}, \mathrm{BF}_{4}, \mathrm{ReO}_{4}\right.$, and $\left.\mathrm{SCN}\right)$. Phys. Rev. B 2011, 83, 205126.

131. Foury-Leylekian, P.; Petit, S.; Coulon, C.; Hennion, B.; Moradpour, A.; Pouget, J.-P. Inelastic neutron scattering investigation of the magnetic excitations in the spin-Peierls ground state of (TMTTF $)_{2} \mathrm{PF}_{6}$. Phys. B 2009, 404, 537-540. 
132. Langlois, A.; Poirier, M.; Bourbonnais, C.; Foury-Leylekian, P.; Moradpour, A.; Pouget J.-P. Microwave dielectric study of spin-Peierls and charge ordering transitions in (TMTTF) ${ }_{2} \mathrm{PF}_{6} \mathrm{Salts}_{\text {. }}$ Phys. Rev. B 2010, 81, 125101.

133. Brown, S.E.; Clark, W.G.; Zamborszky, F.; Klemme, B.J.; Kriza, G.; Alavi, B.; Merlic, C.; Kuhns, P.; Moulton, W. ${ }^{13} \mathrm{C}$ NMR measurements of the high-magnetic-field, low-temperature phases of (TMTTF $)_{2} \mathrm{PF}_{6}$. Phys. Rev. Lett. 1998, 80, 5429-5432.

134. Brown, S.E.; Clark, W.G.; Alavi, B.; Hall, D.; Naughton, M.J.; Tantillo, D.J.; Merlic, C.A. High-field magnetization of the spin-Peierls compound (TMTTF) ${ }_{2} \mathrm{PF}_{6}$. Phys. Rev. B 1999, 60, 6270-6272.

135. Hirsch, J.E.; Scalapino, D.J. $2 \mathrm{p}_{\mathrm{F}}$ and $4 \mathrm{p}_{\mathrm{F}}$ instabilities in the one-quarter-filled-band Hubbard model. Phys. Rev. B 1983, 27, 7169-7185.

136. Hirsch, J.E.; Scalapino, D.J. $2 \mathrm{p}_{\mathrm{F}}$ and $4 \mathrm{p}_{\mathrm{F}}$ instabilities in the one dimensional Hubbard model. Phys. Rev. B 1984, 29, 5554-5561.

137. Ilakovac, V.; Ravy, S.; Pouget, J.P.; Lenoir, C.; Boubekeur, K.; Batail, P.; Dolanski Babic, S.; Biskup, N.; Korin-Hamzic, B.; Tomic, S.; Bourbonnais, C. Enhanced charge localization in the organic alloys [(TMTSF $\left.)_{1-\mathrm{x}}(\mathrm{TMTTF})_{\mathrm{x}}\right]_{2} \mathrm{ReO}_{4}$. Phys. Rev. B 2011, 84, 195134.

138. The assertion done in [137] that the inversion symmetry is broken on the anion sites at RT above the AO transition is incorrect. Only inversion centers located on the organic stacks between the donors are removed by the chemical CO.

139. Chow, D.S.; Wzietek, P.; Fogliatti, D.; Alavi, B.; Tantilo, D.J.; Merlic, C.A.; Brown, S.E. Singular behavior in the pressure-tuned competition between spin-Peierls and antiferromagnetic ground states of (TMTTF) ${ }_{2} \mathrm{PF}_{6}$. Phys. Rev. Lett. 1998, 81, 3984-3987.

140. Rose, E.; Loose, C.; Kortus, J.; Pashkin, A.; Kuntscher, C.A.; Ebbinghaus, S.G.; Hanfland, M.; Lissner, F.; Schleid, Th.; Dressel, M. Pressure dependence of Crystal Structure and Electronic Band Structure of (TMTTF) ${ }_{2} \mathrm{PF}_{6}$. J. Phys.: Condens. Matter 2012, in press.

141. Le Pévelen, D.; Gaultier, J.; Barrens, Y.; Chasseau, D.; Castet, F.; Ducasse, L. Temperature and pressure dependencies of the crystal structure of the organic superconductor (TMTSF) ${ }_{2} \mathrm{ClO}_{4}$. Eur. Phys. J. B 2001, 19, 363-373.

142. Jacobsen, C.S.; Pedersen, H.J.; Mortensen, K.; Rindorf, G.; Thorup, N.; Torrance, J.B.; Bechgaard, K. An unusual metal-insulator transition: bis(tetramethyltetraselenafulvalenium)perrhenate (TMTSF) ${ }_{2} \mathrm{ReO}_{4}$. J. Phys. C: Solid State Phys. 1982, 15, 2651-2663.

143. Pouget, J.-P.; Moret, R.; Comès, R.; Bechgaard, K. X-ray diffuse scattering study of superstructure formation in tetramethyltetraselenafulvalenium perrhenate (TMTSF) ${ }_{2} \mathrm{ReO}_{4}$ and nitrate (TMTSF) ${ }_{2} \mathrm{NO}_{3}$. J. Phys. Lett. (Paris) 1981, 42, L543-L546.

144. Grant, P.M. Broken-Symmetry Band Structure of Ditetramethyltetradelenafulvalene-X [(TMTSF $\left.)_{2} \mathrm{X}\right]$. Phys. Rev. Lett. 1983, 50, 1005-1008.

145. Grant, P.M. Electronic structure of the 2:1 charge transfer salts of TMTCF. J. Phys. (Paris) 1983, 44, C3-847-857.

146. Emery, V.J. Some basic questions in organic superconductivity. J. Phys. (Paris) 1983, 44, C3-977-982. 
147. Brazovslii, S.; Yakovenko, V. On the theory of phase transitions in organic superconductors. J. Phys. Lett. (Paris) 1985, 46, L111-L116.

148. Bruinsma, R.; Emery, V.J. Theory of anion ordering in TMTSF compounds. J. Phys. (Paris) 1983, 44, C3-1115-1120.

149. Emge, T.J.; Wang, M.H.; Beno, M.A.; Williams, J.M.; Whangbo, M.H.; Evain, M. Effect of anion ordering on the $\mathrm{H}$-anion interaction and band electronic structure of $(\mathrm{TMTSF})_{2} \mathrm{BF}_{4}$ at $20 \mathrm{~K}$. J. Am. Chem. Soc. 1986, 108, 8215-8223.

150. Ducasse, L.; Abderrabba, M.; Gallois, B.; Chasseau, D. Influence of temperature on the band structures of (TMTSF) $)_{2} \mathrm{X}$ and (TMTTF) $)_{2} \mathrm{X}\left(\mathrm{X}^{-}=\right.$tetrahedral anion). Synth. Met. 1987, 19, 327-332.

151. Tomic, S.; Jérome, D.; Bechgaard, K. Influence of the cooling rate on the ground state of the organic conductor (TMTSF) ${ }_{2} \mathrm{ReO}_{4}$. J. Phys. C: Solid State Phys. 1984, L11-L16.

152. Moret, R.; Ravy, S.; Pouget, J.P.; Comès, R.; Bechgaard, K. Anion-Ordering Phase Diagram of di(tetramethyltetraselenafulvalenium)-Perrhenate, (TMTSF) ${ }_{2} \mathrm{ReO}_{4}$. Phys. Rev. Lett. 1986, 57, 1915-1918.

153. Jo, Y.J.; Kang, H.; Kang, W. Review of the P-T phase diagram of the organic superconductor (TMTSF) ${ }_{2} \mathrm{ReO}_{4}$. Synth. Met. 2001, 120, 1043-1044.

154. Nakamura, T.; Furukawa, K.; Hara, T. ${ }^{13} \mathrm{C}$ NMR Analyses of Successive Charge Ordering in (TMTTF $)_{2} \mathrm{ReO}_{4}$. J. Phys. Soc. Jpn. 2006, 75, 013707.

155. Nogami, Y.; Ito, T.; Yamamoto, K.; Irie, N.; Horita, S.; Kambe, T.; Nagao, N.; Oshima, K.; Ikeda, N.; Nakamura, T. X-ray structural study of charge and anion orderings of TMTTF salts. $J$. Phys. (Paris) IV 2005, 131, 39-42.

156. Ravy, S.; Pouget, J.-P.; Moret, R.; Wudl, F. Successive phase transitions in the organic conductor (TMTSF) ${ }_{2} \mathrm{PF}_{2} \mathrm{O}_{2}$. J. Phys. I France 1991, 1, 703-720.

157. Moret, R.; Pouget, J.-P.; Comès, R.; Bechgaard, K. Structural phase transitions in the (TMTSF) 2 X and (TMTTF) $)_{2} \mathrm{X}$ series: A survey with some new results. J. Phys. (Paris) 1983, 44, C3-957-962.

158. Yamaura, J.; Jo, Y.J.; Kang, H.; Chung, O.H.; Kang, W. X-ray study at low temperature and under pressure in (TMTSF) ${ }_{2} \mathrm{FSO}_{3}$. Unpublished work, 2005.

159. Lacoe, R.C.; Wolf, S.A.; Chaikin, P.M.; Wudl, F.; Aharon-Shalom, E. Metal-insulator transitions and superconductivity in ditetramethyltetraselenafulvalenium fluorosulfonate [(TMTSF $)_{2} \mathrm{FSO}_{3}$ ]. Phys. Rev. B 1983, 27, 1947-1950.

160. Jo, Y.J.; Choi, E.S.; Kang, H.; Kang, W.; Seo, I.S.; Chung, O.H. Refinement of the pressuretemperature phase diagram of the organic superconductor with asymmetric anion (TMTSF) ${ }_{2} \mathrm{FSO}_{3}$. Phys. Rev. B 2003, 67, 014516.

161. Takahashi, T. Charge fluctuation, charge ordering, and zero-gap state in organic conductors. Phys. B 2012, 407, 1757-1761.

162. Alemany, P.; Canadell, E.; Pouget, J.-P. Ab-initio electronic structure of (TMTSF) ${ }_{2} \mathrm{ClO}_{4}$. Institut de Ciència de Materials de Barcelona, Bellaterra, Spain, Unpublished work, 2012.

163. Nagai, Y.; Nakamura, H.; Machida, M. Superconducting gap function in the organic superconductor (TMTSF) ${ }_{2} \mathrm{ClO}_{4}$ with anion ordering; First-principles calculations and quasiclassical analysis for angle-resolved heat capacity. Phys. Rev. B 2011, 83, 104523. 
164. Pouget, J.-P.; Kagoshima, S.; Tamegai, T.; Nogami, Y.; Kubo, K.; Nakajima, T.; Bechgaard, K. High resolution $\mathrm{x}$-ray scattering study of the anion ordering phase transition of (TMTSF) ${ }_{2} \mathrm{ClO}_{4}$. J. Phys. Soc. Jpn. 1990, 59, 2036-2053.

165. Barrens, Y.; Gaultier, J.; Brachetti, S.; Guionneau, P.; Chasseau, D.; Fabre, J.M. Low temperature structural properties of the (TMTSF) ${ }_{2} \mathrm{NO}_{3}$ salt. Synth. Met. 1999, 103, 2042-2043.

166. Matsunaga, N.; Ishikawa, A.; Hoshikawa, A.; Nomura, K.; Takasaki, S.; Yamada, J.; Nakatsuji, S.; Anzai, H. Anion disorder and two-dimensionality in the superconducting and SDW states of (TMTSF) ${ }_{2} \mathrm{ClO}_{4}$. J. Low Temp. Phys. 1999, 117, 1735-1739.

167. In the case of a random distribution of domain sizes, the probability that two sites separated by $x$ belong to the same domain is given by $\exp -\mu \mathrm{x}$, where $\mu$ is the constant probability to cross a domain wall per unit length. The 3D Fourier transform of this probability function, assuming $\mu$ isotropic, is a Lorentzian squared function, whose HWHM $\Delta \mathrm{q}$ is linearly related to $\mu$ by $\Delta \mathrm{q} \approx$ $0.64 \mu$. $\mu$ is connected to the linear density of domain walls along the direction of measurement of $\Delta q . \mu$ should also depend upon the geometry the domain pattern.

168. The average domain size $\mathrm{L}$ in a given direction is inversely proportional to the HWHM $\Delta \mathrm{q}$ of the reflection (expressed in $\AA^{-1}$ ), measured in this direction. In a general manner the profile of the reflection is the Fourier transform of the average shape of the ordered domain. $\mathrm{L}$ is given by the relationship $\mathrm{L} \approx \mathrm{C}(\mathrm{d}) / \Delta \mathrm{q}$, where the constant $\mathrm{C}(\mathrm{d})$ depends upon the dimension, $\mathrm{d}$, of independent directions involved in the Fourier transform. For a segment of length $\mathrm{L}$ (or along one edge of a parallelepiped): $\mathrm{C}(1) \approx 2.79$; for a disc of diameter $\mathrm{L}: \mathrm{C}(2) \approx 3.2$, and for a sphere of diameter $\mathrm{L}$ : $\mathrm{C}(3) \approx 3.72$. L depends weakly of the shape of the domain. In [164], L is obtained using C(1).

169. Haddad, S.; Charfi-Kaddour, S.; Pouget, J.-P. Inhomogeneous superconductivity in organic conductors: the role of disorder and magnetic field. J. Phys.: Condens. Matter 2011, 23, 464205.

170. Ilakovac, V.; Ravy, S.; Boubekeur, K.; Lenoir, C.; Batail, P.; Pouget, J.-P. Substitutional disorder and anion ordering transition in the (TMTSF $)_{2}\left(\mathrm{ReO}_{4}\right)_{1-x}\left(\mathrm{ClO}_{4}\right)_{x}$ solid solution. Phys. Rev. B 1997, $56,13878-13887$.

171. $\mu^{-1}$, which is quasi-isotropic, amounts to $400 \AA$ in relaxed samples and decreases rapidly to $25 \AA$ / $20 \AA$ for $x=5 \% / 7 \%$ of $\mathrm{ReO}_{4}$. Interestingly for these last two concentrations, $\mu^{-1}$ amounts to the average distance between $\mathrm{ReO}_{4}$ substituent ( $24 \AA$ and $21 \AA$ respectively).

172. In this respect one observes at low temperature for $x=7 \%$ of $\mathrm{ReO}_{4}$ a coexistence of $(0,1 / 2,0)$ $\mathrm{AO}$ and $(1 / 2,1 / 2,1 / 2) \mathrm{AO}$ domains in close contact and whose diffraction pattern is a superimposition of LS shaped superlattice reflections [170]. As there is a random distribution of the two species of anion, $(1 / 2,1 / 2,1 / 2) \mathrm{AO}$ domains are nucleated in regions of excess of $\mathrm{ReO}_{4}$ (with respect to the average concentration) while $(0,1 / 2,0) \mathrm{AO}$ domains are nucleated in regions deficient in $\mathrm{ReO}_{4}$.

173. Joo, N.; Auban-Senzier, P.; Pasquier, C.R.; Monod, P.; Jérome, D.; Bechgaard, K. Supression of superconductivity by non-magnetic disorder in the organic superconductor $(\text { TMTSF })_{2}\left(\mathrm{ClO}_{4}\right)_{1-x}\left(\mathrm{ReO}_{4}\right)_{x}$. Eur. Phys. J. B 2004, 40, 43-48.

174. Joo, N.; Auban-Senzier, P.; Pasquier, C.R.; Jérome, D.; Bechgaard, K. Impurity-controlled superconductivity/spin density wave interplay in the organic superconductor (TMTSF) ${ }_{2} \mathrm{ClO}_{4}$. Europhys. Lett. 2005, 72, 645-651. 
175. Fertey, P.; Sayetat, F.; Muller, J.; Pouget, J.-P.; Lenoir, C.; Batail, P. Thermal behaviour of (TMTSF $)_{2} \mathrm{ClO}_{4}$ lattice parameters in the range 10K-300K. Physica C 1994, 235-240, 2459-2460.

176. Fertey, P.; Canadell, E.; Pouget, J.-P.; Sayetat, F.; Lenoir, C.; Batail, P; Muller, J. Cooling rate dependence of the lattice parameters of (TMTSF) $)_{2} \mathrm{ClO}_{4}$. Synth. Met. 1995, 70, 761-762.

177. Nakamura, T.; Kinami, R.; Takahashi, T.; Saito, G. ${ }^{1} \mathrm{H}$ NMR Study of the Magnetic Structure in (TMTTF) ${ }_{2}$ SCN. Synth. Met. 1997, 86, 2053-2054.

178. Parkin, S.S.P.; Coulon, C.; Moret, R.; Pouget, J.-P. Incommensurate structural modulation and electronic localization in bis-tetramethyltetraselenafulvalene thiocyanate. Phys. Rev. B 1987, 36, 2246-2250.

179. Kang, W.; Chung, O.H. Quasi-one-dimensional Fermi surface of (TMTSF) ${ }_{2} \mathrm{NO}_{3}$. Phys. Rev. $B$ 2009, 79, 045115.

180. Hiraki, K.; Nemoto, T.; Takahashi, T.; Kang, K.; Jo, Y.; Kang, W.; Cung, O.-H. NMR studies of the exotic members of the Bechgaard salts, $\mathrm{NO}_{3}$ and $\mathrm{FSO}_{3}$ salts. Synth. Met. 2003, 135-136, 691-692.

181. Poirier, M.; Langlois, A.; Bourbonnais, C.; Foury-Leylekian, P.; Moradpour, A.; Pouget, J.-P. Magneto-elastic coupling in the spin-Peierls ground state of hydrogenated and deuterated $(\mathrm{TMTTF})_{2} \mathrm{PF}_{6}$ salts. Departement de Physique, Université de Sherbrooke, Shergrooke, Canada.Unpublished work, 2012.

182. Pasquier, C.R.; Kang, N.; Salameh, B.; Auban-Senzier, P.; Jérome, D.; Brazovskii, S. Evolution of the spin-density wave-superconductivity texture in the organic superconductor (TMTSF) ${ }_{2} \mathrm{PF}_{6}$ under pressure. Phys. B 2012, 407, 1806-1809 and references there in.

183. Giovannetti, G.; Kumar, S.; Pouget, J.-P.; Cappone, M. Magnetic and charge orderings in predicting multiferroicity in $\mathrm{TMTTF}_{2}-\mathrm{PF}_{6}$ organic crystals. Unpublished work, 2012.

184. Khomskii, D.I. Multiferroics: Different ways to combine magnetism and ferroelectricity. J. Magn. Magn. Mater. 2006, 306, 1-8.

185. van den Brink, J.; Khomskii, D.I. Multiferroicity due to charge ordering. J. Phys.: Condens. Matter 2008, 20, 434217.

186. Holakovský, J. A New Type of Ferroelectric Phase Transition. Phys. Stat. Sol. B 1973, 56, 615-619.

187. Imry, Y. On the statistical mechanics of coupled order parameters. J. Phys. C: Solid State Phys. 1975, 8, 567-577.

(C) 2012 by the authors; licensee MDPI, Basel, Switzerland. This article is an open access article distributed under the terms and conditions of the Creative Commons Attribution license (http://creativecommons.org/licenses/by/3.0/). 